\title{
Performance of the ATLAS detector using first collision data
}

\section{The ATLAS collaboration}

ABSTRACT: More than half a million minimum-bias events of LHC collision data were collected by the ATLAS experiment in December 2009 at centre-of-mass energies of $0.9 \mathrm{TeV}$ and $2.36 \mathrm{TeV}$. This paper reports on studies of the initial performance of the ATLAS detector from these data. Comparisons between data and Monte Carlo predictions are shown for distributions of several track- and calorimeter-based quantities. The good performance of the ATLAS detector in these first data gives confidence for successful running at higher energies.

KEYwORDS: Hadron-Hadron Scattering

ARXIV EPRINT: 1005.5254 


\section{Contents}

1 Introduction 1

2 The ATLAS detector $\quad 1$

3 Data-taking performance and event selection 2

3.1 Trigger/DAQ system 3

3.2 Event selection 4

3.3 Luminosity measurement 5

4 Monte Carlo simulation $\quad 6$

$\begin{array}{lll}5 & \text { Tracking performance } & 6\end{array}$

5.1 Hits on tracks 6

$5.2 K_{\mathrm{S}}^{0}$ studies $\quad 9$

$\begin{array}{lll}5.3 \mathrm{~d} E / \mathrm{d} x \text { and } \phi(1020) \text { identification } & 10\end{array}$

$\begin{array}{lll}5.4 & \text { Secondary vertex tagging } & 12\end{array}$

$\begin{array}{ll}\text { 5.5 Particle identification using transition radiation } & 15\end{array}$

$\begin{array}{lll}5.6 & \text { Tracking efficiency for Level-2 trigger } & 16\end{array}$

$\begin{array}{lll}6 & \text { Electrons and photons } & \mathbf{1 7}\end{array}$

$\begin{array}{lll}6.1 & \text { Electron and photon reconstruction } & 17\end{array}$

$\begin{array}{ll}6.2 & \text { Electron and photon identification } \\ & 18\end{array}$

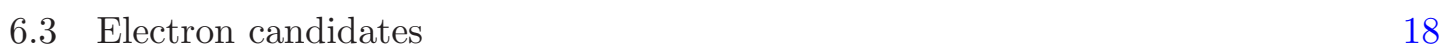

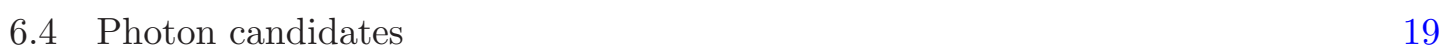

6.5 First-level electron and photon trigger performance 20

6.6 Electron and photon identification variables 20

$\begin{array}{lll}\text { 6.6.1 Calorimeter variables } & 20\end{array}$

6.6.2 Tracking and track-cluster matching variables 21

6.6.3 Use of the TRT for electron identification 25

$\begin{array}{lll}6.7 & \text { Photon conversions } & 25\end{array}$

6.8 Reconstruction of $\pi^{0}$ and $\eta$ mesons 28

6.8.1 Extraction of $\pi^{0} \rightarrow \gamma \gamma$ signal 28

$\begin{array}{lll}6.8 .2 & \pi^{0} \text { mass fit } & 29\end{array}$

$\begin{array}{ll}\text { 6.8.3 Extraction of the } \eta \rightarrow \gamma \gamma \text { signal } & 30\end{array}$

$\begin{array}{lll}7 & \text { Jets } & 31\end{array}$

$\begin{array}{lll}7.1 & \text { Jets from calorimeter clusters } & 31\end{array}$

$\begin{array}{lll}7.2 & \text { Performance of the first level jet trigger } & 32\end{array}$

$\begin{array}{lll}7.3 & \text { Tau studies using jets } & 33\end{array}$

8 Missing transverse energy $\quad 34$ 


\section{Introduction}

In December 2009, the ATLAS detector [1] recorded data from a first series of LHC runs at centre-of-mass energies of $0.9 \mathrm{TeV}$ and $2.36 \mathrm{TeV}$. When the beams were colliding and declared to be stable by the LHC operators, all main detector components were fully operational and all levels of the trigger and data acquisition system performed as expected, assuring smooth and well monitored data taking. The data sample at $0.9 \mathrm{TeV}$ contains nearly 400000 events recorded with high-quality calorimeter and tracking information, corresponding to an integrated luminosity of approximately $9 \mu \mathrm{b}^{-1}[2]$. The data at $2.36 \mathrm{TeV}$, 36000 events, which are only used here for calorimeter studies, correspond to approximately $0.7 \mu \mathrm{b}^{-1}$. These data sets do not contain very many high- $p_{\mathrm{T}}$ objects, and therefore do not correspond to the environment for which ATLAS was designed.

The ATLAS detector was thoroughly commissioned and initial calibration and performance studies were done using cosmic ray data recorded during 2008 and 2009. Performance close to design goals was obtained for the different detector components, details can be found in refs. [3-6].

This paper presents performance established with data taken in first collisions in 2009. The detector components are outlined in section 2. The trigger and data acquisition performance together with the initial event selection are discussed in section 3 ; the simulation to which the data are compared is explained in section 4 . The performance of the inner tracking system is reviewed in section 5 , and the combined analysis of calorimeter data and tracking information to study electrons and photons is discussed in section 6. Studies of jets and missing transverse energy, $E_{\mathrm{T}}^{\text {miss }}$, using the calorimeter cells are presented in sections 7 and 8. Finally, kinematic distributions of the first reconstructed muon candidates are shown in section 9 .

\section{The ATLAS detector}

The ATLAS detector [1] covers almost the entire solid angle around the nominal interaction point and comprises the following sub-components:

- An inner tracking system: operating inside an axial magnetic field of $2 \mathrm{~T}$, it is based on three types of tracking devices. These are an outer tracker using straw tubes with particle identification capabilities based on transition radiation (Transition Radiation Tracker, TRT), a silicon strip detector (SemiConductor Tracker, SCT) and an innermost silicon pixel detector (Pixel). 


\begin{tabular}{|lcccccccc|}
\hline & Pixel & SCT & TRT & LAr & Tile & MDT & RPC & TGC \\
\hline Efficiency [\%] & 80.9 & 86.2 & 100 & 99.0 & 100 & 87.4 & 88.6 & 84.4 \\
\hline
\end{tabular}

Table 1. Luminosity-weighted fraction of the time during stable beam operation for which the different detectors were able to take data under nominal conditions.

- A hybrid calorimeter system: for the electromagnetic portion (EM), the hadronic end-cap (HEC) and the forward calorimeter (FCal) a liquid argon (LAr) technology with different types of absorber materials is used. The central hadronic calorimeter (Tile) is a sampling calorimeter with steel as the absorber material and scintillator as the active medium. The electromagnetic sections use an accordion geometry to ensure fast and uniform response. A presampler detector, to correct for energy losses in the upstream material, is installed in front of the EM calorimeter in the range $|\eta|<1.8 .^{1}$

- A large muon spectrometer: an air-core toroid system generates an average field of $0.5 \mathrm{~T}(1 \mathrm{~T})$, in the barrel (end-cap) region of this spectrometer, resulting in a bending power between 2.0 and 7.5 Tm. Over most of the $\eta$-range, tracks are measured by Monitored Drift Tubes (MDT); in the high $\eta$-regime the closest of four wheels to the interaction region is instrumented with Cathode Strip Chambers (CSC). Trigger information is provided by Thin Gap Chambers (TGC) in the end-cap and Resistive Plate Chambers (RPC) in the barrel.

- Specialized detectors in the forward region: two dedicated forward detectors, the LUCID Cherenkov counter and the Zero Degree Calorimeter (ZDC). In addition the BPTX, an electrostatic beam-pickup which monitors the timing of the beam near ATLAS and two scintillator wheels (MBTS) were mounted in front of the electromagnetic end-caps to provide trigger signals with minimum bias.

\section{Data-taking performance and event selection}

The ATLAS operating procedure in 2009 maintained the calorimeters and TRT in standard operating conditions, but the silicon trackers and muon chambers were at a reduced or 'standby' voltage until after stable beams were declared by the LHC. Most of the studies in this paper required the tracking detectors to be in operating conditions and used approximately 400000 events while the $E_{\mathrm{T}}^{\text {miss }}$ studies only required the calorimeters and used some 600000 and 36000 events at $0.9 \mathrm{TeV}$ and $2.36 \mathrm{TeV}$ respectively. The luminosity-weighted availability of the various sub-detectors (see section 3.3) during stable beam operations is summarized in table 1.

\footnotetext{
${ }^{1}$ The origin of the coordinate system used to describe the ATLAS detector is the nominal interaction point. The positive $x$ axis is defined as pointing from the interaction point to the centre of the LHC ring, the positive $y$ axis is defined as pointing upwards and the beam direction defines the $z$ axis of a righthanded coordinate system. Transverse momenta are measured in the $x-y$ plane with radius $r$. Polar $(\theta)$ and azimuthal $(\phi)$ angles are measured with respect to this reference system. The pseudorapidity is defined as $\eta=-\ln \tan (\theta / 2)$.
} 

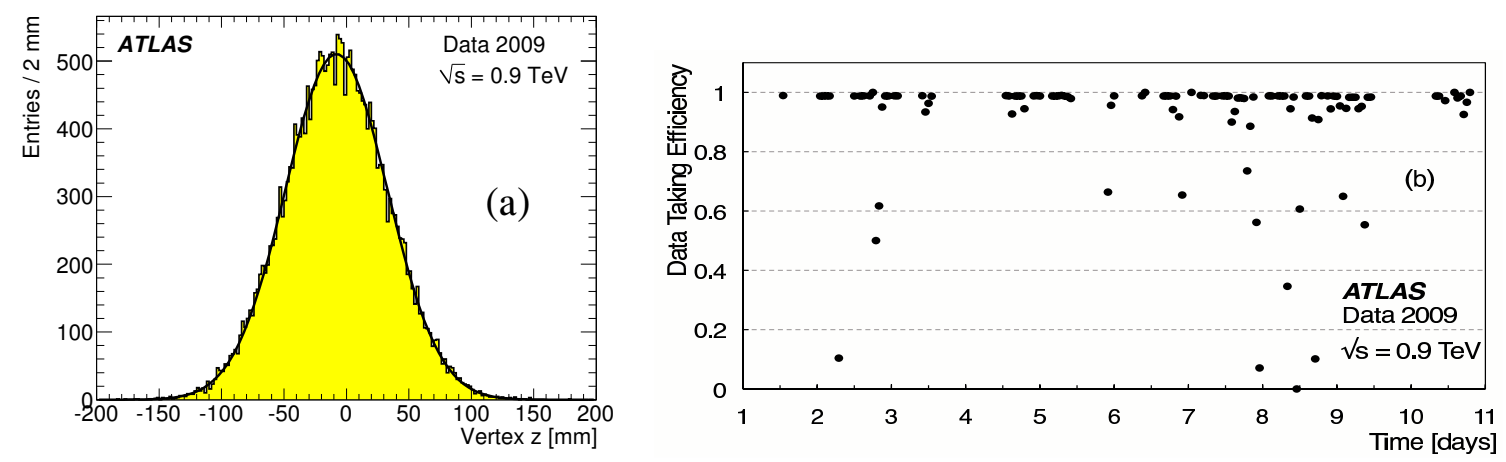

Figure 1. (a) Reconstructed $z$-vertex distribution calculated online by the higher level trigger for monitoring purposes. The width includes a small contribution from the experimental resolution. (b) Data-taking efficiency for periods with two circulating beams in December 2009.

\subsection{Trigger/DAQ system}

The ATLAS trigger and data acquisition (TDAQ) is a multi-level system with buffering at all levels [1]. Trigger decisions are based on calculations done at three consecutive trigger levels. While decisions at the first two levels are pending, the data acquisition system buffers the event data from each sub-detector. Complete events are built after the second level decision. The first level trigger (L1) is largely based on custom built electronics. It incorporates timing from the BPTX and coarse detector information from the muon trigger chambers and the trigger towers of the calorimeters, along with multiplicity information from the MBTS scintillators and the ATLAS forward detectors, LUCID and ZDC. The L1 system is designed to select events at a rate not exceeding $75 \mathrm{kHz}$ from an input rate of 40 $\mathrm{MHz}$ and identify regions-of-interest (RoIs), needed by the high-level trigger system (HLT), for potentially interesting physics objects. HLT runs on a processor farm and comprises the second level (L2) and the third level trigger or Event Filter (EF). The L2 system evaluates event characteristics by examining the RoIs using more detector information and more complete algorithms. The EF analyzes the L2 selected events again looking at the RoIs' measurements. The analysis of the complete event is also possible. The output rate is reduced to approximately $200 \mathrm{~Hz}$.

All trigger decisions used were made by the L1 systems as the luminosity was low. Minimum-bias events were triggered by a coincidence between the the BPTX and signals indicating hits in one or both of the MBTS scintillator wheels. However, the functionality of the main L2 and EF algorithms, for example those used for inner track reconstruction and jet finding, were validated by running in 'passthrough' mode, i.e. calculating relevant L2 and EF decisions without rejecting events. Important distributions like the vertex position (figure 1(a)) were monitored online and different data streams for fast analysis feedback based on L1 trigger decisions were provided. For the December 2009 running period the data-taking efficiency of the overall Trigger/DAQ system, as illustrated in figure 1(b), averaged to $90 \%$. 

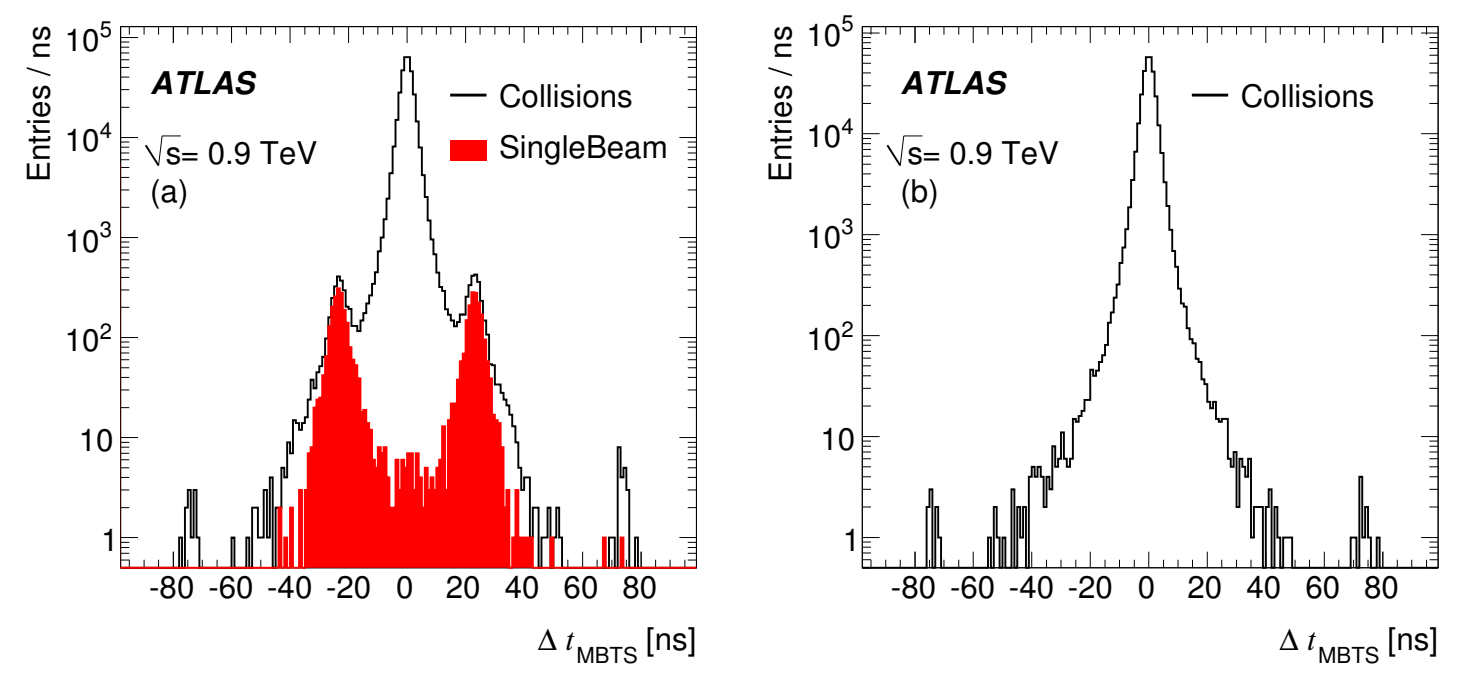

Figure 2. Time difference $\Delta t_{\mathrm{M} B T S}$ of hits recorded by the two MBTS scintillator wheels mounted in front of the electromagnetic end-cap wheels on both sides of the ATLAS detector; (a) time difference without any selection and (b) requiring a well-reconstructed vertex.

\subsection{Event selection}

To select collision candidates and remove beam-related background two different strategies were employed:

- For those studies based mainly on track information, the presence of a primary vertex, reconstructed using at least three tracks with sufficient transverse momenta, typically $p_{T}>150 \mathrm{MeV}$, and a transverse distance of closest approach compatible with the nominal interaction point are required. This selection strategy, which was used in ref. [2], uses events triggered by a single hit in one of the two MBTS scintillator wheels.

- Alternatively, the selection is based on the timing difference of signals detected on both sides of the ATLAS detector. Coincident signals, within a time window of 5 or 10 ns from either the electromagnetic calorimeters (end-cap or FCal) or from the two MBTS wheels, respectively, are required. The event must again be triggered by an MBTS signal. In case no timing coincidence is found, a two hit MBTS trigger with at least one hit per side is required.

The detailed track quality criteria used for the first strategy vary slightly for the different studies presented and are described later when appropriate.

Without any event selection the MBTS-triggered events contain backgrounds from beam-related events as shown in figure $2(\mathrm{a})$, where the time difference, $\Delta t_{\mathrm{MBTS}}$, of MBTS signals recorded on both sides of the ATLAS detector is depicted. For events coming from the interaction point $\Delta t_{\mathrm{MBTS}}$ is small. Beam-related background produced upstream or downstream should have $\Delta t_{\text {MBTS }}$ around $25 \mathrm{~ns}$, with the sign giving the direction. Eighty percent of single beam events are missing timing information on one or both sides 


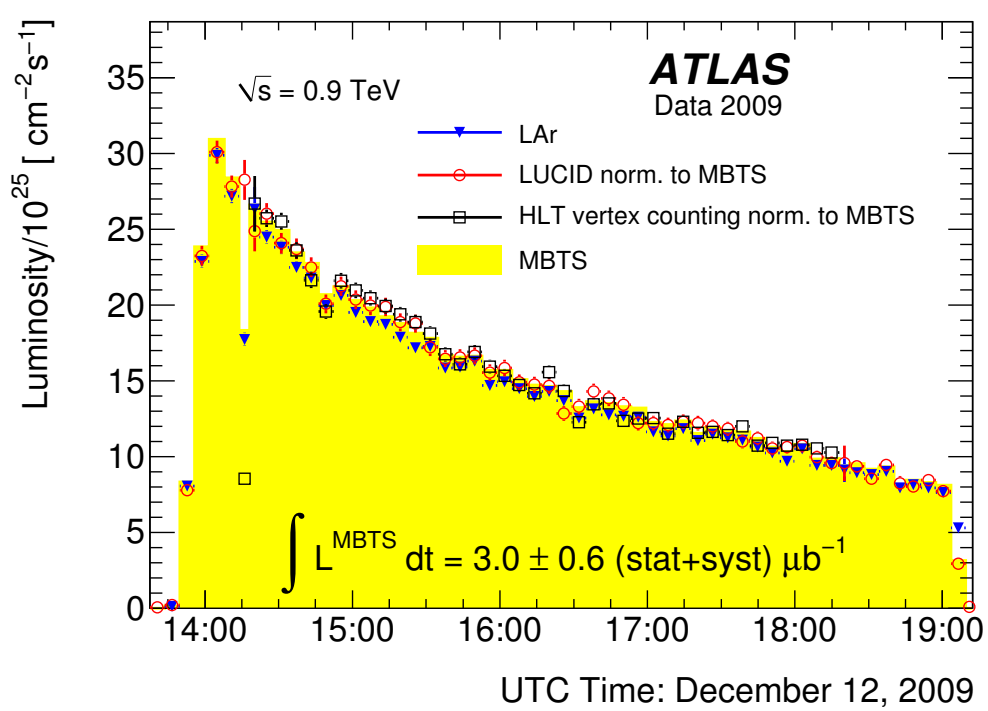

Figure 3. Instantaneous luminosity measured by the MBTS and LAr, with superimposed the LUCID and HLT vertex counting estimates normalized in such a way to give the same integrated luminosity as measured with the MBTS system. All measurements are corrected for TDAQ deadtime, except LUCID which is free from dead time effects. The short luminosity drop at 14:15 is due to inhibiting the trigger for ramping up the silicon detectors after declaration of stable LHC beams.

and are therefore not shown. Requiring a well-reconstructed vertex with track quality requirements reduces the beam-related background by more than three orders of magnitude while retaining genuine collision events (figure $2(b)$ ). There are twelve single-beam events which meet this vertex requirement, but all of them are missing timing information and are not shown.

\subsection{Luminosity measurement}

The luminosity during the 2009 ATLAS data-taking period was estimated offline based upon the timing distributions measured by the MBTS. Events with signals detected on opposite ends of the ATLAS detector in the MBTS are counted. After background subtraction the luminosity is calculated using the number of events with a timing difference consistent with particles originating from the interaction point (see figure 2), the expected minimum-bias cross section and the event selection efficiency determined from data and Monte Carlo. The MBTS detector is used for the absolute luminosity determination because of its high trigger efficiency for non-diffractive events [2]. The uncertainty on the luminosity, dominated by the understanding of the modelling of inelastic pp interactions, is estimated to be around $20 \%$.

Figure 3 shows the luminosity as a function of time, as measured using the MBTS system as well as with three other techniques: timing in the LAr, the LUCID relative luminosity monitor and particle vertices reconstructed online. The LAr technique has a slightly larger systematic uncertainty in the accepted cross section than that from the MBTS and produces a result which agrees to $4 \%$. The other methods are normalized to the MBTS measurement. 


\section{Monte Carlo simulation}

Monte Carlo samples produced with the PYTHIA 6.4.21 [7] event generator are used for comparison with the data. ATLAS selected an optimized parameter set [8], using the $p_{\mathrm{T}^{-}}$ ordered parton shower, tuned to describe the underlying event and minimum bias data from Tevatron measurements at $0.63 \mathrm{TeV}$ and $1.8 \mathrm{TeV}$. The parton content of the proton is parameterized by the MRST LO* parton distribution functions [9].

Various samples of Monte Carlo events were generated for single-diffractive, doublediffractive and non-diffractive processes in pp collisions. The different contributions in the generated samples were mixed according to the cross-sections calculated by the generator. There was no contribution from cosmic ray events in this simulation. All the events were processed through the ATLAS detector simulation program [10], which is based on GEANT4 [11]. This simulation software has also been systematically compared to testbeam data over the past decade (see e.g. ref. [12]) and it was constantly improved to describe these data. After the detector simulation the events were reconstructed and analyzed by the same software chain also used for data.

The beam position and size, which did not correspond precisely to those used in the simulation prepared beforehand, have a significant impact on some distributions, particularly for detectors close to the interaction region. The length of the luminous region, as seen in figure 1(a), is approximately half that expected, and the simulated events were re-weighted to match this. The transverse offset in the simulation of about $2 \mathrm{~mm}$ cannot be corrected for by this method.

The distributions presented in this paper always show the simulated sample normalized to the number of data events in the figure.

\section{Tracking performance}

The inner tracking system measures charged particle tracks at all $\phi$ and with pseudorapidity $|\eta|<2.5$. The pixel detector is closest to the beam, covering radial distances of 50-150 mm with three layers both in the barrel region and in each end-cap. The innermost Pixel layer (known as the B-layer) is located just outside the beam pipe at a radius of $50 \mathrm{~mm}$. The pixels are followed, at radii between $299-560 \mathrm{~mm}$, by the silicon strip detector known as the SCT. This provides 4 (barrel) or 9 (end-cap) double layers of detectors. The Pixels are followed, for radii between $563-1066 \mathrm{~mm}$, by the TRT. The TRT straw layout is designed so that charged particles with transverse momentum $p_{\mathrm{T}}>0.5 \mathrm{GeV}$ and with pseudorapidity $|\eta|<2.0$ cross typically more than 30 straws. The intrinsic position resolutions in $r \phi$ for the Pixels, the SCT and the TRT are 10, 17 and $130 \mu \mathrm{m}$, respectively. For the Pixels and the SCT the other space coordinate is measured with 115 and $580 \mu \mathrm{m}$ accuracy, where the $\mathrm{SCT}$ measurement derives from a $40 \mathrm{mrad}$ stereo angle between the two wafers in a layer.

\subsection{Hits on tracks}

The sample of minimum-bias events provides approximately two million charged particles with $p_{\mathrm{T}}$ over $500 \mathrm{MeV}$ through the central detectors of ATLAS. Their trajectories in the 

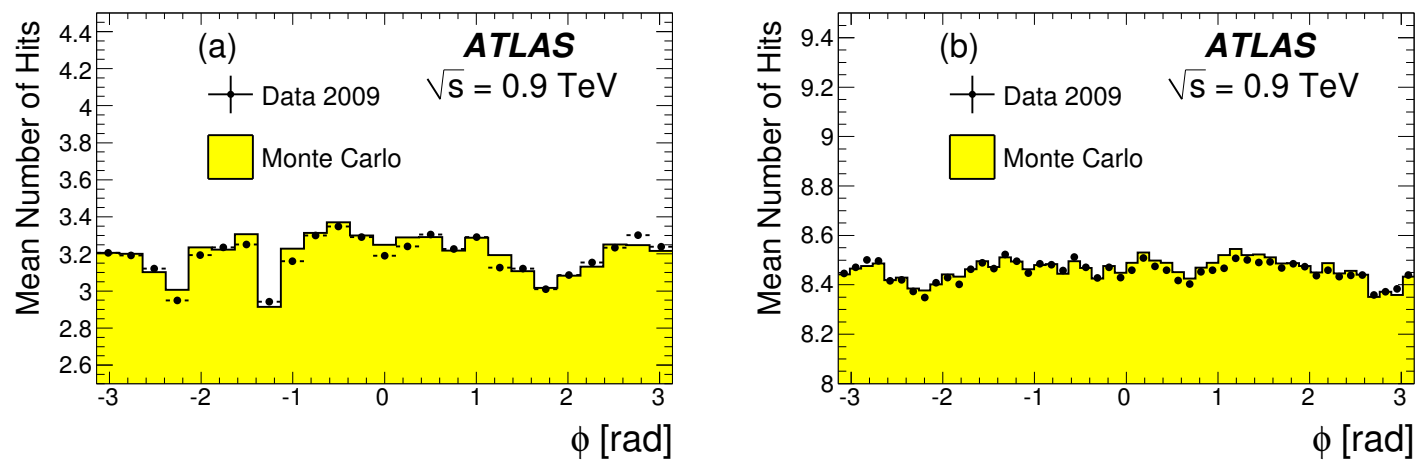

Figure 4. A comparison of data and simulation in the average number of hits in (a) the Pixels and (b) the SCT versus $\phi$ on selected tracks. Comparable distributions versus $\eta$ can be found in ref. [2].

inner detector were reconstructed using a pattern recognition algorithm that starts with the silicon information and adds TRT hits. This 'inside-out' tracking procedure selects track candidates with transverse momenta above $500 \mathrm{MeV}$ [13]. Two further pattern recognition steps were run, each looking only at hits not previously used: one starts from the TRT and works inwards adding silicon hits as it progresses and the other repeats the first step, but with parameters adjusted to allow particle transverse momenta down to $100 \mathrm{MeV}$. The multiple algorithms are necessary partly because a $100 \mathrm{MeV} p_{\mathrm{T}}$ charged particle has a radius of curvature of about $17 \mathrm{~cm}$ in the ATLAS magnetic field and will not reach the TRT.

The track selection requirements vary slightly among the analyses presented here. A typical set of selections is that charged particle tracks are required to have $p_{\mathrm{T}}>0.5 \mathrm{GeV}$, $\geq 1$ Pixel hit, $\geq 6 \mathrm{SCT}$ hits and impact parameters with respect to the primary vertex of $\left|d_{0}\right|<1.5 \mathrm{~mm}$ and $\left|z_{0} \sin \theta\right|<1.5 \mathrm{~mm}$. The transverse impact parameter, $d_{0}$, of a track is its distance from the primary vertex at the point of closest approach when projecting into the transverse plane, signed negative if the extrapolation inwards has the primary vertex to the right, $z_{0}$ is the longitudinal distance at that point.

The hit distributions in the silicon detectors as a function of $\phi$ are shown in figure 4 for tracks passing these requirements in data and simulation. The fluctuations seen in $\phi$ correspond to non-responsive detector modules which are modelled in the simulation. A small mis-match between data and simulation arises because the simulated beam had a transverse displacement of about $2 \mathrm{~mm}$ from the true position, as discussed in section 4 .

The efficiency of the individual TRT straws is displayed in figure 5 as a function of the distance of the test track to the wire in the centre of the straw. The efficiency for data and simulation, barrel and end-cap, has a plateau close to $94 \%$.

The alignment of the tracking detectors benefited from the precision construction and survey followed by an extended period of data taking using cosmic ray muons [5]. The alignment was improved using the $0.9 \mathrm{TeV}$ collision data, although the particles have rather low momentum and therefore their tracks suffer from multiple scattering. The quality of the alignment can be checked by the study of the residuals, which are defined as the measured hit position minus that expected from the track extrapolation. 

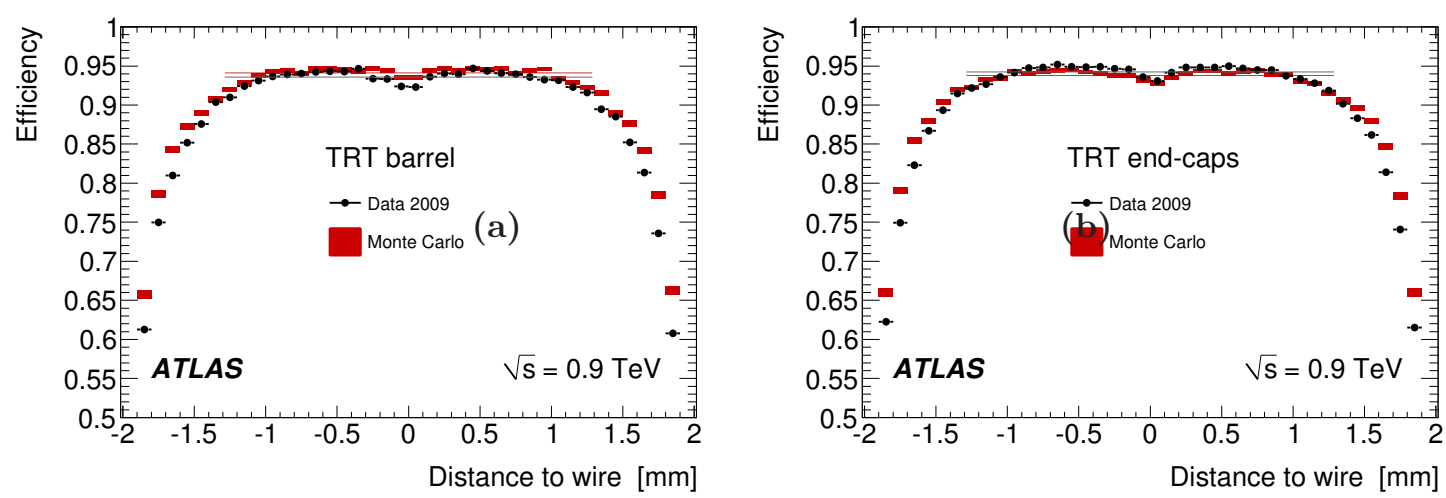

Figure 5. TRT hit efficiency as a function of the distance of the track from the wire in the centre of the straw in (a) the barrel and (b) the end-caps.
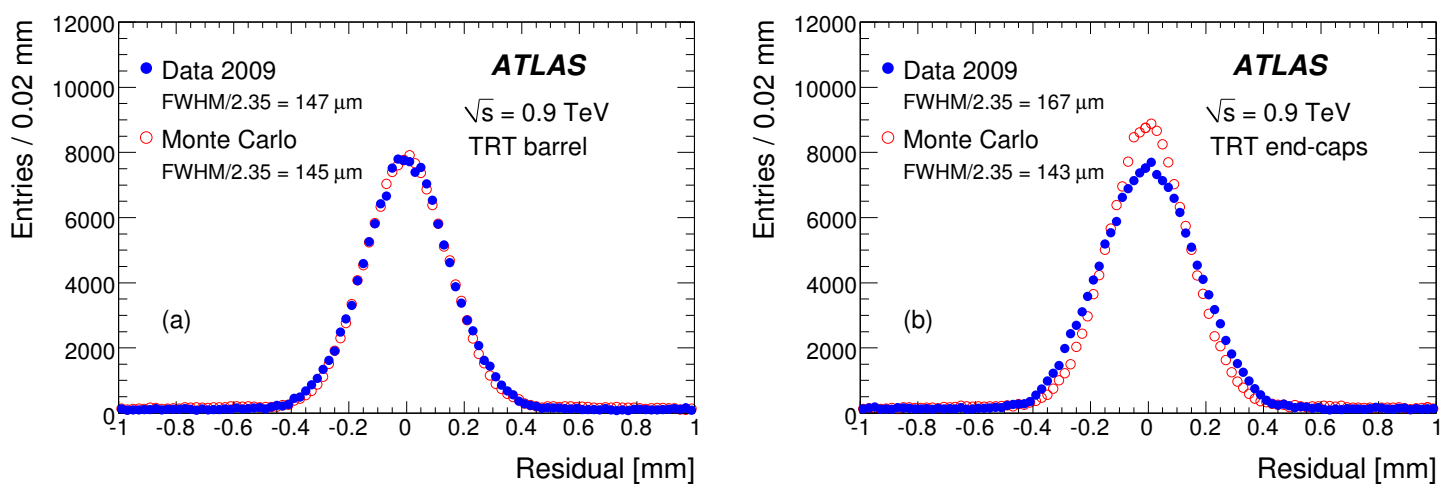

Figure 6. Unbiased residual distributions in the TRT barrel (a) and end-caps (b). The data points are in filled circles and the simulation in empty ones.

Unbiased residuals between tracks and barrel TRT hits are plotted in figure 6. This figure is made using charged particles with $p_{\mathrm{T}}>1 \mathrm{GeV}$ with over 6 hits in the SCT and at least 14 in the TRT. The equivalent Gaussian width is extracted from the full-width at half maximum. The end-cap shows a resolution somewhat worse than simulation, while in the barrel part of the detector, where a higher cosmic ray flux can be used for alignment, data and simulation are in close agreement.

Unbiased $x$ residuals from the silicon detectors are shown in figure 7 , where $x$ refers to the more precise local coordinate on the detector. Charged particles are selected to have $p_{\mathrm{T}}>2 \mathrm{GeV}$. The equivalent Gaussian width is extracted from the full-width at half maximum. The width of the resulting distributions in data are within about $15 \%$ of those found in a simulation with no alignment errors, showing that the remaining impact on the residual widths from imperfect alignment in data is at the level of approximately 10-15 $\mu \mathrm{m}$ for the pixels and of $20 \mu \mathrm{m}$ for the SCT. 

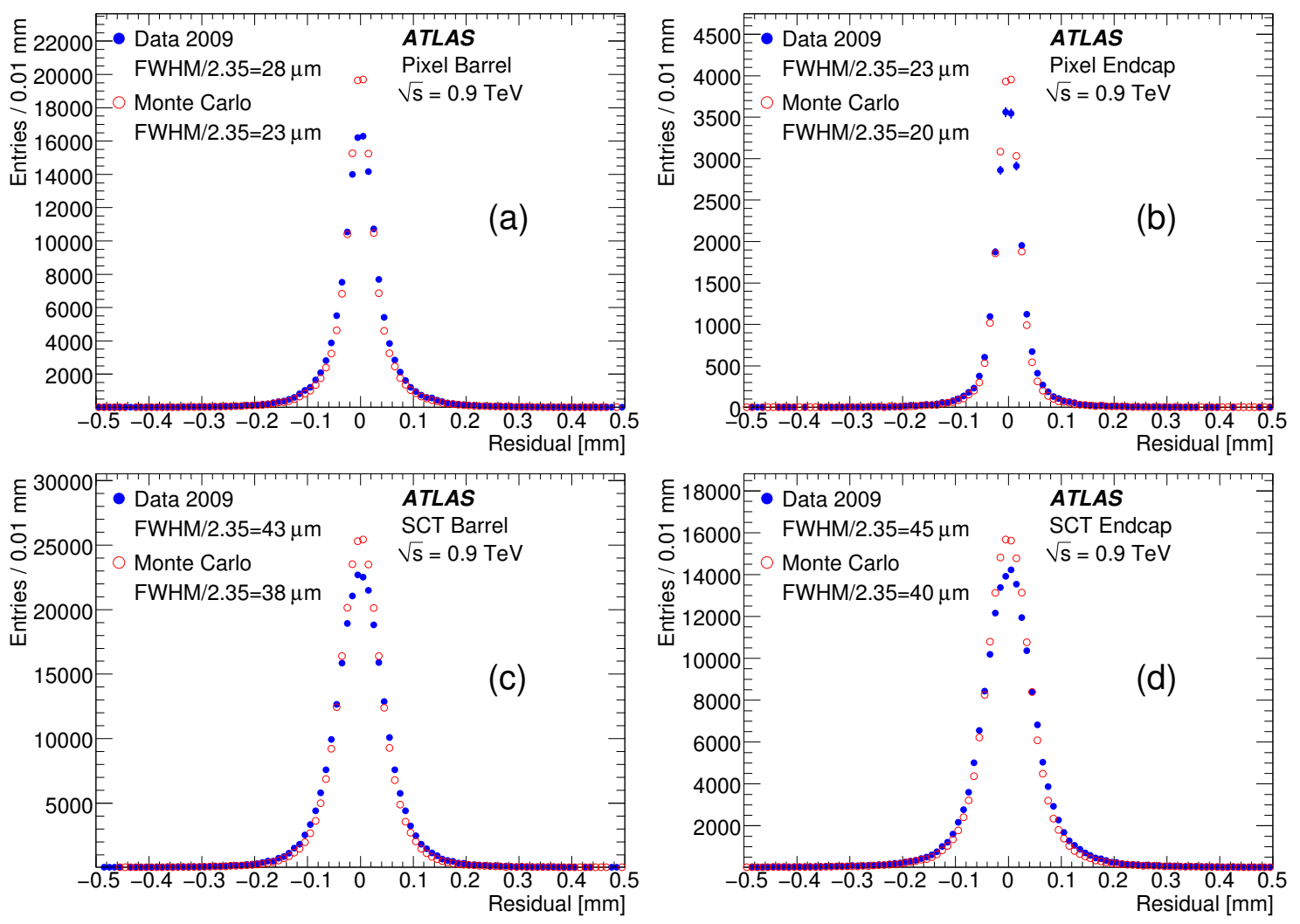

Figure 7. The distributions of the silicon detector unbiased residuals for (a) the pixel barrel, (b) the pixel end-cap, (c) the SCT barrel, (d) the SCT end-cap. The data are in solid circles, the simulation, which has a perfect alignment, is shown with open ones.

\section{$5.2 \quad K_{\mathrm{S}}^{0}$ studies}

The momentum scale and resolution of the tracker, and energy loss with in, were all investigated by studying the $K_{\mathrm{S}}^{0}$ to $\pi^{+} \pi^{-}$decay. The reconstruction requires pairs of oppositely-charged particles compatible with coming from a common vertex. This vertex, in the transverse plane, must be more than $0.2 \mathrm{~mm}$ from the primary vertex. The cosine of the angle between the flight path relative to the primary vertex and the momentum vector of the candidate, $\cos \theta_{K}$, is required to exceed 0.8 . The invariant mass distribution, calculated assuming that both charged particles are pions is shown in figure 8 . The simulated signal and background are separately normalized to the data, and the position and width of the $K_{\mathrm{S}}^{0}$ mass peak are fitted using a Gaussian. The peak in data is at $m_{\pi \pi}=497.5 \pm 0.1 \mathrm{MeV}$, in agreement with the PDG average [14].

In order to test the momentum scale and resolution of the detector the reconstructed pions in the simulation are adjusted by parameters $\mu_{\mathrm{tr}}$, which scales the $1 / p_{\mathrm{T}}$, and $\sigma_{\mathrm{tr}}$, a Gaussian smearing on $\mu_{\mathrm{tr}}$. The values of these parameters which best fit the observed $K_{\mathrm{S}}^{0}$ mass and width in the barrel region are $\mu_{\mathrm{tr}}=1.0004 \pm 0.0002$ and $\sigma_{\mathrm{tr}}=0.0040 \pm 0.0015$. Thus the momentum scale for these barrel charged particles is known at better than the one per mille level, which is as expected from the accuracy of the solenoid magnet field- 


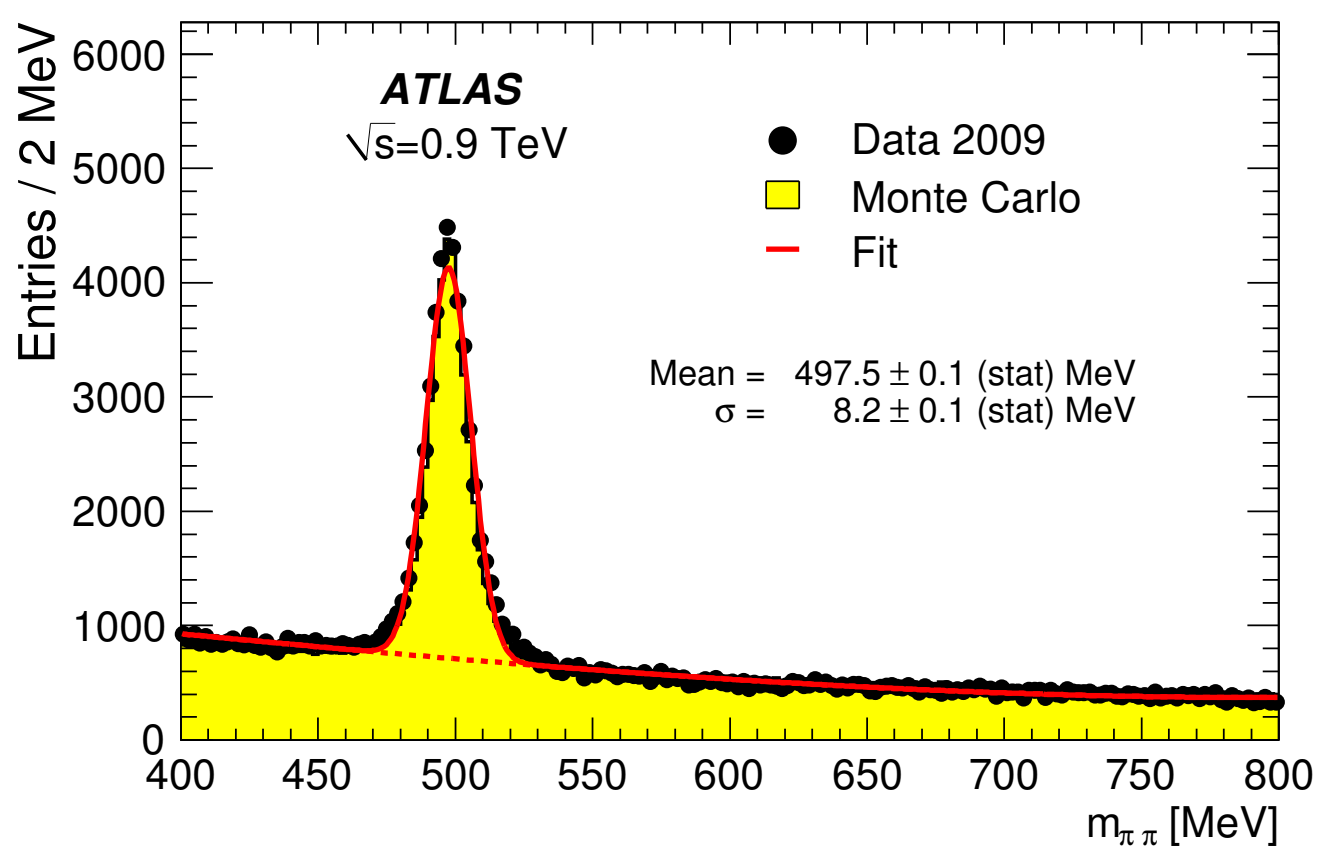

Figure 8. The $K_{\mathrm{S}}^{0}$ candidate mass distribution using impact parameter and lifetime selections. The simulated signal and background are separately normalized to the data.

mapping performed before installation of the inner detector [15]. This, and subsequent $K_{\mathrm{S}}^{0}$ studies, use a tighter cut of 0.99 on $\cos \theta_{K}$.

In the end-cap regions there is evidence for a degraded resolution, especially at low momentum. Charged particles with $p_{T}$ below $500 \mathrm{MeV}$ require a $\sigma_{\text {tr }}$ of $0.024 \pm 0.004$ and $0.022 \pm 0.004$ in the negative and positive end-caps, respectively, to match the data, suggesting some material is missing in the description of the end-caps. The momentum scale in the end-caps is compatible with the nominal within errors of 1 to 2 per mille.

The $K_{\mathrm{S}}^{0}$ peak was also used to investigate the amount of material in the inner tracker as a function of radius. The mass reconstructed in data, divided by that found in simulation, is shown in figure 9 as a function of decay radius.

Deviations of this ratio from unity would expose differences between the real detector and the model used for simulation. The results for special simulation samples with approximately $10 \%$ and $20 \%$ fractional increase in the radiation length of the silicon systems included by increasing the density of some of the support structures are also shown in figure 9. These results suggest that discrepancies of material between the data and the simulation must be significantly smaller than $10 \%$ of the material thickness in the inner silicon barrels.

\section{$5.3 \mathrm{~d} E / \mathbf{d} x$ and $\phi(1020)$ identification}

One feature of the Pixel tracking system is a time-over-threshold measurement for the signal which was used to extract the specific energy loss $\mathrm{d} E / \mathrm{d} x$. Tracks with more than one Pixel hit were studied and the mean $\mathrm{d} E / \mathrm{d} x$ was found for each after the highest was 


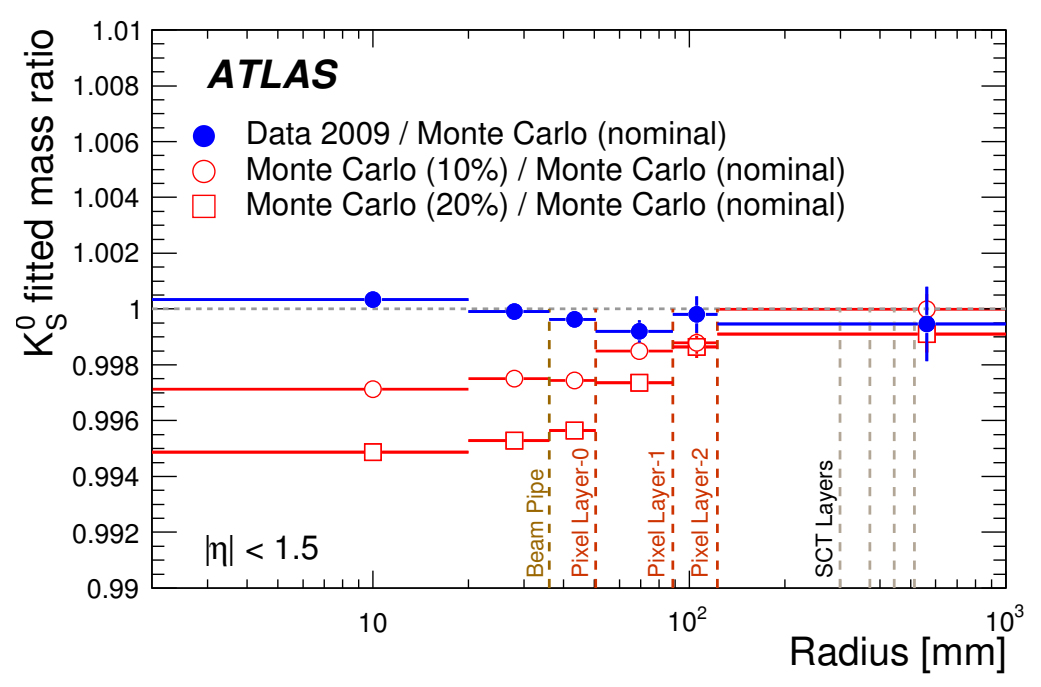

Figure 9. The fitted $K_{\mathrm{S}}^{0}$ mass divided by the value found in nominal MC simulation as a function of the reconstructed decay position. The filled circles show the data, and the open symbols are for simulation samples with approximately $10 \%$ and $20 \%$ more silicon tracker material added. The horizontal dotted line is to guide the eye.

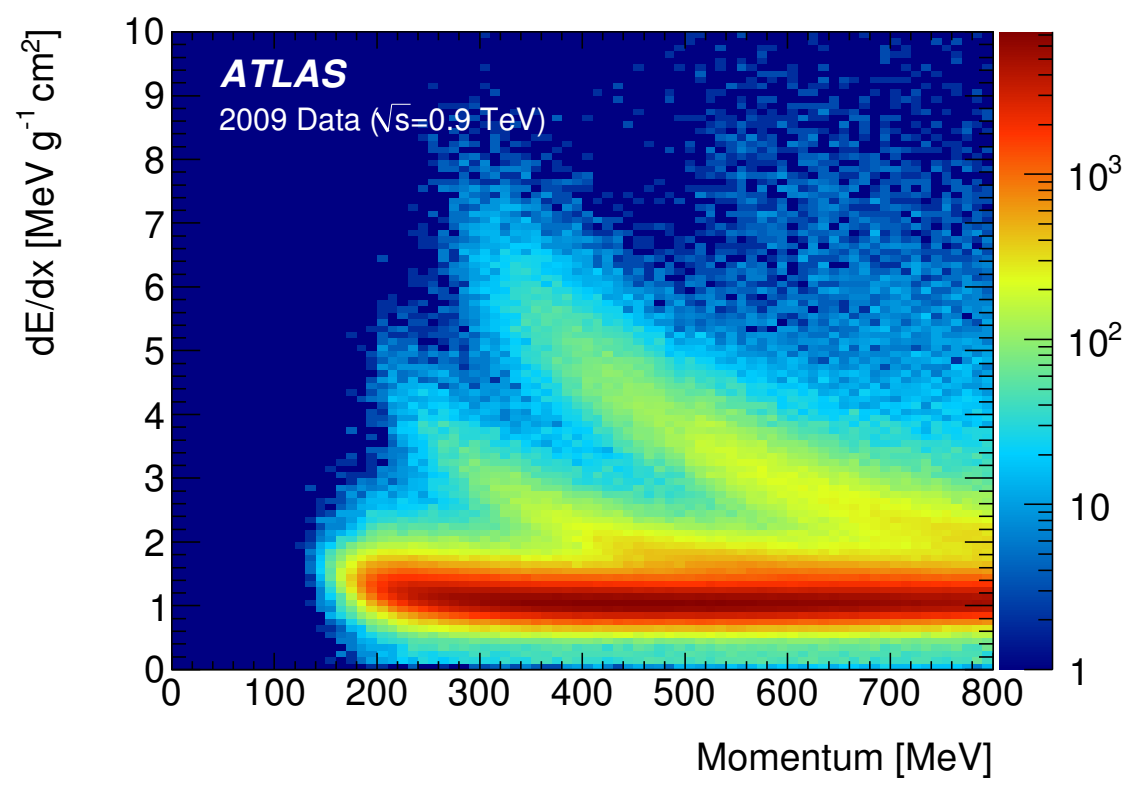

Figure 10. The $\mathrm{d} E / \mathrm{d} x$ measured in data as a function of momentum.

removed to reduce the effect of Landau fluctuations. Figure 10 shows the distribution observed in the data. Bands corresponding to different particle species are clearly visible.

In the observation of $\phi \rightarrow K^{+} K^{-}$identification of the $K^{ \pm}$reduces the combinatorial background. The identification of kaon candidates through $\mathrm{d} E / \mathrm{d} x$ proceeds by finding the probability density functions of pions $\left(p_{\text {pion }}\right)$, kaons $\left(p_{\text {kaon }}\right)$ and protons $\left(p_{\text {proton }}\right)$ in the simulation as a function of momentum and $\mathrm{d} E / \mathrm{d} x$. This is done via fitting the observed 


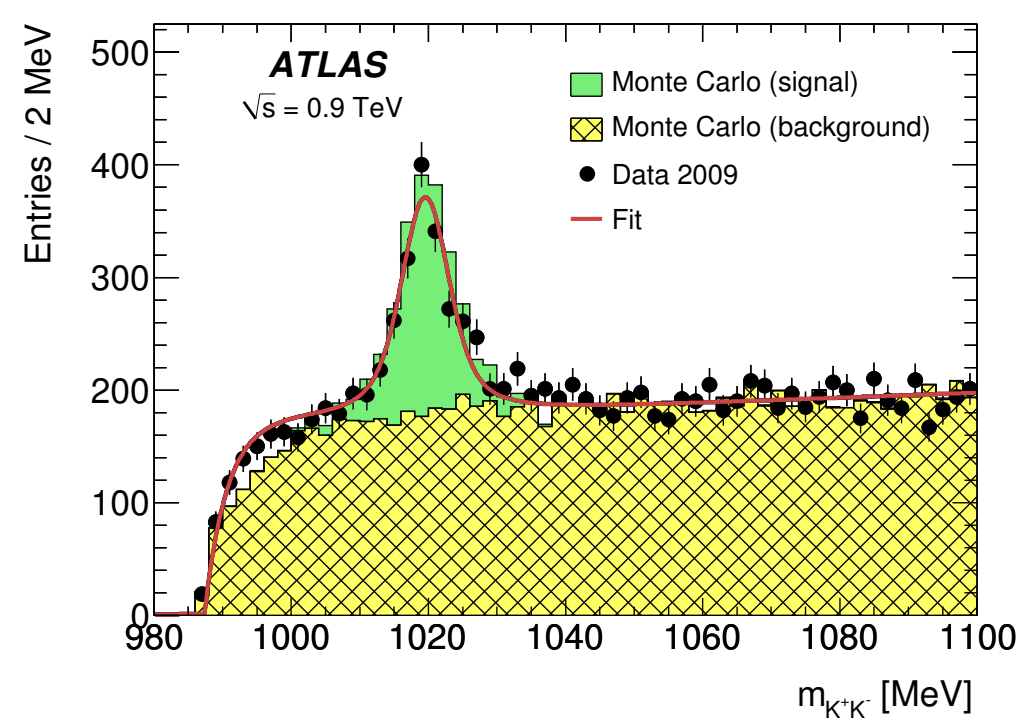

Figure 11. The measured and simulated mass spectra of $K^{+} K^{-}$pairs. The $\phi$ peak is fitted with a Breit-Wigner with a fixed width convoluted with a Gaussian. Both kaons must be identified through the $\mathrm{d} E / \mathrm{d} x$ measurement.

value in simulation using a Gaussian function whose parameters are momentum dependent. The simulation models the data with an accuracy of about $10 \%$.

The tracks used in the reconstruction of the $\phi$ meson must have more than one hit in the Pixel system and an impact parameter within $3 \sigma$ of the primary vertex. The track fit was re-run using the kaon mass hypothesis for the energy loss. The simulation shows that after re-fitting the kaon momenta are underestimated by up to $10 \mathrm{MeV}$ and a corresponding correction is applied. This changes the reconstructed $\phi$ mass by approximately $0.3 \mathrm{MeV}$. All oppositely charged particle pairs where both momenta, reconstructed under the kaon hypothesis, are below $800 \mathrm{MeV}$ are considered.

Figure 11 shows the resulting mass distribution for the $K^{+} K^{-}$candidate pairs, selected using charged particles with $200<p_{\mathrm{T}}<800 \mathrm{MeV}$ and a kaon $\mathrm{d} E / \mathrm{d} x$ tag. The selection cuts were chosen to yield optimal signal significance on simulated events; a measure which was greatly improved using the $\mathrm{d} E / \mathrm{d} x$ information.

The background and signal levels in the simulation were scaled independently to match the data. The fit allowed the mass and experimental resolution to vary, while keeping the natural width fixed to the PDG [14] average. The mass was found to be $1019.5 \pm 0.3 \mathrm{MeV}$, in agreement with the expected value. The fitted experimental resolution in data is $2.5 \pm 0.5 \mathrm{MeV}$ and matches the $2.4 \pm 0.3 \mathrm{MeV}$ found in Monte Carlo simulation.

\subsection{Secondary vertex tagging}

An important role of the tracking system is the identification of heavy flavour hadrons. There are several tagging algorithms developed in ATLAS. Some performance figures for two algorithms, the impact parameter and the secondary vertex tagging algorithm, are presented in the following. 

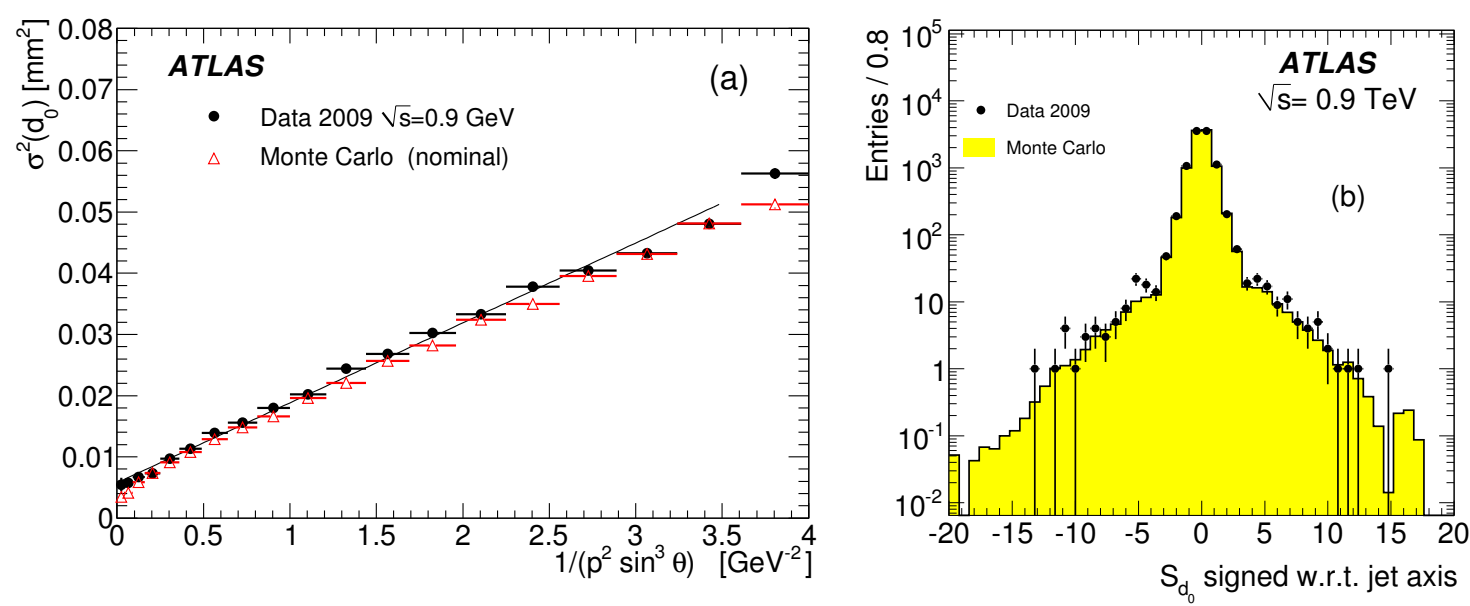

Figure 12. (a) The variance of the $d_{0}$ distribution as a function of $1 /\left(p^{2} \sin ^{3} \theta\right)$ of the tracks for data (solid points) compared to the nominal simulation (open points). A straight line fit to the data points is also shown. (b) The lifetime-signed impact parameter significance.

\begin{tabular}{|llll|}
\hline & Impact parameter & Standard vertex & Loose vertex \\
\hline$p_{\mathrm{T}}$ & $>1 \mathrm{GeV}$ & $>0.5 \mathrm{GeV}$ & $>0.5 \mathrm{GeV}$ \\
$d_{0}$ & $<1 \mathrm{~mm}$ & $<2 \mathrm{~mm}$ & $<10 \mathrm{~mm}$ \\
$z_{0} \sin \theta$ & $<1.5 \mathrm{~mm}$ & $<2 \mathrm{~mm}$ & $<50 \mathrm{~mm}$ \\
\hline
\end{tabular}

Table 2. Track selection criteria used for the impact parameter and secondary vertex tagging algorithms.

The transverse impact parameter, $d_{0}$, is a key variable for discriminating tracks originating from displaced vertices from those originating from the primary vertex. For studies of track impact parameters the $d_{0}$ was calculated with respect to a primary vertex which was fitted excluding that track in order to remove bias.

In order to study the effect of material on the $d_{0}$ resolution, figure 12(a) shows $\sigma^{2}\left(d_{0}\right)$ versus $1 /\left(p^{2} \sin ^{3} \theta\right)$ for data and simulation using all selected charged particle tracks. The quantity $\sigma\left(d_{0}\right)$ is determined by fitting the $d_{0}$ distribution in each bin of $1 /\left(p^{2} \sin ^{3} \theta\right)$ with a Gaussian within $\pm 2 \sigma\left(d_{0}\right)$ about its mean. The data lie approximately on a straight line, as is expected if the scattering material is on a cylinder and the match of the slope with the simulation implies a good description of the material of the inner detector. It should be noted that the intercept on the $y$ axis has a contribution from the primary vertex resolution.

The track selection for the $b$-tagging algorithms is designed to select well-measured particles and reject badly measured tracks, tracks from long-lived particles $\left(K_{\mathrm{S}}^{0}, \Lambda\right.$ and other hyperon decays), and particles arising from material interactions such as photon conversions or hadronic interactions.

The track selection used by the impact parameter tagging algorithm is summarized in the first column of table 2. Slightly different selections are used by the secondary vertex algorithm (second column of table 2).

Calorimeter jets (see section 7) are the reconstructed objects the tagging algorithms 


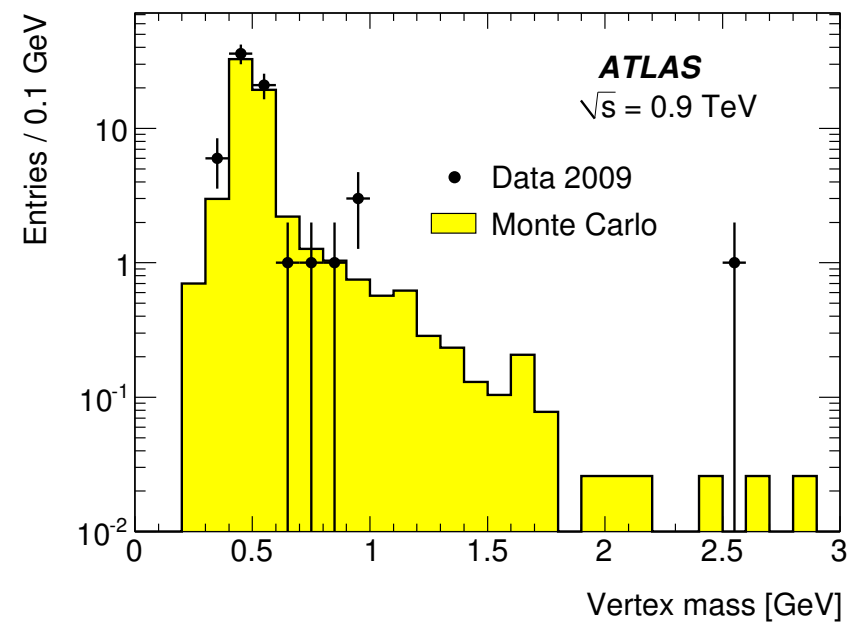

Figure 13. The vertex mass distribution for all secondary vertices with positive decay length selected in data. The expectation from simulated events, normalized to the number of jets in the data, is superimposed.

are typically applied to. Their direction is taken as estimator of the putative heavy flavour hadron direction. The impact parameter is then signed by whether the track perigee, relative to the jet direction, suggests a positive or negative flight distance. The distribution of the lifetime-signed impact parameter significance for tracks in jets is shown in figure 12(b).

Reconstructing explicitly the secondary decay vertices of heavy flavour hadrons adds substantial tagging information. There are expected to be few b-quarks which can be tagged in this data set, so the algorithm was run with the standard as well as loose settings, as described in the second and third columns of table 2, respectively. The loose setting selects vertices originating from $K_{\mathrm{S}}^{0}$ as well as from b-hadron decays whereas in the standard configuration any pair of tracks consistent with a $K_{\mathrm{S}}^{0}, \Lambda$ or photon conversion is explicitly removed.

Secondary vertices are reconstructed in an inclusive way starting from two-track vertices which are merged into a common vertex. Tracks giving large $\chi^{2}$ contributions are then iteratively removed until the reconstructed vertex fulfils certain quality criteria.

The mass distribution of the resulting vertices for the loose configuration, assuming a pion mass for each track, is shown in figure 13.

Running the algorithm in the standard configuration results in the reconstruction of 9 secondary vertices with positive decay length significance. This is in good agreement with the $8.9 \pm 0.5$ (stat.) vertices expected from the same number of jets, 10503 , in nondiffractive minimum-bias simulation. The vertices reconstructed with the standard version of the tagging algorithm are predominantly those with higher masses as the low-mass region is dominated by $K_{\mathrm{S}}^{0}$ mesons.

An event display of the highest-mass candidate is shown in figure 14. The secondary vertex consists of five tracks and has a mass of $2.5 \mathrm{GeV}$. The vertex is significantly displaced from the primary vertex, with a signed decay length significance $L / \sigma(L)=22$. From the 


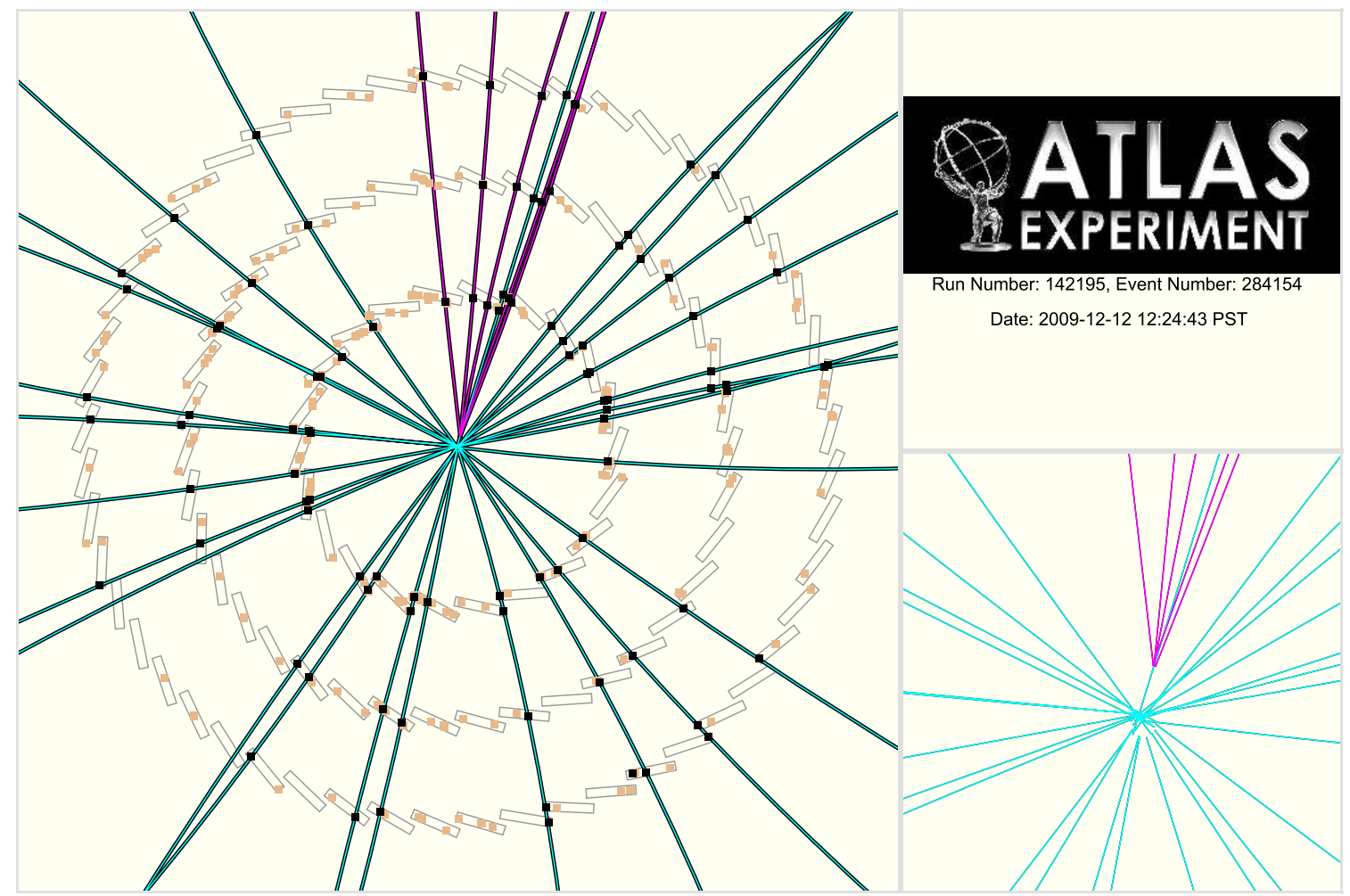

Figure 14. An event containing a secondary vertex selected by the secondary vertex algorithm. The pixel detector can be seen on the left and an expansion of the vertex region on the right. Unassociated hits, in a lighter colour, are predominantly due to unreconstructed particles such as those with transverse momenta below $0.5 \mathrm{GeV}$.

vertex mass, momentum and $L$ a proper lifetime of $3.1 \mathrm{ps}$ is estimated. The data was also tested by the impact-parameter based b-tagging algorithm and this jet is assigned a probability below $10^{-4}$ for originating from a light quark jet.

\subsection{Particle identification using transition radiation}

The TRT provides substantial discrimination between electrons and pions over the wide energy range between 1 and $200 \mathrm{GeV}$ by utilizing transition radiation in foils and fibres. The readout discriminates at two thresholds, the lower set to register minimum-ionising particles and the higher intended for transition radiation (TR) photon interactions. The fraction of high-threshold TR hits as a function of the relativistic $\gamma$ factor is shown in figure 15 for particles in the forward region. This region is displayed because there are more conversion candidates and they have higher momenta than in the barrel.

The high- $\gamma$ part of the distribution is constructed using electrons from photon conversions while the low- $\gamma$ component is made using charged particle tracks with a hit in the B-layer and treating them as pions. All tracks are required to have at least 20 hits in the TRT. The photon conversions are found similarly to those in section 6.7 with at least one silicon hit, but the transition radiation electron identification was not applied to the electron that was being plotted. To ensure high purity (about 98\%), the conversion 


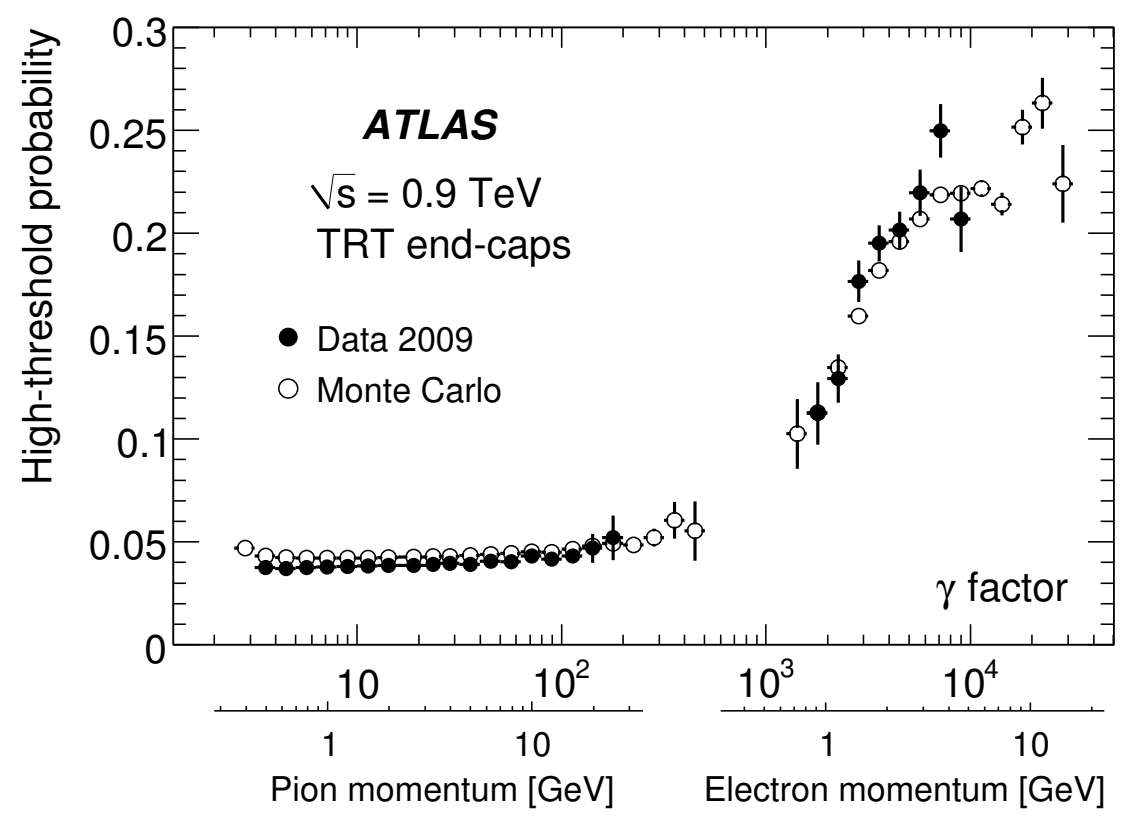

Figure 15. The fraction of high-threshold transition radiation hits on tracks as a function of the relativistic $\gamma$ factor (see text for details).

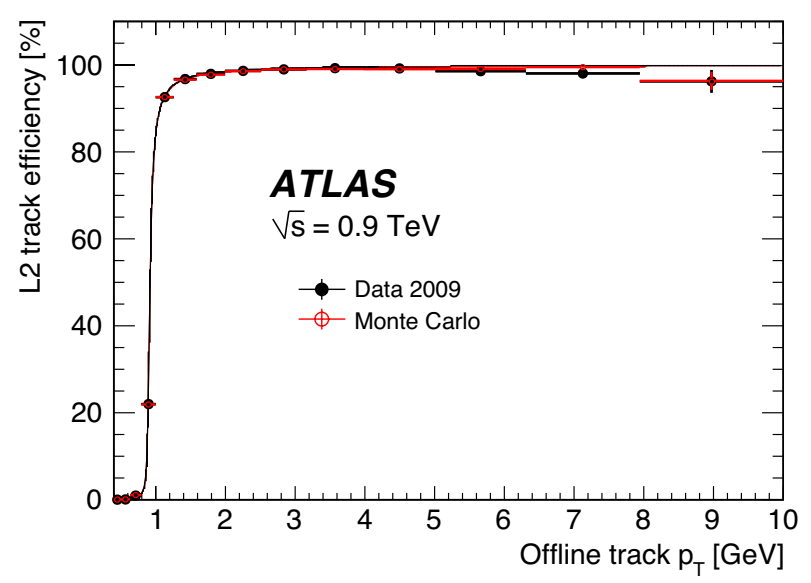

Figure 16. The efficiency for reconstruction of a L2 track candidate as a function of the $p_{\mathrm{T}}$ of the matched offline track in data and Monte Carlo simulation. A fit of the threshold curve is superimposed.

candidates are also required to have a vertex more than $40 \mathrm{~mm}$ away from the beam axis. The pion sample excludes any photon conversion candidate tracks.

\subsection{Tracking efficiency for Level-2 trigger}

The L2 track trigger is one component of the HLT whose performance can be tested with current data. The trigger runs custom track reconstruction algorithms at L2, designed to produce fast and efficient tracking using all tracking subdetectors. Tracking information forms an integral part of many ATLAS triggers including electron, muon and tau signa- 
tures [16]. These use L1 information to specify a region of interest to examine. In the 2009 data there were few high- $p_{\mathrm{T}}$ objects, so the results here are taken from a mode which searches for tracks across the entire tracking detector and is intended for B-physics and beam-position determination at L2.

Offline tracks with $\left|d_{0}\right|<1.5 \mathrm{~mm}$ and $\left|z_{0}\right|<200 \mathrm{~mm}$ are matched to L2 tracks if they are within $\Delta R=\sqrt{\Delta \eta^{2}+\Delta \phi^{2}}<0.1$. The efficiency is defined as the fraction of offline tracks which are matched and is shown in figure 16 as a function of the track $p_{\mathrm{T}}$.

\section{$6 \quad$ Electrons and photons}

The electron and photon reconstruction and identification algorithms used in ATLAS are designed to achieve a large background rejection and a high and uniform efficiency over the full acceptance of the detector for transverse energies above $20 \mathrm{GeV}$. Using these algorithms on the $0.9 \mathrm{TeV}$ data, a significant number of low- $p_{\mathrm{T}}$ electron and photon candidates were reconstructed. The measurements provide a quantitative test of both the algorithms themselves and the reliability of the performance predictions in the transverse energy range from the reconstruction threshold of $2.5 \mathrm{GeV}$ to about $10 \mathrm{GeV}$.

The electromagnetic calorimeter (EM) consists of the barrel (EMB) and two end-caps (EMEC). The barrel covers the pseudorapidity range $|\eta|<1.475$; the end-cap calorimeters cover $1.375<|\eta|<3.2$. In the forward direction energy measurements for both electromagnetic and hadronic showers are provided by the Forward Calorimeter (FCal) in the range $3.1<|\eta|<4.9$. The hadronic calorimetry in the range $|\eta|<1.7$ is provided by the scintillator-tile calorimeter (Tile). For $1.5<|\eta|<3.2$ hadronic showers are measured by the hadronic end-caps (HEC), which use LAr with a copper absorber.

The e $/ \gamma$ algorithms make use of the fine segmentation of the EM calorimeter in both the lateral and longitudinal directions of the showers [1]. At high energy, most of the EM shower energy is collected in the second layer which has a lateral granularity of $0.025 \times 0.025$ in $\eta \times \phi$ space. The first layer consists of finer-grained strips in the $\eta$-direction (with a coarser granularity in $\phi$ ), which improves $\gamma-\pi^{0}$ discrimination. A third layer measures the tails of very highly energetic EM showers and helps in rejecting hadron showers. In the range $|\eta|<1.8$ these three layers are complemented by a presampler layer placed in front with coarse granularity to correct for energy lost in the material before the calorimeter.

The algorithms also make use of the precise track reconstruction provided by the inner detector. The TRT also provides substantial discriminating power between electrons and pions over a wide energy range (between 1 and $200 \mathrm{GeV}$ ). The Pixel B-layer provides precision vertexing and significant rejection of photon conversions through the requirement of a track with a hit in this layer.

\subsection{Electron and photon reconstruction}

The basic algorithms for electron and photon reconstruction are described in detail in ref. [16]. The first stage of the search for EM objects is to look for significant deposits in the EM calorimeter cells inside a sliding window as it is moved across the detector. The size of the sliding window cluster depends on the type of candidate (electron, unconverted or 
converted photon) and the location (barrel, end-caps). The cluster energy is calculated from the amplitudes observed in the cells of the three longitudinal layers of the EM calorimeter and of the presampler (where present). The calculation sums the weighted energies in these compartments, then takes into account several corrections for shower depth, lateral and longitudinal leakage, local modulation etc. The weights and correction coefficients were parameterized from beam-tests [1] and simulation.

Electrons are reconstructed from the clusters if there is a suitable match with a particle track of $p_{\mathrm{T}}>0.5 \mathrm{GeV}$. The best track is the one with an extrapolation closest in $(\eta, \phi)$ to the cluster barycentre (the energy-weighted mean position) in the middle EM calorimeter layer. Similarly, photons are reconstructed from the clusters if there is no reconstructed track matched to the cluster (unconverted photon candidates) or if there is a reconstructed conversion vertex matched to the cluster (converted photon candidates). "Single track conversions" (identified via tracks lacking a hit in the B-layer) are also taken into account. First, electron candidates with a cluster $|\eta|<2.47$ and photons with cluster $|\eta|<2.37$ are selected and investigated (the cluster $\eta$ is defined here as the barycentre of the cluster cells in the middle layer of the EM calorimeter). Electron and photon candidates in the EM calorimeter transition region $1.37<|\eta|<1.52$ are not considered. At this stage, 879 electron and 1694 photon candidates are reconstructed in the data with $E_{T}$ above $2.5 \mathrm{GeV}$.

\subsection{Electron and photon identification}

The isolated electron and photon identification algorithms rely on selections based on variables which provide good separation between electrons/photons and fake signatures from hadronic jets. These variables include information from the calorimeter and, in the case of electrons, tracker and combined calorimeter/tracker information. There are three classes of electrons defined: loose, medium and tight, and two for photons: loose and tight. The selection criteria were optimized in bins of $E_{T}$ and $\eta$, separately for electrons, unconverted and converted photons.

The loose selection criteria are based on the shower shape and are common to electrons and photons. For electrons, the medium requirements make use of the track information while in the tight ones the particle track selections are more stringent and use the particle identification capability of the TRT. For photons the tight selection criteria make full use of the EM calorimeter strip layer information, mainly to reject merged photon pairs from high energy $\pi^{0}$ 's.

In the following all reconstructed electron and photon candidates with cluster $E_{T}>$ $2.5 \mathrm{GeV}$ at the sliding window level are considered.

\subsection{Electron candidates}

Figure 17 displays, for all of the 879 electron candidates from 384186 events, the transverse energy and pseudorapidity spectra. Table 3 presents the percentage of these candidates which pass the successive selection criteria both for data and simulation. These criteria were not optimized for such low-energy electron candidates (see section 6.2). Both figure 17 and table 3 show similar behaviour in data and simulation. The remaining discrepancies in the first stages of background rejection may be related to the small differences observed 


\begin{tabular}{|lcccc|}
\hline & \multicolumn{2}{c|}{ Electron candidates } & \multicolumn{2}{c|}{ Photon candidates } \\
\cline { 2 - 5 } & Data (\%) & MC (\%) & Data (\%) & MC (\%) \\
\hline Loose & $46.5 \pm 1.7$ & $50.9 \pm 0.2$ & $25.4 \pm 1.0$ & $30.5 \pm 0.1$ \\
Medium & $10.6 \pm 1.0$ & $13.1 \pm 0.2$ & n.a. & n.a. \\
Tight & $2.3 \pm 0.5$ & $2.4 \pm 0.1$ & $4.1 \pm 0.5$ & $6.6 \pm 0.1$ \\
\hline
\end{tabular}

Table 3. The fraction of electron and photon candidates passing the different selection criteria, compared to those predicted by Monte Carlo (MC). Statistical error are quoted.
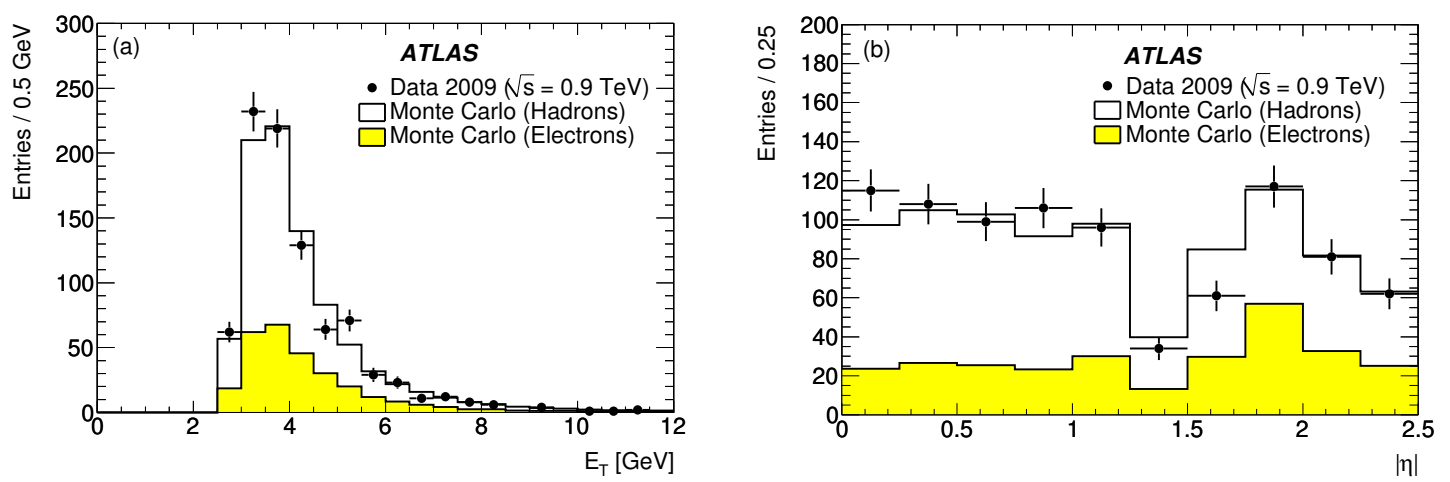

Figure 17. Distribution of cluster $E_{T}$ (a) and $|\eta|$ (b) for all selected electron candidates. The simulation is normalized to the number of data events.

in shower variables (see section 6.6.1 below). In figure 17(b) the drop in efficiency around $|\eta|=1.5$ corresponds to the barrel/end-cap transition.

In these figures the Monte Carlo prediction is sub-divided into its two main components: hadrons and real electrons. The latter is largely dominated by electrons from photon conversions, but also includes a small fraction $(\sim 3 \%)$ of electrons from other sources, such as Dalitz decays, and an even smaller one (below 1\%) of electrons from b, $\mathrm{c} \rightarrow$ e decays. There are twenty electron candidates passing the tight selections in the data. Approximately $15 \%$ of such candidates in the Monte Carlo are from heavy flavour decays.

\subsection{Photon candidates}

Transverse energy and pseudorapidity spectra for all 1694 photon candidates are displayed in figure 18. Table 3 presents the percentage of photon candidates, as a function of the selection level applied. Of the selected candidates, $14 \%$ are reconstructed as converted photons and almost all of these, $\sim 98 \%$, are also selected as electron candidates.

The Monte Carlo prediction is sub-divided in this case into four components of decreasing importance: approximately $71 \%$ of the candidates correspond to photons from $\pi^{0}$ decay, whereas $\sim 14 \%$ are from $\eta, \eta^{\prime}$ or $\omega$ decays into photons; $\sim 14 \%$ are from other hadrons with complex decay processes and particles interacting in the tracker material. At these energies, only a very small fraction, $\sim 1 \%$, of all photon candidates are expected to be primary products of the hard scattering. 

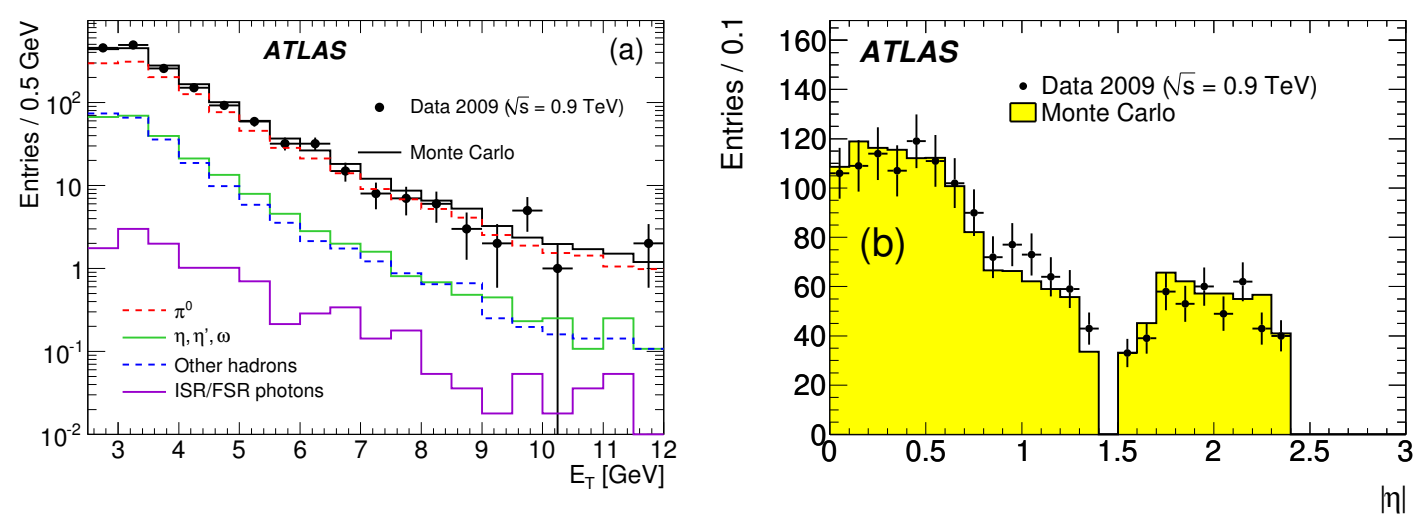

Figure 18. Cluster $E_{T}$ (a) and $|\eta|$ (b) for all selected photon candidates. The simulation is normalized to the number of data events.

\subsection{First-level electron and photon trigger performance}

The L1 $e / \gamma$ selection algorithm searches for narrow, high- $E_{T}$ electromagnetic showers and does not separate electrons from photons. The primitives for this algorithm are towers which sum the transverse energies of all electromagnetic calorimeter cells in $\Delta \eta \times \Delta \phi=$ $0.1 \times 0.1$. The trigger examines adjacent pairs of towers and tests their total energy against several trigger thresholds. Isolation requirements were not yet employed. The lowest threshold $e / \gamma$ trigger for the 2009 data-taking period required a transverse energy of at least $4 \mathrm{GeV}$.

Clusters consistent with originating from an electron or photon are selected by requiring at least $30 \%$ of the cluster energy to be deposited in the second layer of the electromagnetic calorimeter, where the maximum of an electromagnetic shower is expected. The selected $e / \gamma$ candidates are matched to L1 clusters in $\eta$ and $\phi$ by requiring $\Delta R<0.15$. The efficiency is then calculated from the fraction of reconstructed clusters which have a matching L1 cluster.

The resulting L1 trigger efficiency for the lowest threshold component is shown in figure 19. The sharpness of the efficiency turn-on curve around threshold agrees with the Monte Carlo expectation. Low energy reconstructed clusters occasionally fire the trigger, especially when the coarser granularity used at L1 merges two separate offline clusters.

\subsection{Electron and photon identification variables}

\subsubsection{Calorimeter variables}

In this section, various calorimeter-based quantities are displayed for the photon candidates. These are preferred to the similar electron distributions because of the higher purity.

Figure 20 illustrates the longitudinal development of the shower in the successive layers of the EM calorimeter, based on the measured layer energies before corrections are applied. For the observed photon candidates, which in simulation are predominantly from $\pi^{0}$ decays, the energy is deposited in earlier calorimeter layers than typical for high energy photons. In the presampler part, the simulation points are higher than the data for fractions above 


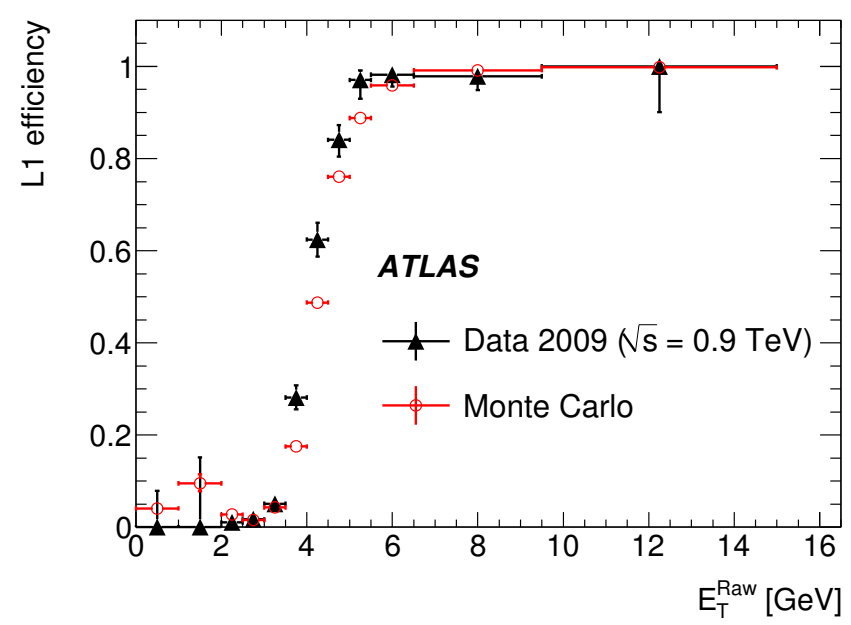

Figure 19. Efficiency for the lowest threshold L1 electromagnetic trigger, a nominal $4 \mathrm{GeV}$, as a function of the uncalibrated offline cluster transverse energy. The turn-on is shown for data (solid triangles) and non-diffractive minimum-bias simulation (open circles).

0.6. This is at least in part because the presampler simulation does not describe the recombination of electron-positron pairs by highly ionizing hadrons or nuclear fragments that lower the LAr response, an effect which is included in the accordion calorimeter simulation. This feature also explains the observed disagreement in the first bins for the fractions in the other layers, since the various fractions are correlated.

Several variables are used to quantify the lateral development of the shower. From these, the distribution of $w_{2}$, the shower width measured in the second layer of the EM calorimeter, is shown in figure 21(a). The shower width $w_{2}$ is slightly larger in the data. Preliminary studies show that including the cross-talk between neighbouring middle layer cells $(\sim 0.5 \%)[17]$ in the simulation explains part of the observed difference.

The distribution of two variables used for $\pi^{0} / \gamma$ separation in the tight photon selection, $E_{\text {ratio }}$ and $w_{s 3}$, are shown in figures $21(\mathrm{~b})$ and $21(\mathrm{c}) . E_{\text {ratio }}$ is the difference of the highest and second highest strip energies, divided by their sum. $w_{s 3}$ is the shower width measured in three strips around the maximum energy strip. For this variable the data show a slightly wider profile than the simulation, although in this case the simulation already includes the measured cross-talk. In general, all the shower shape variables show good agreement between data and simulation.

\subsubsection{Tracking and track-cluster matching variables}

Electron and converted photon identification rely heavily on tracking performance. Figure 22 illustrates two of the track-calorimeter matching variables used in the identification of electron candidates in data and simulation. For simulation, hadrons and real electrons are shown separately. Figure 22(a) shows the difference in $\eta, \Delta \eta_{1}$, between the track extrapolated to the strip layer of the EM calorimeter and the barycentre of the cell energies in this layer. Figure 22(b) shows the difference in azimuth, $\Delta \phi_{2}$, between the track extrap- 

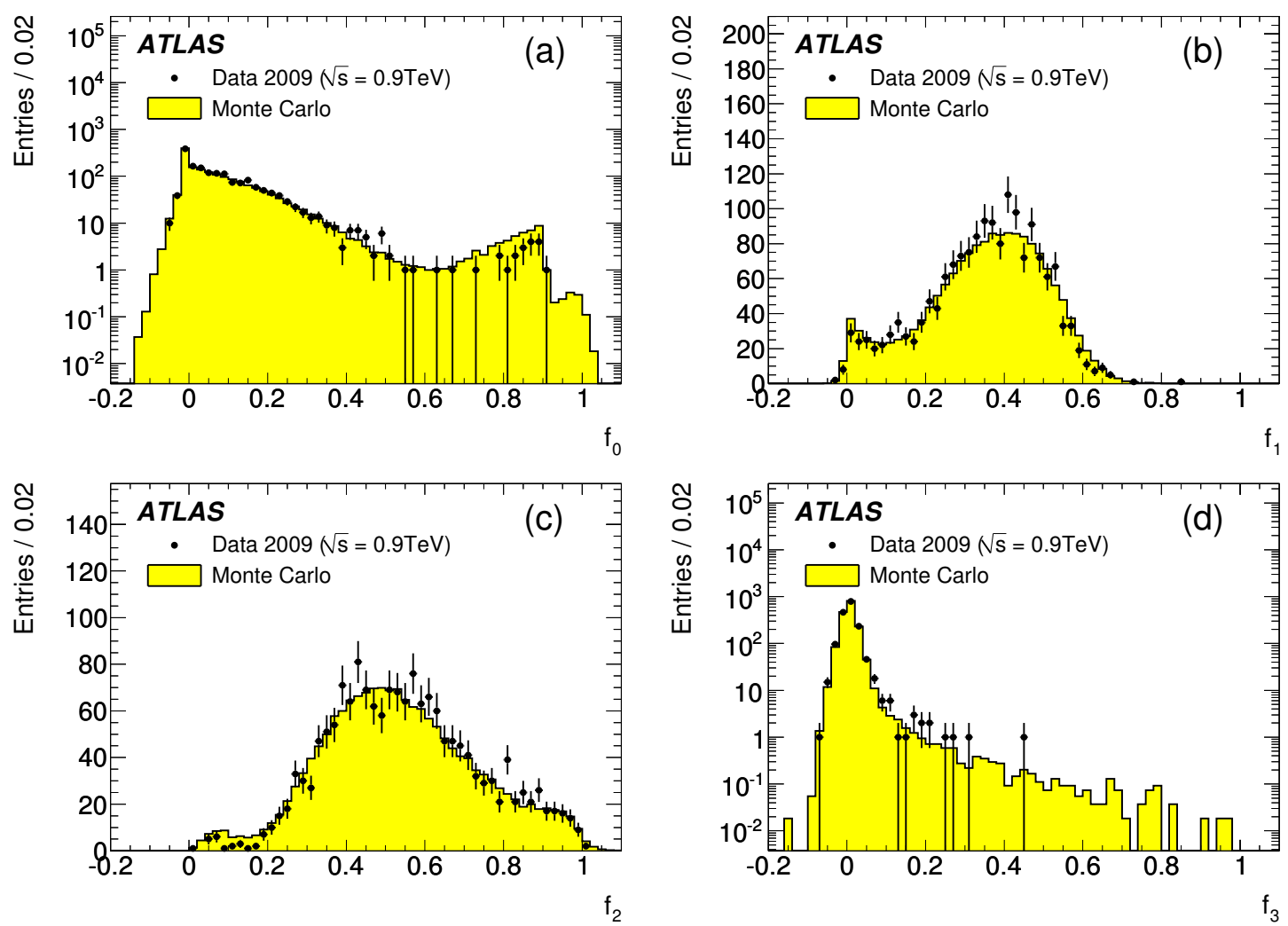

Figure 20. Fraction of energy deposited by photon candidates with $E_{T}>2.5 \mathrm{GeV}$ in each layer of the electromagnetic calorimeter for data and simulation. These fractions are labelled as (a) $f_{0}$ for the presampler layer, (b) $f_{1}$ for the strip layer, (c) $f_{2}$ for the middle layer and $(d) f_{3}$ for the back layer. Fractions can be negative due to noise fluctuations. The simulation is normalized to the number of data events.

olated to the middle layer of the EM calorimeter and the barycentre of the cell energies in this layer. This variable is signed by the charge of the particle to account for the position of any radiated photons with respect to the track curvature, and an asymmetric cut is applied in the selection. The asymmetric tails at large negative values of $\Delta \phi_{2}$ are more pronounced for the electrons than for the hadrons.

Figure 23 shows a comparison of four of the tracking variables between data and simulation for all electron candidates. Figures 23(a) and 23(b) show the numbers of hits on the tracks in the Pixel and SCT detectors, respectively. The fraction of high-threshold TRT hits belonging to the track for electron candidates with $|\eta|<2.0$ and with a total number of TRT hits larger than ten is shown in figure 23(c). At these low energies the transition radiation yield of electrons is not optimal and yet a very clear difference can be seen between the distributions expected for hadrons and for electrons from conversions. Finally, figure 23(d) shows the distribution of the transverse impact parameter, $d_{0}$, of the electron track with respect to the reconstructed primary vertex position in the transverse plane; whereas the hadrons in the simulation display a distribution peaked around zero with a resolution of $\sim 100 \mu \mathrm{m}$, the electrons from conversions have large impact parameters. 

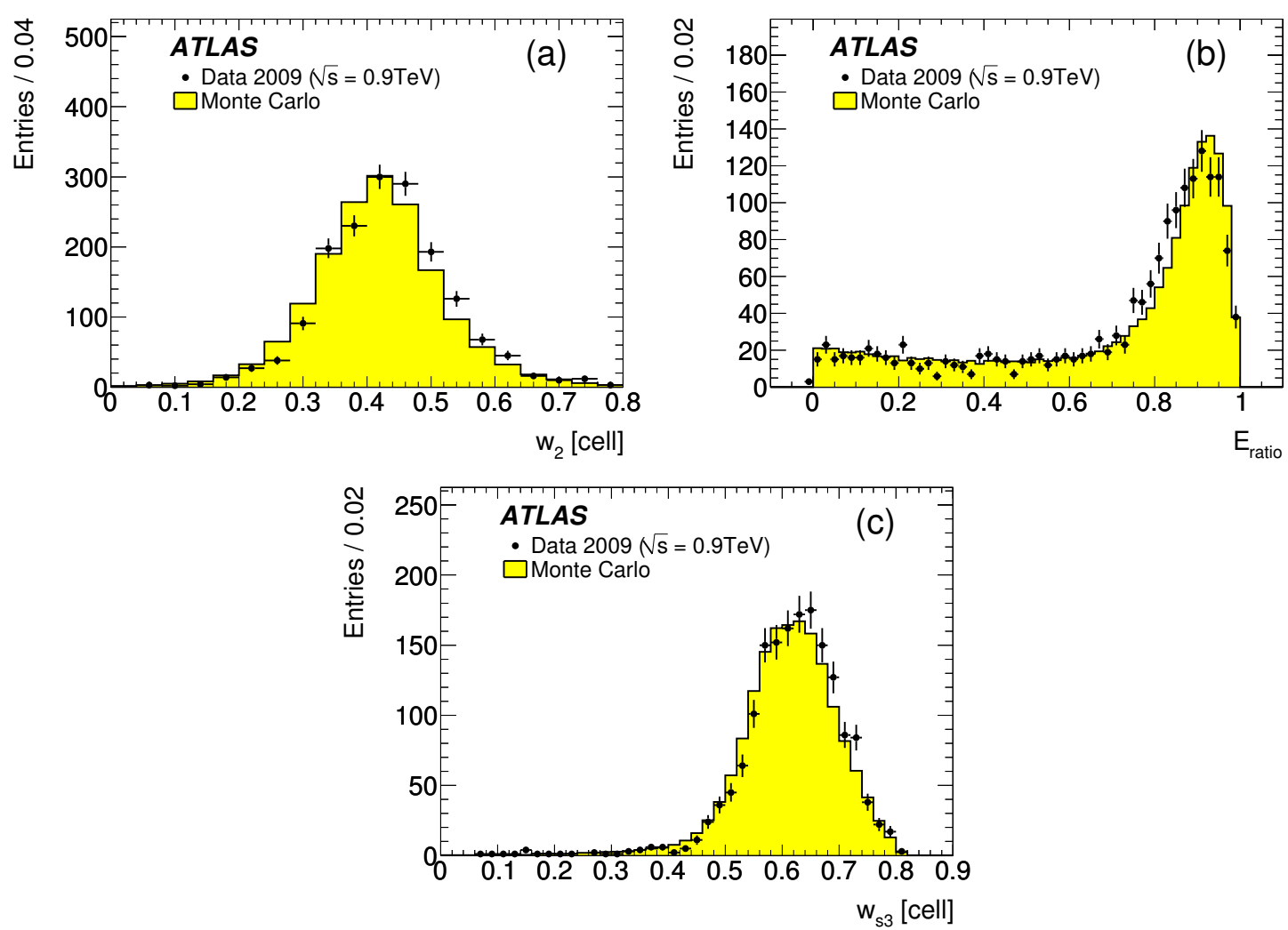

Figure 21. Distributions of calorimeter variables compared between data and simulation for all photon candidates with $p_{\mathrm{T}}$ above $2.5 \mathrm{GeV}$. Shown are the shower width in the middle layer of the EM calorimeter, $w_{2}(\mathrm{a})$, and the variables $E_{\text {ratio }}(\mathrm{b})$ and $w_{s 3}(\mathrm{c})$, which characterize the shower shape in the first (strips) EM layer. The simulation is normalized to the number of data events.
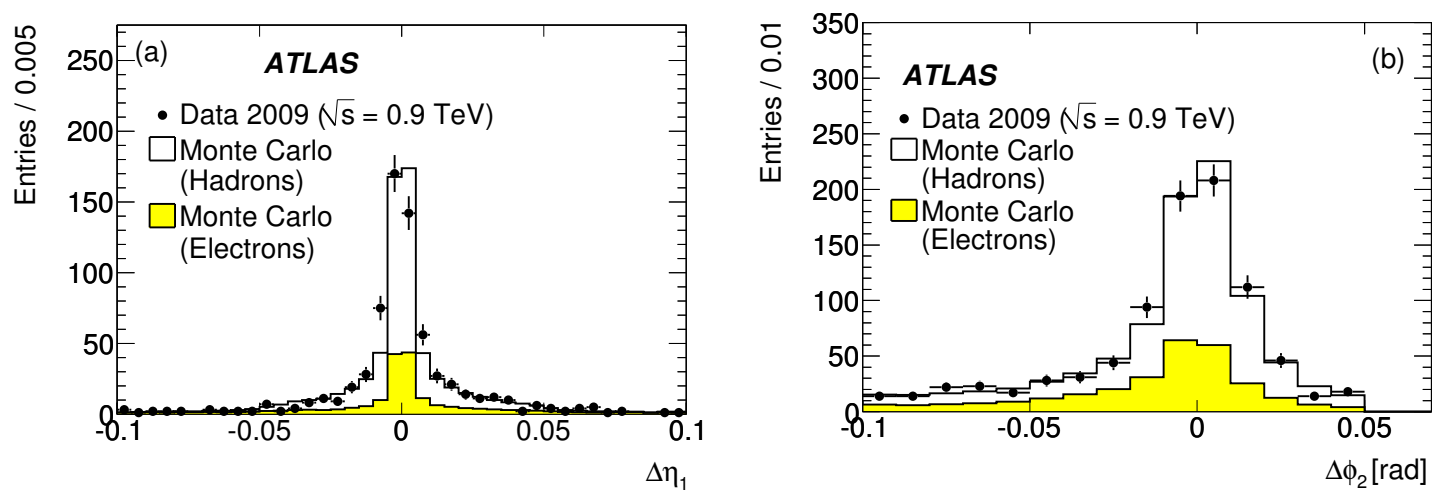

Figure 22. Distributions of track-calorimeter matching variables for all electron candidates compared between data and simulation. (a) shows the difference in $\eta$ in the first calorimeter layer (see section 6.6.2) and (b) shows the match in charge-signed- $\phi$ in the second. The simulation is normalized to the number of data events.

The agreement between data and simulation is good, despite the complications expected at these low energies due to material effects and track reconstruction inefficiencies. 

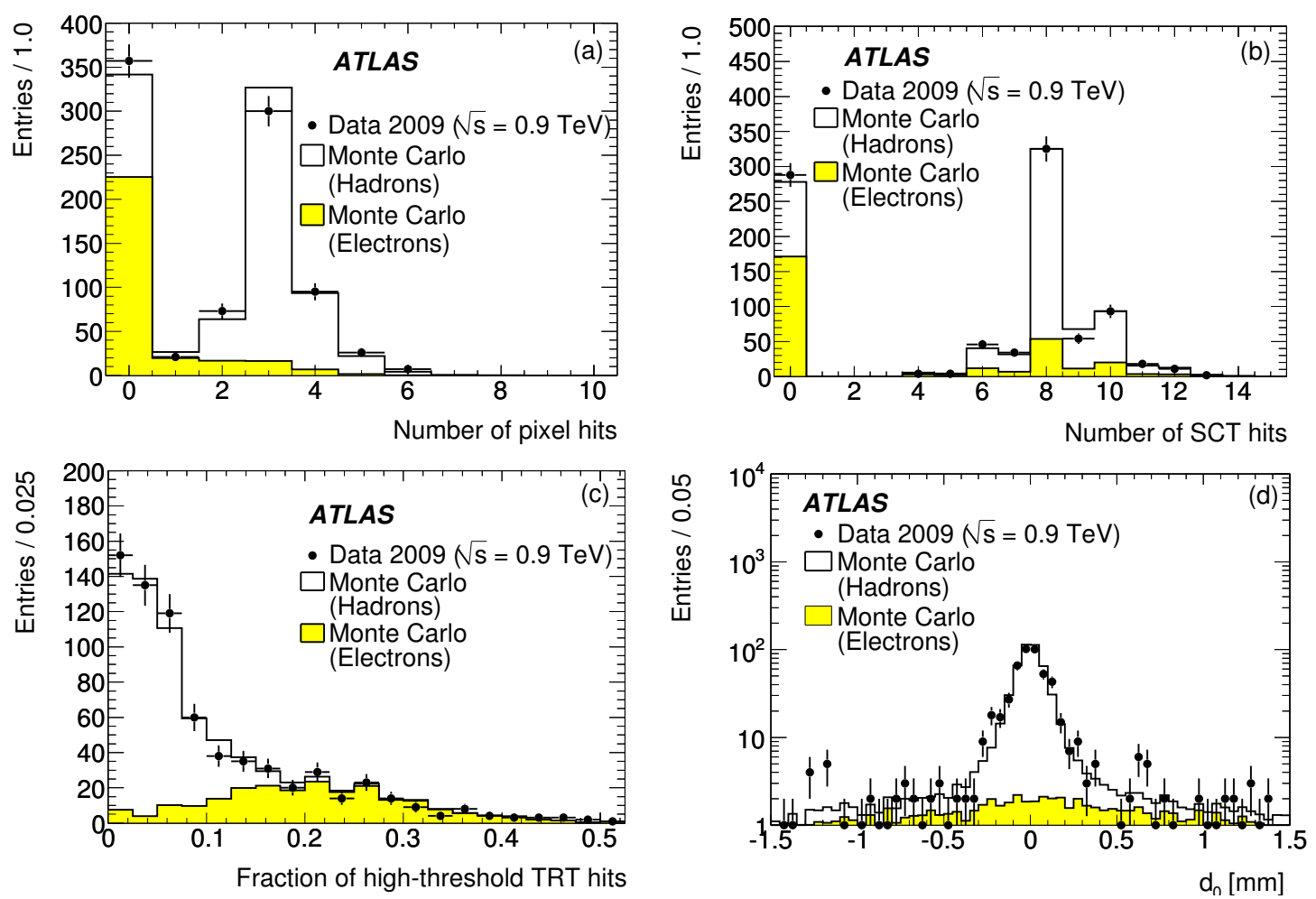

Figure 23. Distributions of tracking variables for all electron candidates compared between data and simulation. The number of Pixel (a) and SCT (b) hits on the electron tracks are shown, the fraction of high-threshold TRT hits for candidates with $|\eta|<2.0$ and with a total number of TRT hits larger than ten (c), and the transverse impact parameter, $d_{0}$, with respect to the reconstructed primary vertex $(\mathrm{d})$. The simulation is normalized to the number of data events.
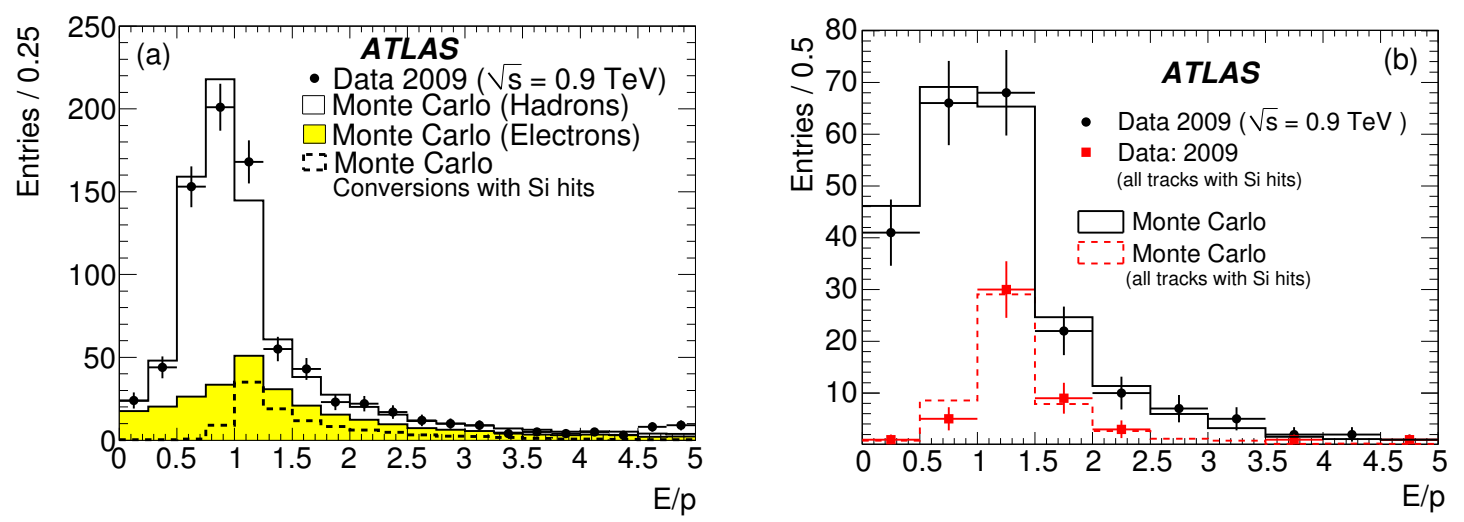

Figure 24. Ratio, $E / p$, between cluster energy and particle track momentum (a) for electron candidates and (b) for electrons from converted photons. In each case candidates with $p_{\mathrm{T}}$ above $2.5 \mathrm{GeV}$ in the calorimeter are shown. Sub-figure (a) is dominated by real electrons. The simulation is normalized to the number of data events.

Figure 24(a) shows the distribution of the ratio $E / p$ of cluster energy in the calorimeter to track momentum for all electron candidates and for data and simulation. Electrons from 
conversions have a broad $E / p$ distribution as their shortened tracks have a large momentum error. The hadron component peaks at values near unity: this behaviour, due to the selection bias for these hadrons, is also observed in the simulation. In a similar fashion, figure 24(b) shows the $E / p$ ratio of the reconstructed converted photon candidates, where the converted photon momentum is estimated from the combination of the particle momenta for double-track conversions and from the particle momentum measurement available for single-track conversions. Approximately $20 \%$ of the converted photon candidates are reconstructed as single-track conversions in this kinematic regime. Both the electron dominated and the hadron dominated distributions show good agrement with the simulation.

\subsubsection{Use of the TRT for electron identification}

As already discussed in section 6.3, the electron candidate data sample is expected to consist predominantly of two components: charged hadrons misreconstructed as electrons and electrons from photon conversions. These two components can be separated by using the measured fraction of high-threshold TRT hits on the electron tracks (see figure 23(c)). To perform such a measurement, the electron candidates are required to lie within the TRT acceptance, i.e. $|\eta|<2.0$, and to have a reconstructed track with a total of at least ten TRT hits.

The distribution of the fraction of high threshold hits has been fitted in 20 bins between 0 and 0.5 to extract the number of hadrons and electrons observed in the data. This relies upon the modelling of the response of the TRT to electrons and pions in the simulation. The sample of electron candidates considered here is predicted to contain $494 \pm 26$ electron candidates which are actually hadronic fakes and $226 \pm 21$ genuine electrons.

Two examples of comparisons between the shapes of variables extracted for each of the two components, using the method described above (on each bin individually), and the shapes predicted for each component are shown in figures 25 and 26, respectively, for two of the most sensitive variables: the fraction of the cluster energy measured in the strip layer and the ratio $E / p$. The $E / p$ distribution for electrons in both data and simulation shows a peak close to unity and a tail at large values from bremsstrahlung losses in the tracker material. The error estimates in these de-convolved plots come from toy Monte Carlo trials and their size reflects the power of the TRT detector for electron identification. The rates of electrons and hadrons and the relevant distributions agree with the Monte Carlo simulation for each species illustrating the quality of the simulation modelling.

\subsection{Photon conversions}

An accurate and high-granularity map of the inner detector material is necessary for a precise reconstruction of high-energy photons and electrons. The location of the conversion vertex can be used as a tool to map the position and amount of material of the inner detector. In the following, photon conversions are selected using only information from the inner detector, enabling the use of very low momentum particle track pairs. In addition, conversions give a source of electrons from which the TRT detector response can be determined.

The conversion reconstruction algorithm is described in detail elsewhere [16]. In the following, the basic steps of the algorithm are recalled together with an updated list of selection criteria. The algorithm begins by selecting single particle tracks with transverse 

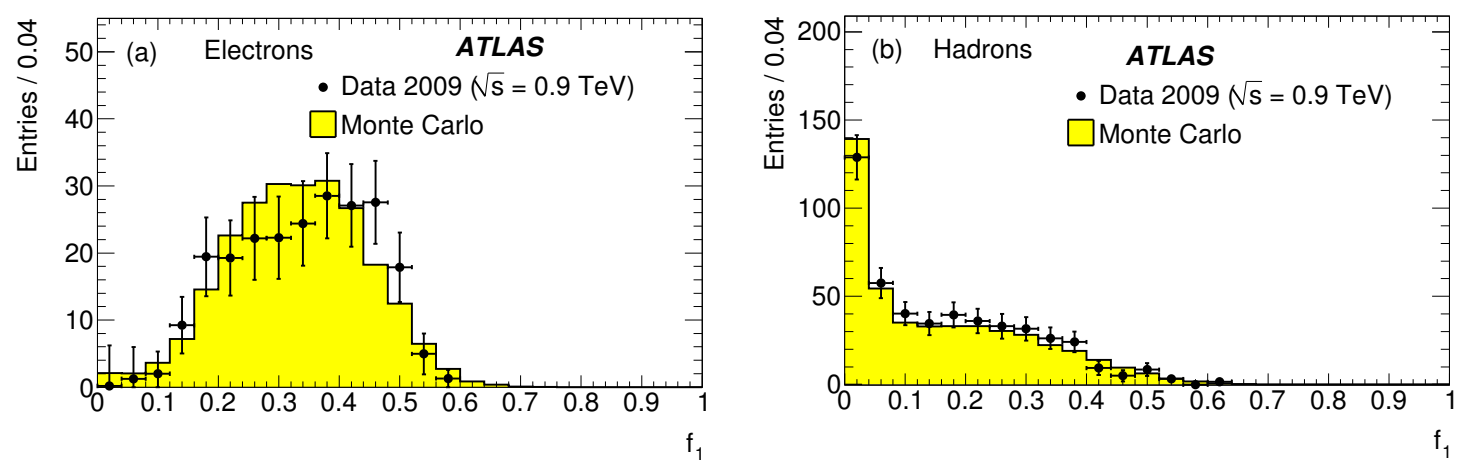

Figure 25. Distribution of the energy fraction in the strip layer of the EM calorimeter as extracted from data compared to the truth from simulation. The results are shown for both components of the electron candidates: electrons from conversions (a) and hadrons (b). The simulation is normalized to the number of data events.
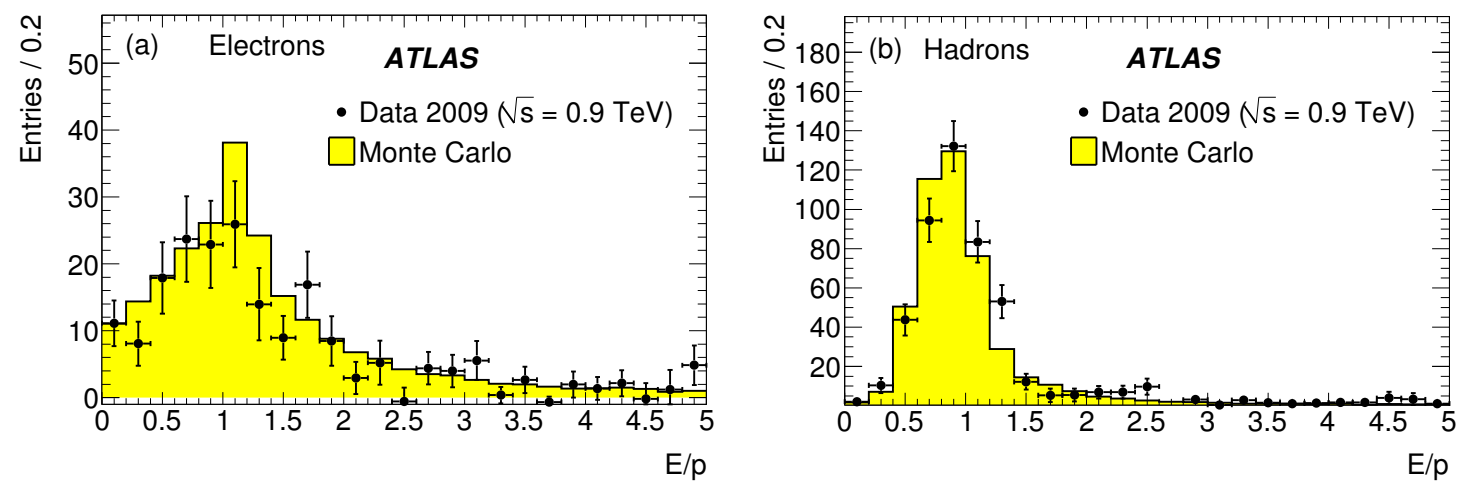

Figure 26. Distribution of the $E / p$ as extracted from data compared to the truth from simulation. The results are shown for both components of the electron candidates: electrons from conversions (a) and hadrons (b). The simulation is normalized to the number of data events.

momentum $p_{\mathrm{T}}>500 \mathrm{MeV}$. These tracks must have a probability of being an electron of more than $10 \%$, calculated using the particle identification capability of the TRT, see section 5.5.

Conversion candidates are then created by pairing oppositely charged particle tracks. The tracks are further required to be close in space and to have a small opening angle. The selected particle track pairs are then fitted to a common vertex with the constraint that they be parallel at the vertex. The final set of conversion candidates is selected based on the quality of the vertex fit which must have $\chi^{2}$ smaller than 50 .

The tracks used for the reconstruction of conversions may be stand-alone TRT tracks, or they may include silicon hits. In the data, 3662 vertices, $6.7 \%$ of the total, have two tracks with silicon information, to be compared with $10.4 \%$ in the simulation. This class of vertices have much less background than the total and the following results are drawn from them. Some properties of the candidates in data and Monte Carlo simulation are shown in figure 27. Given the complexity of the reconstruction of converted photons and the impact 

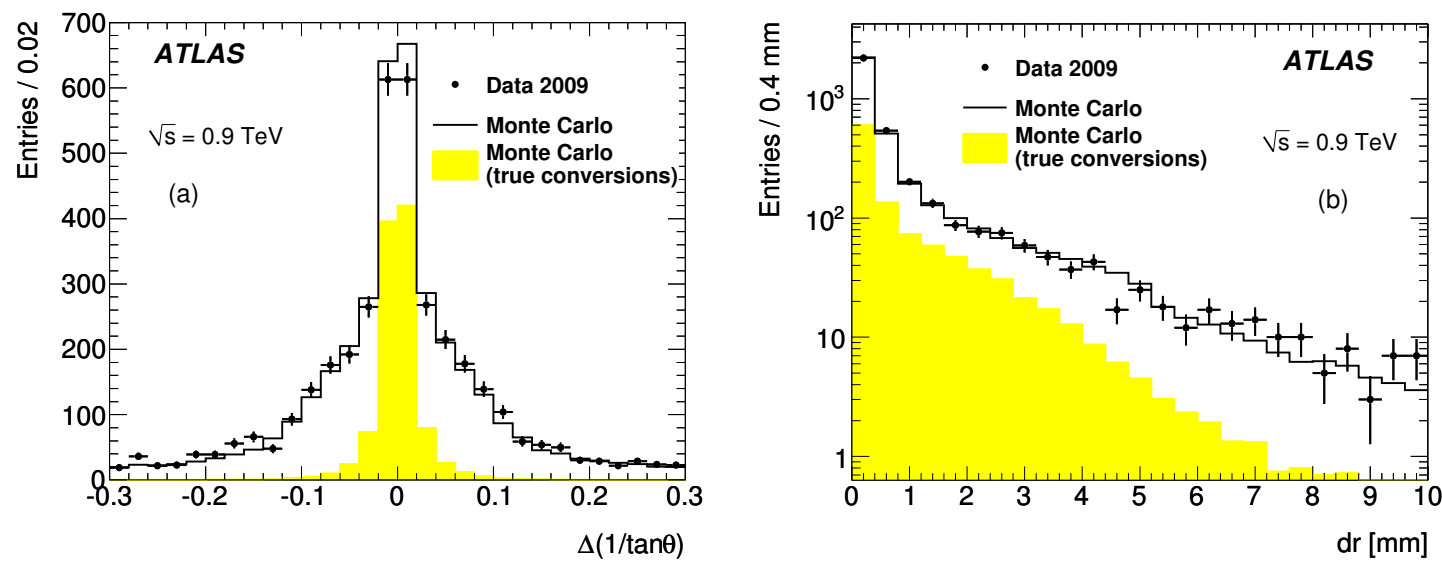

Figure 27. Comparison between converted photon candidates, for which both tracks have silicon hits, in data and non-diffractive minimum-bias Monte Carlo simulation. (a) Opening angle in the $r z$ plane between the two tracks $(\Delta(1 / \tan \theta))$; (b) 3D distance of closest approach between the two tracks, dr. The distributions are normalized to the same number of conversion candidates in data and Monte Carlo simulation.
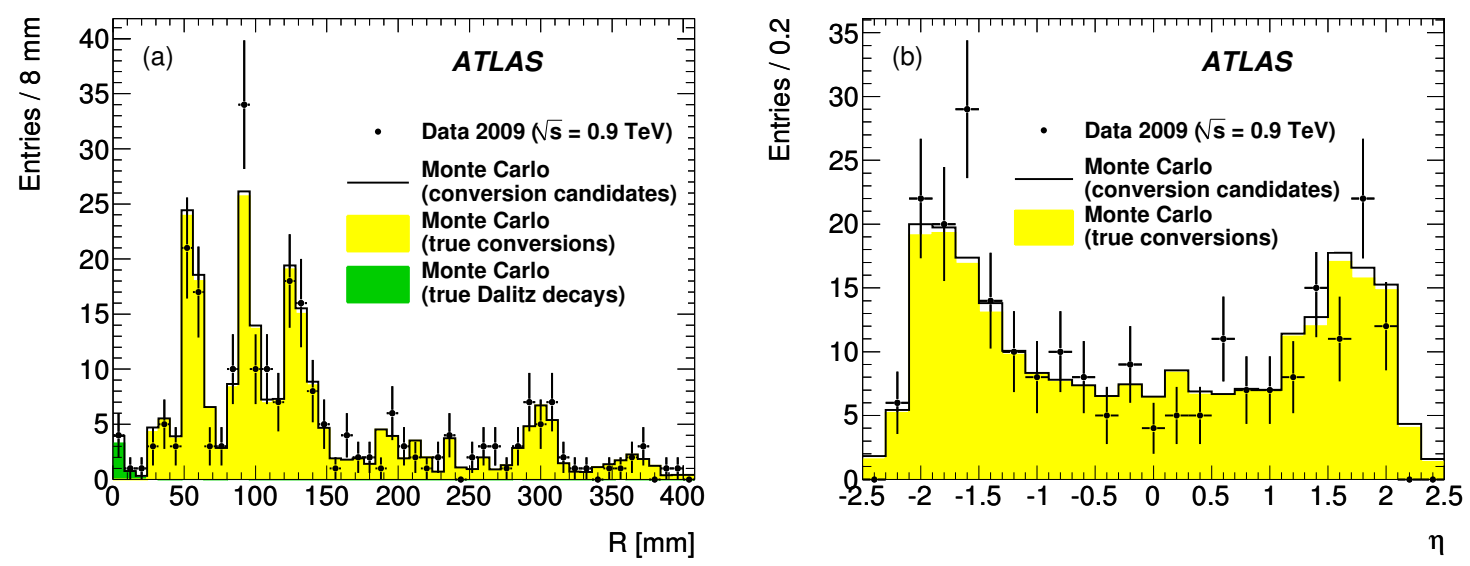

Figure 28. Distribution of conversion candidate radius, (a), and $\eta$, (b). The points show the distribution for data; the open histograms, the total from the Monte Carlo simulation and the filled component shows the expected contribution of true photon conversions. The contribution from the Dalitz decays of neutral mesons is shown in sub-figure (a). The Monte Carlo simulation is normalized to number of conversion candidates in the data, although in subsequent analysis normalization is to the number in the beam pipe.

of bremsstrahlung of the electrons in the tracker material, the consistency between the data and the simulation for the selection variables is good.

To measure the inner detector material the selection requirements are tightened to $>90 \%$ TR electron probability and vertex $\chi^{2}<5$.

Figure 28 shows the location in radius and $\eta$ of conversion vertices. The simulation was normalized to the same number of conversions as in the data and the agreement in shape is satisfactory. 


\begin{tabular}{|lccc|}
\hline & $N_{\text {reco }}$ & $\frac{X}{X_{0} \text { data }}$ & $\frac{X}{X_{0} \mathrm{MC}}$ \\
\hline Beam pipe & 9 & 0.00655 & 0.00655 \\
Pixel B-layer & 46 & $0.030 \pm 0.004$ & 0.032 \\
Pixel layer 1 & 65 & $0.035 \pm 0.004$ & 0.027 \\
Pixel layer 2 & 55 & $0.025 \pm 0.003$ & 0.023 \\
SCT layer 1 & 25 & $0.020 \pm 0.004$ & 0.016 \\
\hline
\end{tabular}

Table 4. $N_{\text {reco }}$ is the number of reconstructed conversions in each layer, and $X / X_{0 \text { data }}$ and $X / X_{0 \mathrm{MC}}$ represent the amount of material in the different volumes estimated from data and Monte Carlo, normalized by the number of reconstructed converted photons in the beam pipe, whose material is assumed to be correct. The normalization introduces an additional statistical uncertainty of $30 \%$ on $X / X_{0 \text { data }}$.

The amount of material, in multiples of the radiation length $X_{0}$, that the photons traverse can be calculated from the fraction of photon conversions seen in it given the reconstruction efficiency. The combinatorial background and the error in determining the conversion radius must be accounted for. To remove the dependence on the absolute flux of photons and the overall reconstruction efficiency, the rate is normalized to that seen in a well-understood reference material volume, which is chosen to be the beam pipe. As shown in figure 28 , there were only 9 conversions in this reference volume and so the absolute material determination has errors of at least 30\%. The agreement between data and Monte Carlo is presented in table 4.

\subsection{Reconstruction of $\pi^{0}$ and $\eta$ mesons}

For the analysis presented in this section, cells from the four layers are combined to form a cluster of size $\Delta \eta \times \Delta \phi=0.075 \times 0.125$, which corresponds to an area of $3 \times 5$ cells in the middle layer of the EM calorimeter. The EM cell clusters are reconstructed with a seed cell threshold $\left|E_{\text {cell }}\right|=4 \sigma$ (where $\sigma$ corresponds to the electronic noise in the cell) and with a cluster transverse energy $E_{T}>300 \mathrm{MeV}$ [16]. These clusters are used as photon candidates for $\pi^{0}$ and $\eta$ reconstruction.

The standard parameterization of energy response discussed in section 6.1 was performed for photons with $E_{T}>5 \mathrm{GeV}$. For the present study a dedicated parameterization was extracted from the minimum-bias simulation sample using low-energy photons coming only from $\pi^{0} \mathrm{~s}$.

\subsubsection{Extraction of $\pi^{0} \rightarrow \gamma \gamma$ signal}

In order to extract the $\pi^{0}$ signal from the combinatorial background, well measured photons were selected inside an acceptance of $|\eta|<2.37$, excluding a transition region $1.37<|\eta|<$ 1.52. The fraction of energy in the first layer, $E_{1} /\left(E_{1}+E_{2}+E_{3}\right)$, was required to be larger than 0.1 and the clusters were required to have a transverse energy, $E_{\mathrm{T}}$, above $400 \mathrm{MeV}$.

All pairs of photons with $p_{\mathrm{T}}^{\text {pair }}>900 \mathrm{MeV}$ are selected. There are about $8 \times 10^{5}$ of these in the data. 

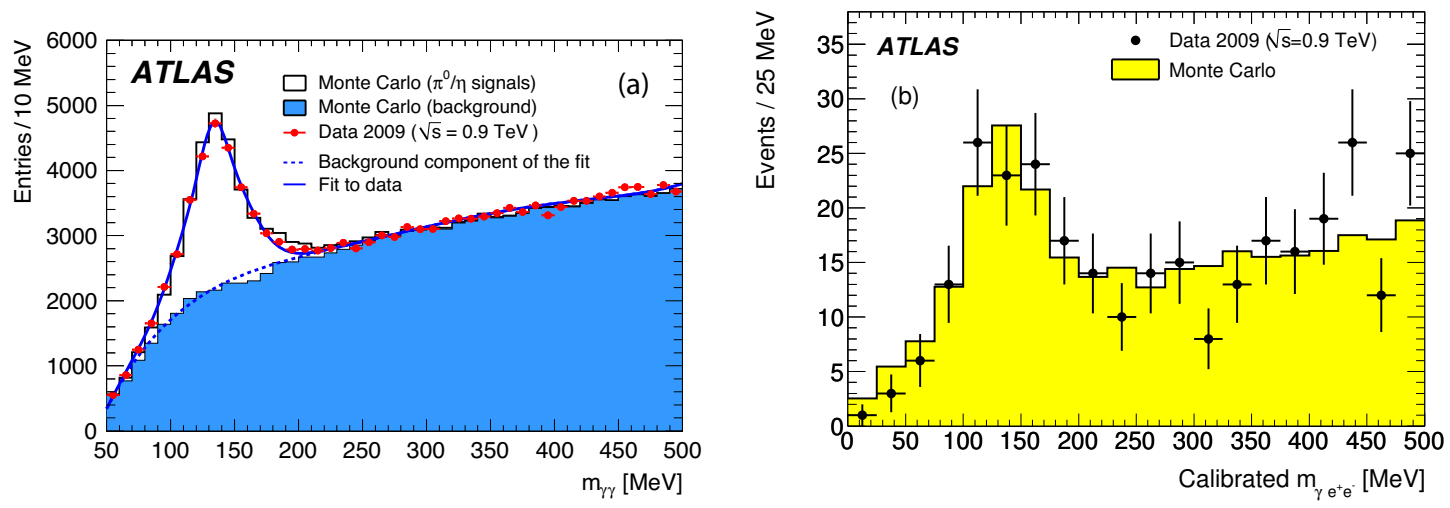

Figure 29. (a) Diphoton invariant mass distribution for the $\pi^{0}$ selection for data and Monte Carlo. The Monte Carlo is normalized to the same number of entries as the data. (b) Invariant mass distribution from one converted and one unconverted photon. The data are represented by points and the Monte Carlo simulations are shown as histograms.

\subsection{2 $\pi^{0}$ mass fit}

The invariant mass distribution of the photon pairs is shown in figure 29 for both data and Monte Carlo. The diphoton mass distribution is fitted using a maximum-likelihood fit. The signal is described by the sum of a Gaussian and a "Crystal-Ball function" [18], which are required to have the same mean. The combinatorial background is described with a $4^{\text {th }}$ order Chebyshev polynomial. The parameters of the signal and the background normalization are varied in the fit to the data, while the parameters of the polynomial were extracted from the Monte Carlo.

The fitted $\pi^{0}$ mass is $134.0 \pm 0.8 \mathrm{MeV}$ for the data and $132.9 \pm 0.2 \mathrm{MeV}$ for the Monte Carlo where the errors are statistical only. The mass resolution in the data is $24.0 \mathrm{MeV}$, to be compared with $25.2 \mathrm{MeV}$ in the simulation, and the number of $\pi^{0}$ 's is $(1.34 \pm 0.02)$ $\times 10^{4}$. This fit is sensitive to the modelling of the background shape near the $\pi^{0}$ mass. Varying the background shape under the peak leads to a differences of up to $1 \%$ for the fitted $\pi^{0}$ mass, up to $10 \%$ for the fitted $\pi^{0}$ mass resolution and up to $20 \%$ for the fitted total number of signal events.

The $1 \%$ agreement of energy scale between data and Monte Carlo is well within the $2-3 \%$ uncertainty on the energy scale transported from test-beam data analysis. The $1.5 \%$ discrepancy of the mass found in Monte Carlo with respect to the PDG nominal $\pi^{0}$ mass is consistent with the accuracy (as evaluated with simulation) of the cluster calibration procedure used for the low-energy photons, and the $1 \%$ uncertainty arising from the background modelling.

The converted photons reconstructed in section 6.7 can also be used to search for the $\pi^{0}$. This is done here using one photon reconstructed in the calorimeter, with a track veto applied and one conversion candidate. The conversion candidates are required to have four silicon hits on both tracks and must be in the same hemisphere as the calorimeter cluster. Figure 29(b) shows the $\gamma e^{+} e^{-}$mass spectrum; the $\pi^{0}$ peak is clearly visible. 


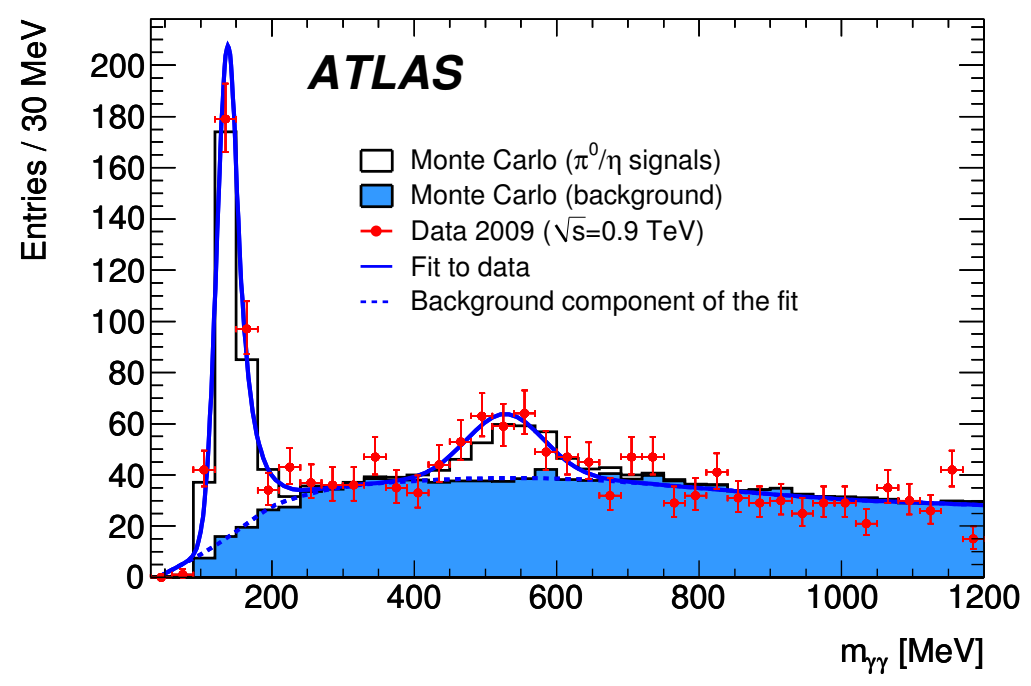

Figure 30. Diphoton invariant mass spectrum with tighter selection criteria to extract the $\eta$ peak with the fit superimposed to the data. The Monte Carlo simulation sample is normalized to the number of entries in the distribution for data.

The uniformity of the EM calorimeter response was studied in ten $\eta$ bins, where both photons are in the same bin. The diphoton mass distribution in each $\eta$ bin is fitted separately with the background shape constrained from simulation. The reconstructed $\pi^{0}$ mass is constant within 3\% for both data and Monte Carlo for all $\eta$ bins, and the ratio of data to Monte Carlo is consistent within the $2 \%$ statistical uncertainties.

\subsubsection{Extraction of the $\eta \rightarrow \gamma \gamma$ signal}

The number of $\eta \rightarrow \gamma \gamma$ events is expected to be one order of magnitude smaller than $\pi^{0} \rightarrow \gamma \gamma$ in the minimum-bias event sample. Therefore, the combinatorial background contribution in the $\eta$ mass region needs to be significantly reduced. This can be achieved by adding the following criteria to the $\pi^{0}$ analysis:

- Tighter kinematic selections: $\mathrm{E}_{T}^{\text {cluster }}>800 \mathrm{MeV}, \mathrm{p}_{T}^{\text {pair }}>2200 \mathrm{MeV}$.

- A track veto: no track, extrapolated into the calorimeter, should be within $-0.1<$ $\left(\phi_{\text {clus }}-\phi_{\text {extr }}\right)<0.05$ and $\left|\eta_{\text {clus }}-\eta_{\text {extr }}\right|<0.05$ of the cluster being considered.

The diphoton invariant mass spectrum of this sample is shown in figure 30 for both data and Monte Carlo. In addition to the $\pi^{0}$ peak, the $\eta \rightarrow \gamma \gamma$ signal can be observed on top of the combinatorial background. The mass spectrum was fitted using the sum of a Gaussian and a Crystal-Ball function with the same mean for the $\pi^{0}$ peak, a Gaussian for the $\eta$ peak and a $4^{\text {th }}$ order Chebyshev polynomial for the background. The $\pi^{0}$ and background shape parameters are taken from the Monte Carlo simulation while their normalizations are free in the fit, as are the parameters of the Gaussian describing the $\eta$ peak.

As can be seen from figure 30, the number of $\eta$ candidates per photon pair agrees between the data and the Monte Carlo simulation. The $\eta$ mass extracted from the data, 
$527 \pm 11$ (stat) $\mathrm{MeV}$, agrees with the mass obtained using the same fitting function on the Monte Carlo simulation, $544 \pm 3$ (stat) $\mathrm{MeV}$, within the statistical and energy scale uncertainties.

\section{Jets}

Many of the final states which will be studied in high energy collisions contain jets of hadrons produced by strong interactions. The ATLAS analysis chain applies the same jet algorithm to the $0.9 \mathrm{TeV}$ and $2.36 \mathrm{TeV}$ collision data and to the Monte Carlo simulation. The following comparison between data and Monte Carlo simulations should not be taken as a precise analysis of the underlying physics in the simulation, but rather as an assessment of the general behaviour of the detector and software chain (reconstruction and simulation).

Results are presented using clusters of calorimeter cells calibrated to correctly measure the energy deposited by electrons and photons in the calorimeter. This is known as the electromagnetic scale. There was, at this stage, no allowance for energy loss in inert material. From this starting point jets were reconstructed using the anti- $\mathrm{k}_{T}$ algorithm [19], which is safe against infrared and collinear divergences. The parameter $\mathrm{R}$, which controls the size of jets in the $\eta-\phi$ plane, was set to $\mathrm{R}=0.6$.

\subsection{Jets from calorimeter clusters}

The inputs to the jet algorithm are topological clusters [16] which attempt to reconstruct the three-dimensional shower topology of each particle. These clusters were built starting from seed cells with energies $\left|E_{i}\right|>4 \sigma_{\text {noise }}$, where $\sigma_{\text {noise }}$ is the electronic noise measured by iteratively gathering neighbouring cells with $\left|E_{j}\right|>2 \sigma_{\text {noise }}$ and, in a final step, adding all direct neighbours of these accumulated secondary cells. The noise in the EM calorimeter, for example, was in the range of 10-40 MeV per cell, depending upon the compartment and pseudorapidity. Approximately $0.1 \%$ of all cells were classified as noisy and were removed. Clusters built from the remaining cells were then used to create jets, which have to satisfy: $p_{\mathrm{T}}^{\text {jet }}>7 \mathrm{GeV}$ and $|\eta|<2.6$ where $p_{\mathrm{T}}^{\text {jet }}$ is the transverse jet momentum at the electromagnetic scale. In order to remove cosmic muons and some residual effects from cells in the calorimeter that exhibit large noise fluctuations the jets are required to pass quality criteria. Furthermore, if the jet energy corrections compensating for excluded calorimeter regions exceed $20 \%$, the corresponding candidates are not considered. Figure 31 presents example distributions of the internal structure of the jets, namely the number of topological clusters, and the fraction of the jet energy carried by each of them.

Figure 32 shows the jet $p_{\mathrm{T}}$ in data and Monte Carlo simulation, normalized to the number of jets in data. Figure 32(b) shows the difference of the azimuthal angle of the two leading jets $(\Delta \phi)$ in events with at least two reconstructed jets in data. The distribution peaks at $\pi$, corresponding to a topology where the two reconstructed jets are back-to-back in the transverse plane. In all distributions, the agreement between data and simulations is good, demonstrating that the description of the material and detector response in the simulation provides an adequate model of basic jet quantities. 

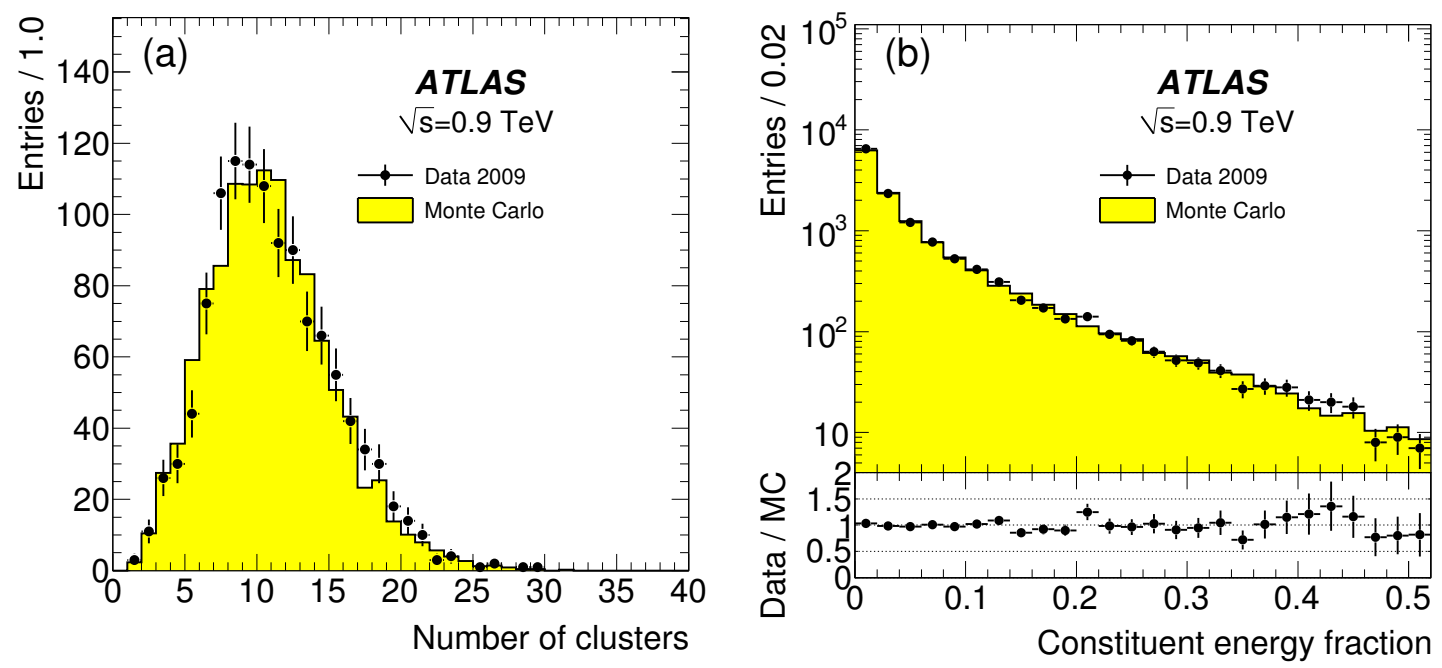

Figure 31. Distributions of (a) the number of clusters per jet and (b) the fraction of energy per cluster for jets reconstructed with topological clusters using the anti- $\mathrm{k}_{T}$ algorithm with $\mathrm{R}=0.6$.
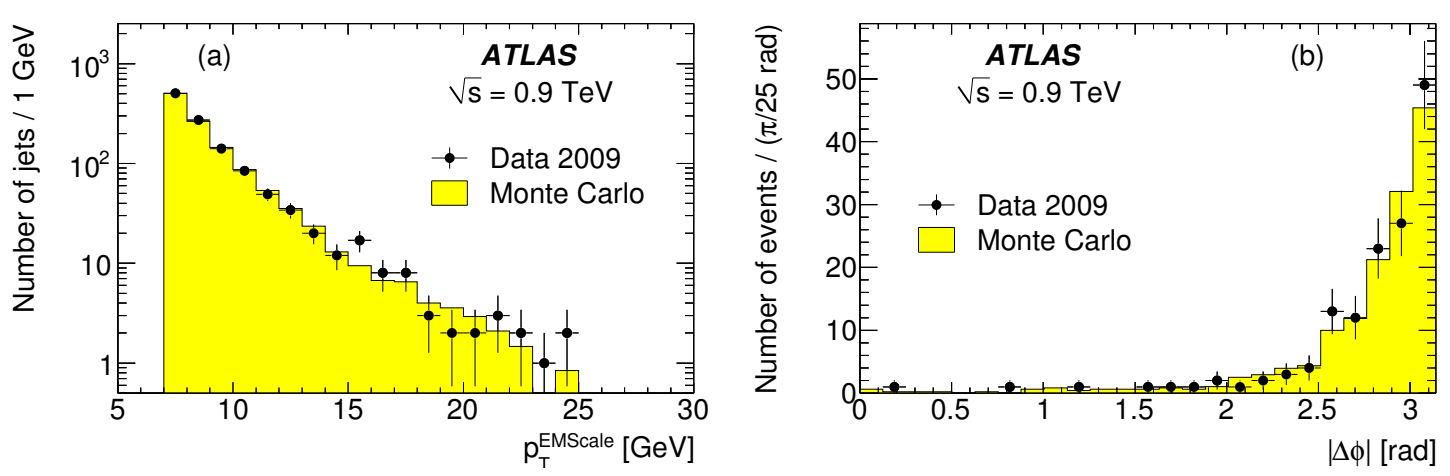

Figure 32. Distributions of (a) $p_{\mathrm{T}}$ for all jets and (b) $\Delta \phi$ for events with two or more jets. These are shown for jets reconstructed with topological clusters using the anti- $\mathrm{k}_{T}$ algorithm with $\mathrm{R}=0.6$.

\subsection{Performance of the first level jet trigger}

The efficiency of the L1 jet trigger has been studied. Jets were reconstructed as before, but with a lower energy threshold, $E_{\mathrm{T}}^{\text {jet }}>4 \mathrm{GeV}$. For the efficiency determination the jet signatures identified by the first level calorimeter trigger were matched to those of reconstructed jets by requiring $\Delta R<0.6$. L1 jet signatures are based on sums of trigger towers in the electromagnetic and hadronic calorimeter both calibrated to the electromagnetic scale. Trigger towers are formed by analogue summation on the detector and mostly have a size of $0.1 \times 0.1$ in $\eta \times \phi$, being larger in parts of the end-caps and in the FCal. For the lowest threshold jet trigger the trigger towers are summed over $0.4 \times 0.4$ in $\eta \times \phi$ and all higher threshold jet triggers use $0.8 \times 0.8$ in $\eta \times \phi$. The special treatment of the lowest threshold jet trigger is motivated by its higher susceptibility to noise.

The trigger efficiency is calculated as the fraction of reconstructed jets passing the 

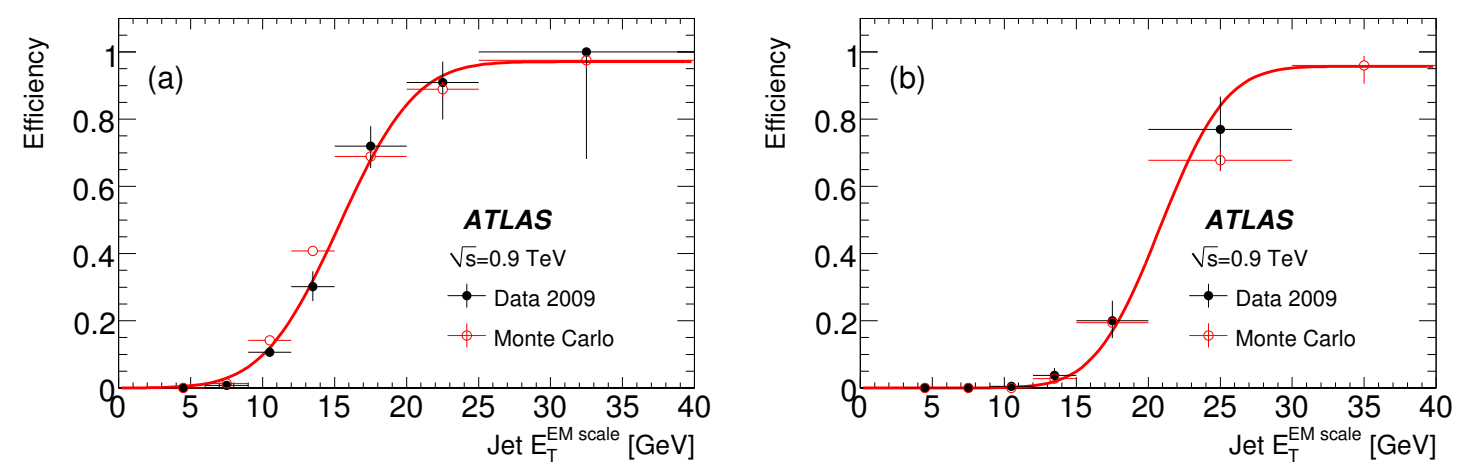

Figure 33. L1 jet trigger efficiency for the triggers with $50 \%$ efficiency at around $15 \mathrm{GeV}$ and $20 \mathrm{GeV}$ for data (solid) and simulation (open) together with a fit to the Monte Carlo using a standard threshold function (see text).
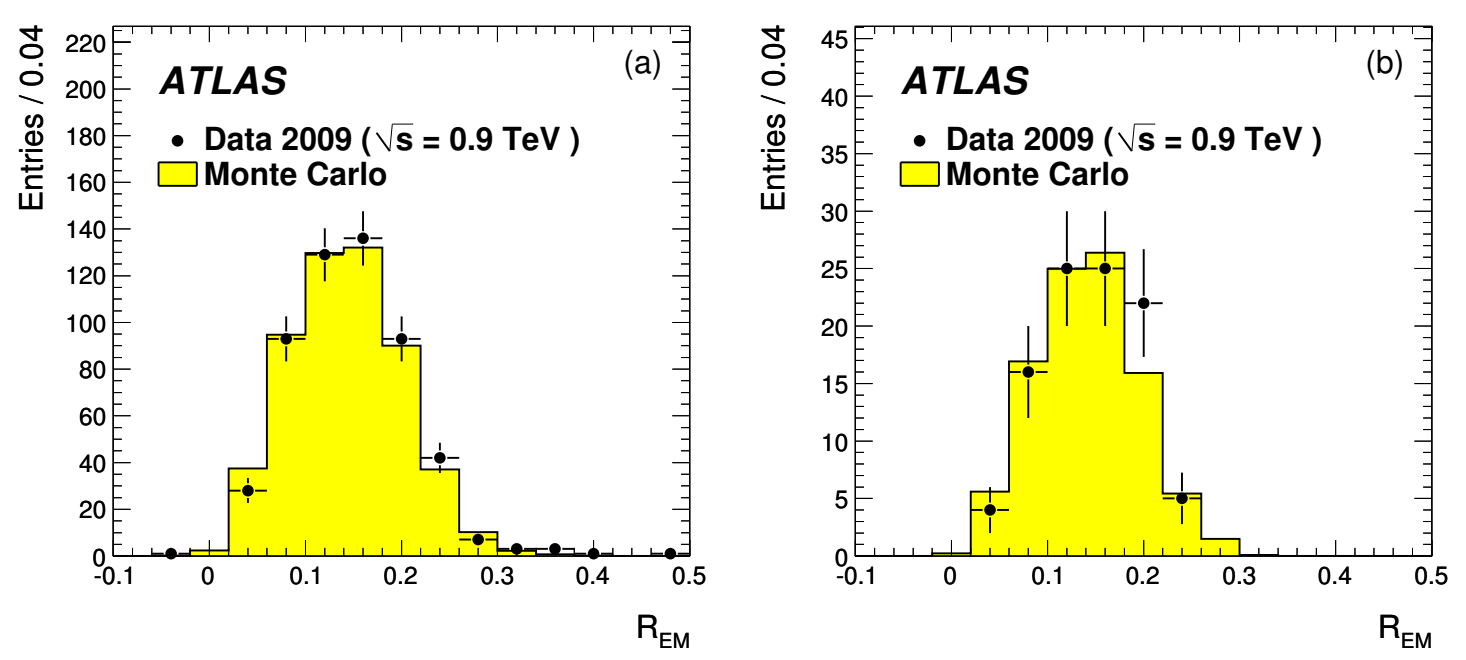

Figure 34. (a) The electromagnetic radius $R_{\mathrm{E} M}$ (see text) of the inclusive reconstructed tau candidates. (b) The same variable with a tightened selection requiring di-jet events. The Monte Carlo is normalized to the same number of candidates as in the data.

quality requirements described which have a matched trigger jet. The results for the two jet triggers with $50 \%$ efficiency at around $15 \mathrm{GeV}$ and $20 \mathrm{GeV}$ are shown for data and Monte Carlo simulation as a function of the reconstructed jet transverse energy, $E_{\mathrm{T}}^{\mathrm{jet}}$ in figure 33 , where $E_{\mathrm{T}}^{\text {jet }}$ is given at the electromagnetic scale. The curves are fits to the simulation using a standard trigger turn-on parameterization with an error function. The data are in agreement with the fit, which shows that the thresholds are as they are expected to be. This is a step to understanding the initial jet selection performance of the first-level calorimeter trigger.

\subsection{Tau studies using jets}

Jets from QCD processes form the largest background for the reconstruction of hadronically decaying tau candidates. Therefore, even though the actual number of tau leptons in the 
2009 data is expected to be negligible, basic track and cluster distributions have been studied using jets with emphasis on the variables of importance for tau reconstruction and identification. In figure 34 , the electromagnetic radius, $R_{\mathrm{E} M}$, (the energy-weighted mean $\Delta R$ of the jet components from the seed cell) is shown for all tau candidates with $E_{\mathrm{T}}^{\mathrm{jet}}>7 \mathrm{GeV}$ on the left and for a subset with a tight di-jet selection, which are more likely to be taken as $\tau$ candidates, on the right. Good agreement between data and simulation is observed, which gives confidence in the results obtained from earlier performance estimates based on Monte Carlo simulations.

\section{Missing transverse energy}

A reliable measurement of the missing transverse energy, $E_{\mathrm{T}}^{\text {miss }}$, is a key ingredient for many important analyses. This study considers $E_{\mathrm{T}}^{\text {miss }}$ reconstructed from calorimeter information only. As this measurement involves summing calorimeter cells over the whole detector, it is sensitive to detector and reconstruction effects. In particular events with rare unexpected noise contributions tend to appear in the tail of the $E_{\mathrm{T}}^{\text {miss }}$ spectrum.

$E_{\mathrm{T}}^{\text {miss }}$ reconstructed with the calorimeter is derived from the vector sum of the transverse energies of the cells. Because of the high granularity of the calorimeter (about 187000 cells), it is crucial to suppress noise contributions to $E_{\mathrm{T}}^{\text {miss }}$, i.e. to limit the number of cells used in the sum. This is done by only using cells belonging to three-dimensional topological clusters defined in section 7.1. About 800 and 2500 cells on average are included in such clusters in randomly triggered and collision events, respectively. The $0.1 \%$ of the cells classified as noisy are removed.

The sensitivity to noise can be best studied in randomly triggered events, where minimal energy is deposited in the calorimeters. The $E_{\mathrm{T}}^{\mathrm{miss}}$ distribution of these events is shown in figure 35, demonstrating the level of tails in randomly triggered events.

In soft proton-proton collisions, no true $E_{\mathrm{T}}^{\text {miss }}$ is expected. This is confirmed by the Monte Carlo simulation. Unlike in randomly triggered events, total transverse energies $\left(\sum E_{\mathrm{T}}\right)$ up to $100 \mathrm{GeV}$ are deposited in the calorimeter for minimum-bias events in the present data set. Figure 36 shows the measured $E_{\mathrm{y}}^{\text {miss }}$ distributions as an example. For both $E_{\mathrm{x}}^{\text {miss }}$ and $E_{\mathrm{y}}^{\text {miss }}$, the RMS values are about $1.4 \mathrm{GeV}$ and $1.8 \mathrm{GeV}$ for $0.9 \mathrm{TeV}$ and $2.36 \mathrm{TeV}$ centre-of-mass energies, respectively. These values are higher than in randomly triggered events because the finite resolution in the presence of real energy contributes to the width.

The $E_{\mathrm{T}}^{\text {miss }}$ distribution, also shown in figure 36 , is found to be satisfactory at this early stage. There are no events outside the borders of the figure. The two data events with large $E_{\mathrm{T}}^{\mathrm{miss}}$ are consistent with arising from out-of-time energy in the detector. At least one of these appears to be a cosmic ray event. Such events are not included in the Monte Carlo simulation sample.

A more quantitative evaluation of the $E_{\mathrm{T}}^{\text {miss }}$ performance can be obtained from a study of the $E_{\mathrm{x}}^{\mathrm{miss}}$ and $E_{\mathrm{y}}^{\mathrm{miss}}$ resolutions as a function of the total transverse energy $\sum E_{\mathrm{T}}$ in the event. The resolutions are expected to be proportional to $\sqrt{\sum E_{\mathrm{T}}}$. The resolutions observed in the ATLAS data at both centre-of-mass energies are presented as a function 


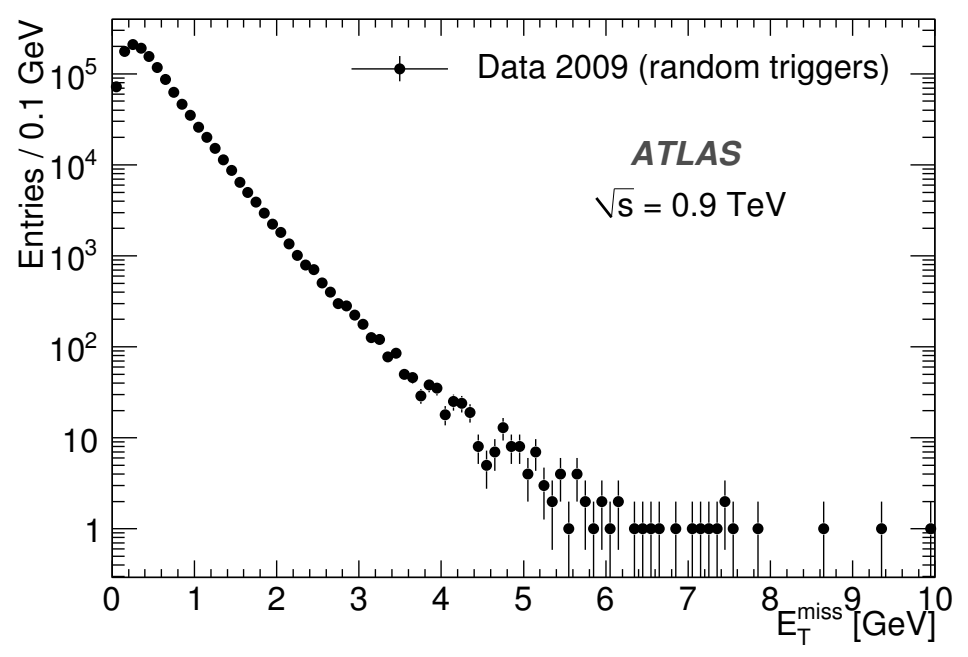

Figure 35. Distribution of $E_{\mathrm{T}}^{\mathrm{miss}}$ as measured in data from randomly triggered events. Only cells belonging to topological clusters are included in the calculation; their energies are calibrated at the EM scale.

of $\sqrt{\sum E_{\mathrm{T}}}$ in figure 37. A very good agreement between data and Monte Carlo simulation is obtained at both centre-of-mass energies. The $E_{\mathrm{T}}^{\text {miss }}$ resolution can be parameterized as $\sigma\left(E_{\mathrm{x}}^{\text {miss }}, E_{\mathrm{y}}^{\text {miss }}\right)=0.37 \times \sqrt{\sum E_{\mathrm{T}}}$ at the EM scale, ${ }^{2}$ with a negligible statistical error.

\section{Muons}

The calorimeters are surrounded by the muon spectrometer [4], which was designed to provide a trigger and accurate standalone reconstruction for muons with $p_{\mathrm{T}}$ from several $\mathrm{GeV}$ up to a few $\mathrm{TeV}$. In contrast, the muons studied in the sample collected at $\sqrt{s}=0.9 \mathrm{TeV}$ are of relatively low $p_{\mathrm{T}}$. The air-core toroid system, with a long barrel and two inserted end-cap magnets, generates an average field of $0.5 \mathrm{~T}(1 \mathrm{~T})$, in the barrel (end-caps) over a large volume. Multiple scattering effects are thereby minimized, and excellent muon momentum resolution is achieved with three layers of high precision tracking chambers. Over most of the $\eta$ range, tracks are measured by Monitored Drift Tubes (MDT). For $2<$ $|\eta|<2.7$, Cathode Strip Chambers (CSC) with higher granularity are used in the innermost layer, to withstand the demanding rate and background conditions. In addition, the muon spectrometer includes dedicated trigger chambers with nominal timing resolutions between 1.5 and 4 ns. They are composed of Resistive Plate Chambers (RPC) in the barrel and Thin-Gap Chambers (TGC) in the end-cap regions. Besides providing trigger signals they also measure the muon coordinate in the direction orthogonal to that determined by the precision-tracking chambers.

The muon analysis uses a somewhat smaller data set than other analyses as the toroid system was not always operational. In addition, it was checked that the MDTs, TGCs

\footnotetext{
${ }^{2} \sigma\left(E_{\mathrm{x}}^{\mathrm{miss}}, E_{\mathrm{y}}^{\mathrm{miss}}\right)$ and $E_{\mathrm{T}}$ in $\mathrm{GeV}$.
} 

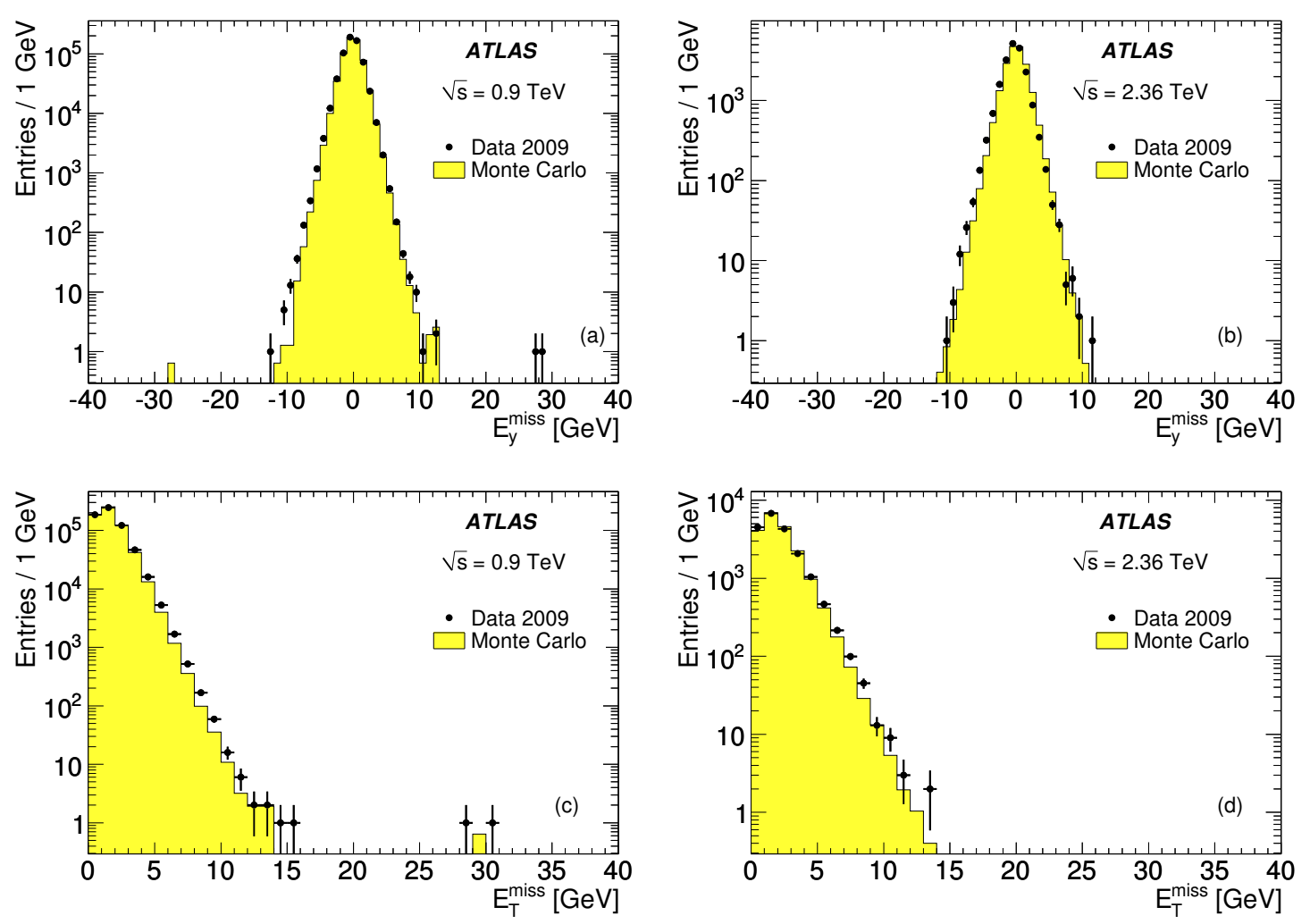

Figure 36. Distribution of $E_{\mathrm{y}}^{\text {miss }}(\mathrm{a}, \mathrm{b})$ and $E_{\mathrm{T}}^{\mathrm{miss}}(\mathrm{c}, \mathrm{d})$ as measured in data from minimum-bias events (dots) at $0.9 \mathrm{TeV}(\mathrm{a}, \mathrm{c})$ and $2.36 \mathrm{TeV}(\mathrm{b}, \mathrm{d})$ centre-of-mass energies. In the calculation only topological cluster cells are used, with energies calibrated at the EM scale. The expectations from Monte Carlo simulation are superimposed (histograms) and normalized to the number of events in data.

and RPCs were all operating normally. The algorithms used for muon reconstruction combine tracks from the muon systems and the inner detector, and are developed from those described in ref. [16]. For the results presented here, two independent algorithms are used and only candidates selected by both are accepted. This selects a total of 50 muon candidates. Raw kinematic distributions for these candidates are presented in figure 38. The muon spectrum observed is soft and strongly peaked in the forward direction, where the momentum of the muons more often exceeds the $3.2 \mathrm{GeV}$ needed to penetrate through the forward calorimeter. The kinematic distributions are compared to the predictions from minimum-bias simulation with the Monte Carlo normalized to the number of muons found in data. Within the large statistical uncertainties good agreement is found indicating a reasonable understanding of the initial performance of the ATLAS muon spectrometer.

The muon trigger, designed to select high- $p_{\mathrm{T}}$ muons, has a limited acceptance for the muon tracks reconstructed offline in 2009. Of the 38 muons in the end-cap regions, 13 were triggered at L1 by the TGC. Of the 12 muons in the barrel, one was triggered at L1 by the RPC with the correct timing, another 9 were triggered with +1 bunch crossing misalignment during the timing adjustment phase, while the other 2 muons were outside the 


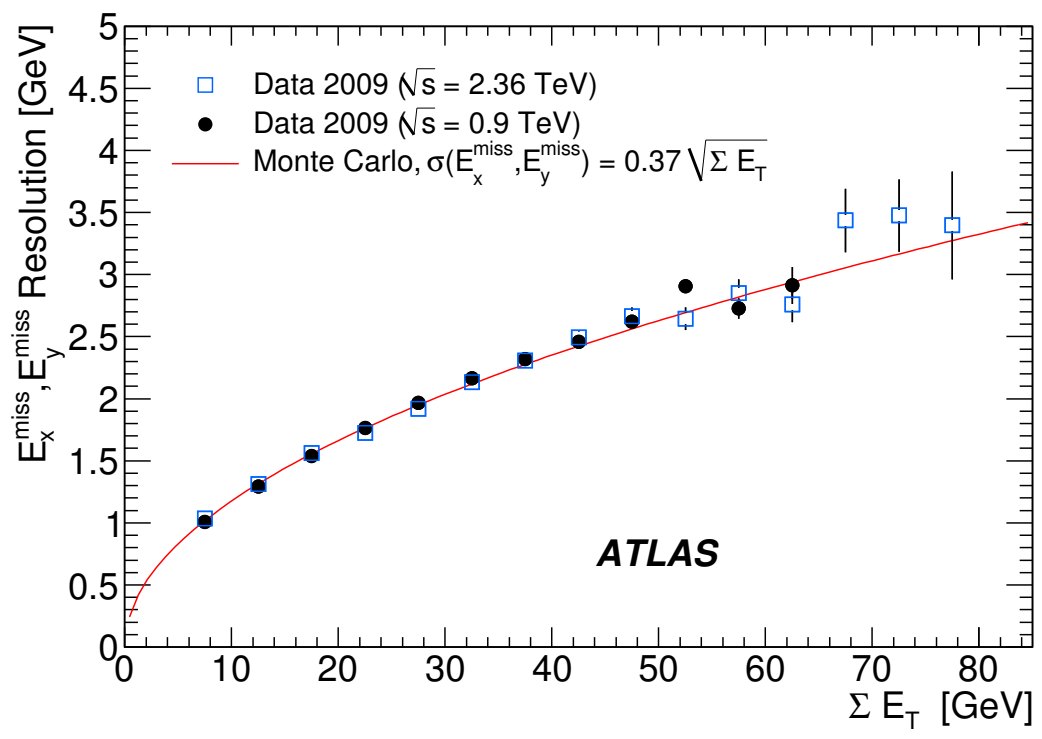

Figure 37. $E_{\mathrm{T}}^{\text {miss }}$ resolution as a function of the total transverse energy $\left(\sum E_{\mathrm{T}}\right)$ for minimum-bias events. The line shows a fit to the resolution expected from the Monte Carlo simulation and the full dots (open squares) represents the results with data at $0.9(2.36) \mathrm{TeV} . E_{\mathrm{x}}^{\text {miss }}, E_{\mathrm{y}}^{\text {miss }}, \sum E_{\mathrm{T}}$ are computed with topological cluster cells at EM scale.
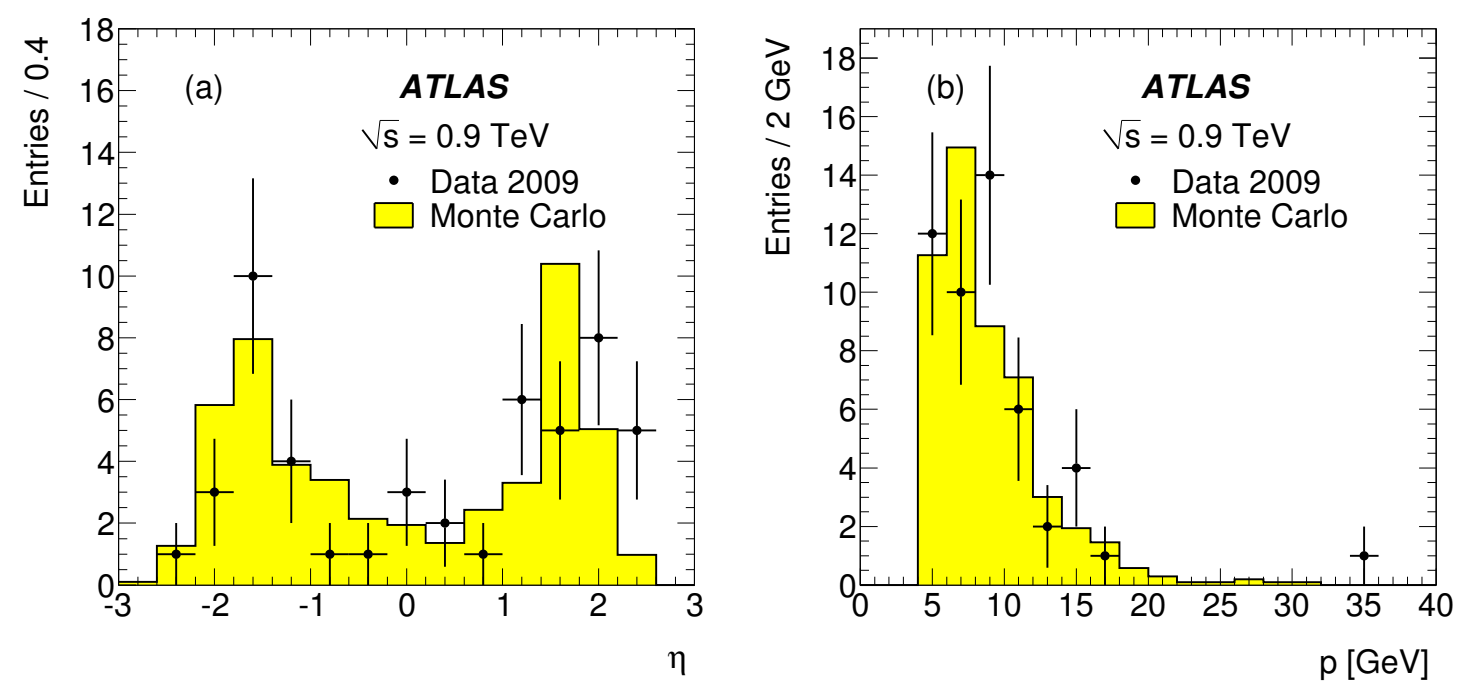

Figure 38. The distribution of $\eta$ and $p$ of reconstructed muons in the $0.9 \mathrm{TeV}$ data. The simulation distributions are normalized to the number of entries in data.

trigger acceptance. Only one muon passed the full trigger chain up to the EF combined trigger after applying the $p_{\mathrm{T}}>4 \mathrm{GeV}$ selection. The muon momenta and directions measured by the L2 and EF are in good agreement with the offline measurement. 


\section{Conclusions}

The overall performance of the ATLAS detector at the LHC was established in first collision data at centre-of-mass energies of $0.9 \mathrm{TeV}$ and $2.36 \mathrm{TeV}$. Although the detector has not been optimized for the low energy particles studied, its performance was found to be remarkably good, particularly in view of the early stage of data taking.

The overall data-taking efficiency was about $90 \%$ and the subdetectors were typically 99\% operational. The entire computing infrastructure of Trigger/DAQ was immediately effective. Collision candidates were selected with a negligible background level.

The tracking detectors and electromagnetic calorimeters have, by the nature of the data set, been the most extensively tested components and they perform well. The hit efficiencies, resolutions and particle identification capabilities of the tracking detector are well modelled by Monte Carlo simulations. The discrimination between electrons and pions using transition radiation was demonstrated and outperforms any previous colliding beam experiment. The results of various studies suggest that the material distribution in the inner part of the tracker is well understood, at a level of a few percent of the total in the barrel silicon systems.

The momentum scale linked to the $K_{\mathrm{S}}^{0}$ mass is known at the per mille level and the calibration of the electromagnetic calorimetry in the region of the $\pi^{0}$ mass was checked at the level of $1 \%$. Electron and photon reconstruction was extensively tested and performs well. The candidates typically have transverse momenta well below those for which reconstruction and identification algorithms were optimized, but their properties are shown to be in good agreement with simulation. Good calorimeter performance was also demonstrated by the measurement of the resolution of the missing transverse energy, which follows closely the expectations from Monte Carlo over the entire energy range probed.

The muon system was not extensively tested with this data set, but performs as expected within the precision available. It was well tested in cosmic ray data taken in 2008 [4].

\section{Acknowledgments}

We are greatly indebted to all CERN's departments and to the LHC project for their immense efforts not only in building the LHC, but also for their direct contributions to the construction and installation of the ATLAS detector and its infrastructure. All our congratulations go to the LHC operation team for the superb performance during this initial data-taking period. We acknowledge equally warmly all our technical colleagues in the collaborating Institutions without whom the ATLAS detector could not have been built. Furthermore we are grateful to all the funding agencies which supported generously the construction and the commissioning of the ATLAS detector and also provided the computing infrastructure.

The ATLAS detector design and construction has taken about fifteen years, and our thoughts are with all our colleagues who sadly could not see its final realisation.

We acknowledge the support of ANPCyT, Argentina; Yerevan Physics Institute, Armenia; ARC and DEST, Australia; Bundesministerium für Wissenschaft und Forschung, 
Austria; National Academy of Sciences of Azerbaijan; State Committee on Science \& Technologies of the Republic of Belarus; CNPq and FINEP, Brazil; NSERC, NRC, and CFI, Canada; CERN; CONICYT, Chile; NSFC, China; COLCIENCIAS, Colombia; Ministry of Education, Youth and Sports of the Czech Republic, Ministry of Industry and Trade of the Czech Republic, and Committee for Collaboration of the Czech Republic with CERN; Danish Natural Science Research Council and the Lundbeck Foundation; European Commission, through the ARTEMIS Research Training Network; IN2P3-CNRS and DapniaCEA, France; Georgian Academy of Sciences; BMBF, DFG, HGF and MPG, Germany; Ministry of Education and Religion, through the EPEAEK program PYTHAGORAS II and GSRT, Greece; ISF, MINERVA, GIF, DIP, and Benoziyo Center, Israel; INFN, Italy; MEXT, Japan; CNRST, Morocco; FOM and NWO, Netherlands; The Research Council of Norway; Ministry of Science and Higher Education, Poland; GRICES and FCT, Portugal; Ministry of Education and Research, Romania; Ministry of Education and Science of the Russian Federation and State Atomic Energy Corporation ROSATOM; JINR; Ministry of Science, Serbia; Department of International Science and Technology Cooperation, Ministry of Education of the Slovak Republic; Slovenian Research Agency, Ministry of Higher Education, Science and Technology, Slovenia; Ministerio de Educación y Ciencia, Spain; The Swedish Research Council, The Knut and Alice Wallenberg Foundation, Sweden; State Secretariat for Education and Science, Swiss National Science Foundation, and Cantons of Bern and Geneva, Switzerland; National Science Council, Taiwan; TAEK, Turkey; The Science and Technology Facilities Council and The Leverhulme Trust, United Kingdom; DOE and NSF, United States of America.

Open Access. This article is distributed under the terms of Creative Commence Attribution License (CC-BY-3.0), which permits any use provided the original author(s) and source are credited. This work shall be referred to as: ATLAS Collaboration, Performance of the ATLAS detector using first collision data, JHEP09(2010)056, doi: 10.1007/JHEP09(2010)056.

\section{References}

[1] ATLAS collaboration, G. Aad et al., The ATLAS Experiment at the CERN Large Hadron Collider, 2008 JINST 3 S08003 [SPIRES].

[2] ATLAS collaboration, G. Aad et al., Charged-particle multiplicities in pp interactions at $\sqrt{s}$ $=900 \mathrm{GeV}$ measured with the ATLAS detector at the LHC, Phys. Lett. B 688 (2010) 21 [arXiv: 1003.3124] [SPIRES].

[3] ATLAS collaboration, G. Aad et al., Readiness of the ATLAS Liquid Argon Calorimeter for LHC Collisions, arXiv:0912.2642 [SPIRES].

[4] ATLAS collaboration, Commissioning of the ATLAS Muon Spectrometer with Cosmic Rays, submitted to Eur. Phys. J. C [arXiv:1006.4384] [SPIRES].

[5] ATLAS collaboration, P. Ryan et al., The ATLAS Inner Detector commissioning and calibration, accepted by Eur. Phys. J. C [arXiv:1004.5293] [SPIRES].

[6] ATLAS collaboration, G. Aad et al., Drift Time Measurement in the ATLAS Liquid Argon Electromagnetic Calorimeter using Cosmic Muons, arXiv:1002.4189 [SPIRES]. 
[7] T. Sjöstrand, S. Mrenna and P.Z. Skands, PYTHIA 6.4 Physics and Manual, JHEP 05 (2006) 026 [hep-ph/0603175] [SPIRES].

[8] G. Aad et al., ATLAS Monte Carlo tunes for MC09, ATLAS physics note ATL-PHYS-PUB-2010-002 (2010).

[9] A.D. Martin, R.G. Roberts, W.J. Stirling and R.S. Thorne, Parton distributions incorporating QED contributions, Eur. Phys. J. C 39 (2005) 155 [hep-ph/0411040] [SPIRES].

[10] ATLAS collaboration and others, The ATLAS Simulation Infrastructure, arXiv: 1005.4568 [SPIRES].

[11] GEANT4 collaboration, S. Agostinelli et al., GEANT4: A simulation toolkit, Nucl. Instrum. Meth. A 506 (2003) 250 [SPIRES].

[12] P. Adragna et al., Measurement of pion and proton response and longitudinal shower profiles up to 20 nuclear interaction lengths with the ATLAS tile calorimeter, Nucl. Instrum. Meth. A 615 (2010) 158 [SPIRES].

[13] T. Cornelisson et al., The New ATLAS Track Reconstruction (NEWT), J. Phys. Conf. Ser. 119 (2008) 032014.

[14] Particle Data Group collaboration, C. Amsler et al., Review of particle physics, Phys. Lett. B 667 (2008) 1 [SPIRES].

[15] M. Aleksa et al., Measurement of the ATLAS solenoid magnetic field, 2008 JINST 3 P04003 [SPIRES].

[16] The ATLAS collaboration, G. Aad et al., Expected Performance of the ATLAS Experiment, Detector, Trigger and Physics, CERN-OPEN-2008-020 (2008).

[17] J. Colas et al., Response Uniformity of the ATLAS Liquid Argon Electromagnetic Calorimeter, Nucl. Instrum. Meth. A 582 (2007) 429 [arXiv:0709.1094] [SPIRES].

[18] The Crystal Ball collaboration, M.J. Oreglia, A study of the reaction $\psi \prime \rightarrow \gamma \gamma \psi$, SLAC-R-236 (1980) [SPIRES].

[19] M. Cacciari, G.P. Salam and G. Soyez, The anti-kt jet clustering algorithm, JHEP 04 (2008) 063 [arXiv: 0802.1189] [SPIRES]. 


\section{The ATLAS collaboration}

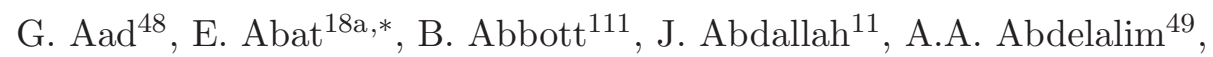

A. Abdesselam ${ }^{118}$, O. Abdinov ${ }^{10}$, B. Abi ${ }^{112}$, M. Abolins ${ }^{88}$, H. Abramowicz ${ }^{152}$,

H. Abreu ${ }^{115}$, E. Acerbi ${ }^{89 a, 89 b}$, B.S. Acharya ${ }^{163 a, 163 b}$, M. Ackers ${ }^{20}$, D.L. Adams ${ }^{24}$, T.N. Addy ${ }^{56}$, J. Adelman ${ }^{174}$, M. Aderholz ${ }^{99}$, S. Adomeit ${ }^{98}$, C. Adorisio ${ }^{36 a, 36 b}$, P. Adragna ${ }^{75}$, T. Adye ${ }^{129}$, S. Aefsky ${ }^{22}$, J.A. Aguilar-Saavedra ${ }^{124 b}$, M. Aharrouche ${ }^{81}$, S.P. Ahlen ${ }^{21}$, F. Ahles ${ }^{48}$, A. Ahmad ${ }^{147}$, H. Ahmed ${ }^{2}$, M. Ahsan ${ }^{40}$, G. Aielli ${ }^{133 a, 133 b}$, T. Akdogan ${ }^{18 a}$, P.F. Åkesson ${ }^{29}$, T.P.A. Åkesson ${ }^{79}$, G. Akimoto ${ }^{154}$, A.V. Akimov ${ }^{94}$,

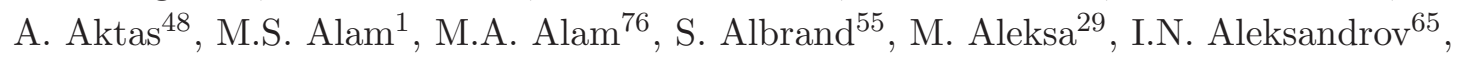
M. Aleppo ${ }^{89 a, 89 b}$, F. Alessandria ${ }^{89 a}$, C. Alexa ${ }^{25 a}$, G. Alexander ${ }^{152}$, G. Alexandre ${ }^{49}$,

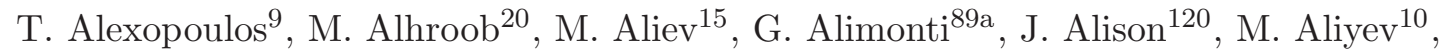
P.P. Allport ${ }^{73}$, S.E. Allwood-Spiers ${ }^{53}$, J. Almond ${ }^{82}$, A. Aloisio ${ }^{102 a, 102 b}$, R. Alon ${ }^{170}$,

A. Alonso ${ }^{79}$, J. Alonso ${ }^{14}$, M.G. Alviggi ${ }^{102 a, 102 b}$, K. Amako ${ }^{66}$, P. Amaral ${ }^{29}$,

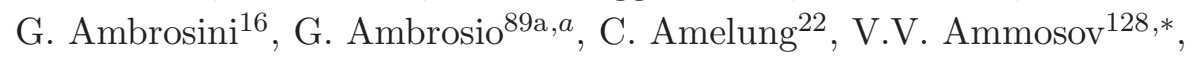

V.V. Ammosov ${ }^{128, *}$, A. Amorim ${ }^{124 a}$, G. Amorós ${ }^{166}$, N. Amram ${ }^{152}$, C. Anastopoulos ${ }^{139}$, T. Andeen ${ }^{29}$, C.F. Anders ${ }^{48}$, K.J. Anderson ${ }^{30}$, A. Andreazza ${ }^{89 a, 89 b}$, V. Andrei ${ }^{58 a}$, M-L. Andrieux ${ }^{55}$, X.S. Anduaga ${ }^{70}$, A. Angerami ${ }^{34}$, F. Anghinolfi $^{29}$, N. Anjos ${ }^{124 a}$, A. Annovi ${ }^{47}$, A. Antonaki ${ }^{8}$, M. Antonelli ${ }^{47}$, S. Antonelli ${ }^{19 a, 19 b}, J^{2}$ Antos $^{144 b}$, B. Antunovic ${ }^{41}$, F. Anulli ${ }^{132 a}$, S. Aoun ${ }^{83}$, G. Arabidze ${ }^{8}$, I. Aracena ${ }^{143}$, Y. Arai ${ }^{66}$, A.T.H. Arce ${ }^{14}$, J.P. Archambault ${ }^{28}$, S. Arfaoui ${ }^{29, b}$, J-F. Arguin ${ }^{14}$, T. Argyropoulos ${ }^{9}$, E. Arik ${ }^{18 a, *}$, M. Arik ${ }^{18 a}$, A.J. Armbruster ${ }^{87}$, K.E. Arms ${ }^{109}$, S.R. Armstrong ${ }^{24}$, O. Arnaez ${ }^{4}$, C. Arnault ${ }^{115}$, A. Artamonov ${ }^{95}$, D. Arutinov ${ }^{20}$, M. Asai ${ }^{143}$, S. Asai ${ }^{154}$, R. Asfandiyarov ${ }^{171}$, S. Ask ${ }^{82}$, B. Åsman ${ }^{145 a, 145 b}$, D. Asner ${ }^{28}$, L. Asquith ${ }^{77}$, K. Assamagan ${ }^{24}$, A. Astbury ${ }^{168}$, A. Astvatsatourov ${ }^{52}$, G. Atoian ${ }^{174}$, B. Aubert ${ }^{4}$, B. Auerbach ${ }^{174}$, E. Auge ${ }^{115}$, K. Augsten ${ }^{127}$, M. Aurousseau ${ }^{4}$, N. Austin ${ }^{73}$, G. Avolio ${ }^{162}$, R. Avramidou ${ }^{9}$, D. Axen ${ }^{167}$, C. Ay ${ }^{54}$, G. Azuelos ${ }^{93, c}$, Y. Azuma ${ }^{154}$, M.A. Baak ${ }^{29}$, G. Baccaglioni ${ }^{89 a}$, C. Bacci ${ }^{134 a, 134 b}$, A.M. Bach ${ }^{14}$, H. Bachacou ${ }^{136}$, K. Bachas ${ }^{29}$, G. Bachy ${ }^{29}$, M. Backes ${ }^{49}$, E. Badescu ${ }^{25 a}$, P. Bagnaia ${ }^{132 a, 132 b}$, Y. Bai ${ }^{32 a}$, D.C. Bailey ${ }^{157}$, T. Bain ${ }^{157}$, J.T. Baines ${ }^{129}$, O.K. Baker ${ }^{174}$, M.D. Baker ${ }^{24}$, S Baker ${ }^{77}$,

F. Baltasar Dos Santos Pedrosa ${ }^{29}$, E. Banas ${ }^{38}$, P. Banerjee ${ }^{93}$, S. Banerjee ${ }^{168}$, D. Banfi ${ }^{89 a, 89 b}$, A. Bangert ${ }^{137}$, V. Bansal ${ }^{168}$, S.P. Baranov ${ }^{94}$, S. Baranov ${ }^{65}$,

A. Barashkou ${ }^{65}$, T. Barber ${ }^{27}$, E.L. Barberio ${ }^{86}$, D. Barberis ${ }^{50 a, 50 b}$, M. Barbero ${ }^{20}$, D.Y. Bardin ${ }^{65}$, T. Barillari ${ }^{99}$, M. Barisonzi ${ }^{173}$, T. Barklow ${ }^{143}$, N. Barlow ${ }^{27}$, B.M. Barnett ${ }^{129}$, R.M. Barnett ${ }^{14}$, A. Baroncelli ${ }^{134 a}$, M. Barone ${ }^{47}$, A.J. Barr ${ }^{118}$, F. Barreiro ${ }^{80}$, J. Barreiro Guimarães da Costa $^{57}$, P. Barrillon ${ }^{115}$, R. Bartoldus ${ }^{143}$, D. Bartsch ${ }^{20}$, R.L. Bates ${ }^{53}$, L. Batkova ${ }^{144 a}$, J.R. Batley ${ }^{27}$, A. Battaglia ${ }^{16}$, M. Battistin ${ }^{29}$, G. Battistoni ${ }^{89 a}$, F. Bauer ${ }^{136}$, H.S. Bawa ${ }^{143}$, M. Bazalova ${ }^{125}$, B. Beare ${ }^{157}$, T. Beau ${ }^{78}$, P.H. Beauchemin ${ }^{118}$, R. Beccherle ${ }^{50 a}$, N. Becerici ${ }^{18 a}$, P. Bechtle ${ }^{41}$, G.A. Beck ${ }^{75}$, H.P. Beck ${ }^{16}$, M. Beckingham ${ }^{48}$, K.H. Becks ${ }^{173}$, A.J. Beddall ${ }^{18 c}$, A. Beddall ${ }^{18 c}$, V.A. Bednyakov ${ }^{65}$, C. Bee ${ }^{83}$, M. Begel ${ }^{24}$, S. Behar Harpaz ${ }^{151}$, P.K. Behera ${ }^{63}$, M. Beimforde ${ }^{99}$, G.A.N. Belanger ${ }^{28}$, C. Belanger-Champagne ${ }^{165}$, B. Belhorma ${ }^{55}$, P.J. Bell ${ }^{49}$, W.H. Bell ${ }^{49}$, G. Bella ${ }^{152}$, L. Bellagamba ${ }^{19 a}$, F. Bellina $^{29}$, G. Bellomo ${ }^{89 a, 89 b}$, 
M. Bellomo ${ }^{119 a}$, A. Belloni ${ }^{57}$, K. Belotskiy ${ }^{96}$, O. Beltramello ${ }^{29}$, A. Belymam ${ }^{75}$, S. Ben Ami ${ }^{151}$, O. Benary ${ }^{152}$, D. Benchekroun ${ }^{135 a}$, C. Benchouk ${ }^{83}$, M. Bendel ${ }^{81}$, B.H. Benedict ${ }^{162}$, N. Benekos ${ }^{164}$, Y. Benhammou ${ }^{152}$, G.P. Benincasa ${ }^{124 a}$, D.P. Benjamin ${ }^{44}$, M. Benoit ${ }^{115}$, J.R. Bensinger ${ }^{22}$, K. Benslama ${ }^{130}$, S. Bentvelsen ${ }^{105}$, M. Beretta ${ }^{47}$, D. Berge ${ }^{29}$, E. Bergeaas Kuutmann ${ }^{41}$, N. Berger ${ }^{4}$, F. Berghaus ${ }^{168}$, E. Berglund ${ }^{49}$, J. Beringer ${ }^{14}$, K. Bernardet ${ }^{83}$, P. Bernat ${ }^{115}$, R. Bernhard ${ }^{48}$, C. Bernius ${ }^{77}$, T. Berry ${ }^{76}$, A. Bertin ${ }^{19 a, 19 b}$, F. Bertinelli29 ${ }^{29}$ S. Bertolucci ${ }^{47}$, M.I. Besana ${ }^{89 a, 89 b}$, N. Besson ${ }^{136}$, S. Bethke ${ }^{99}$, R.M. Bianchi ${ }^{48}$, M. Bianco ${ }^{72 a, 72 b}$, O. Biebel ${ }^{98}$, M. Bieri $^{142}$, J. Biesiada ${ }^{14}$, M. Biglietti132a,132b, H. Bilokon ${ }^{47}$, M. Binder ${ }^{98}$, M. Bindi ${ }^{19 a, 19 b}$, S. Binet ${ }^{115}$, A. Bingul ${ }^{18 c}$, C. Bini ${ }^{132 a, 132 b}$, C. Biscarat ${ }^{179}$, R. Bischof ${ }^{62}$, U. Bitenc ${ }^{48}$, K.M. Black ${ }^{57}$, R.E. Blair ${ }^{5}$, O. Blanch ${ }^{11}$, J-B Blanchard ${ }^{115}$, G. Blanchot ${ }^{29}$, C. Blocker ${ }^{22}$, J. Blocki ${ }^{38}$, A. Blondel ${ }^{49}$, W. Blum ${ }^{81}$, U. Blumenschein ${ }^{54}$, C. Boaretto ${ }^{132 a, 132 b}$, G.J. Bobbink ${ }^{105}$, A. Bocci ${ }^{44}$, D. Bocian ${ }^{38}$, R. Bock ${ }^{29}$, M. Boehler ${ }^{41}$, J. Boek ${ }^{173}$, N. Boelaert ${ }^{79}$, S. Böser ${ }^{77}$, J.A. Bogaerts ${ }^{29}$, A. Bogouch ${ }^{90, *}$, C. Bohm ${ }^{145 a}$, J. Bohm ${ }^{125}$, V. Boisvert ${ }^{76}$, T. Bold ${ }^{162, d}$, V. Boldea ${ }^{25 a}$, V.G. Bondarenko ${ }^{96}$, M. Bondioli $^{162}$, R. Bonino ${ }^{49}$, M. Boonekamp ${ }^{136}$, G. Boorman ${ }^{76}$, M. Boosten ${ }^{29}$, C.N. Booth ${ }^{139}$, P.S.L. Booth ${ }^{73, *}$, P. Booth ${ }^{139}$, J.R.A. Booth ${ }^{17}$, S. Bordoni ${ }^{78}$, C. Borer ${ }^{16}$, K. Borer ${ }^{16}$, A. Borisov ${ }^{128}$, G. Borissov ${ }^{71}$, I. Borjanovic ${ }^{12 a}$, S. Borroni $^{132 a, 132 b}$, K. Bos $^{105}$, D. Boscherini ${ }^{19 a}$, M. Bosman ${ }^{11}$, H. Boterenbrood ${ }^{105}$, D. Botterill ${ }^{129}$, J. Bouchami ${ }^{93}$, J. Boudreau ${ }^{123}$, E.V. Bouhova-Thacker ${ }^{71}$, C. Boulahouache ${ }^{123}$, C. Bourdarios ${ }^{115}$, A. Boveia ${ }^{30}$, J. Boyd ${ }^{29}$, I.R. Boyko ${ }^{65}$, N.I. Bozhko ${ }^{128}$, I. Bozovic-Jelisavcic ${ }^{12 b}$, S. Braccini ${ }^{47}$, J. Bracinik ${ }^{17}$, A. Braem ${ }^{29}$, E. Brambilla ${ }^{72 a, 72 b}$, P. Branchini ${ }^{134 a}$, G.W. Brandenburg ${ }^{57}$, A. Brandt ${ }^{7}$, G. Brandt ${ }^{41}$, O. Brandt ${ }^{54}$, U. Bratzler ${ }^{155}$, B. Brau ${ }^{84}$, J.E. Brau ${ }^{114}$, H.M. Braun ${ }^{173}$, S. Bravo ${ }^{11}$, B. Brelier ${ }^{157}$, J. Bremer ${ }^{29}$, R. Brenner ${ }^{165}$, S. Bressler ${ }^{151}$, D. Breton ${ }^{115}$, N.D. Brett ${ }^{118}$, P.G. Bright-Thomas ${ }^{17}$, D. Britton ${ }^{53}$, F.M. Brochu ${ }^{27}$, I. Brock ${ }^{20}$, R. Brock ${ }^{88}$, T.J. Brodbeck ${ }^{71}$, E. Brodet ${ }^{152}$, F. Broggi ${ }^{89 a}$, C. Bromberg ${ }^{88}$, G. Brooijmans ${ }^{34}$, W.K. Brooks ${ }^{31 b}$, G. Brown ${ }^{82}$, E. Brubaker ${ }^{30}$, P.A. Bruckman de Renstrom ${ }^{38}$, D. Bruncko ${ }^{144 b}$, R. Bruneliere ${ }^{48}$, S. Brunet ${ }^{41}$, A. Bruni ${ }^{19 a}$, G. Bruni ${ }^{19 a}$, M. Bruschi ${ }^{19 a}$, T. Buanes ${ }^{13}$, F. Bucci ${ }^{49}$, J. Buchanan ${ }^{118}$, N.J. Buchanan ${ }^{2}$, P. Buchholz ${ }^{141}$, A.G. Buckley ${ }^{45}$, I.A. Budagov ${ }^{65}$, B. Budick ${ }^{108}$, V. Büscher ${ }^{81}$, L. Bugge ${ }^{117}$, D. Buira-Clark ${ }^{118}$, E.J. Buis ${ }^{105}$, F. Bujor ${ }^{29}$, O. Bulekov ${ }^{96}$, M. Bunse ${ }^{42}$, T. Buran ${ }^{117}$, H. Burckhart ${ }^{29}$, S. Burdin ${ }^{73}$, T. Burgess ${ }^{13}$, S. Burke ${ }^{129}$, E. Busato ${ }^{33}$, P. Bussey ${ }^{53}$, C.P. Buszello ${ }^{165}$, F. Butin ${ }^{29}$, B. Butler ${ }^{143}$, J.M. Butler ${ }^{21}$, C.M. Buttar ${ }^{53}$, J.M. Butterworth ${ }^{77}$, T. Byatt ${ }^{77}$, J. Caballero ${ }^{24}$, S. Cabrera Urbán ${ }^{166}$, M. Caccia ${ }^{89 a, 89 b, e}$, D. Caforio ${ }^{19 a, 19 b}$, O. Cakir ${ }^{3 a}$, P. Calafiura ${ }^{14}$, G. Calderini ${ }^{78}$, P. Calfayan ${ }^{98}$, R. Calkins ${ }^{106 a}$, L.P. Caloba ${ }^{23 a}$, R. Caloi ${ }^{132 a, 132 b}$, D. Calvet $^{33}$, S. Calvet ${ }^{81}$, A. Camard ${ }^{78}$, P. Camarri133a,133b, M. Cambiaghi119a,119b, D. Cameron ${ }^{117}$, J. Cammin ${ }^{20}$, S. Campana ${ }^{29}$, M. Campanelli 77 , V. Canale ${ }^{102 a, 102 b}$, F. Canelli $^{30}$, A. Canepa ${ }^{158 a}$, J. Cantero ${ }^{80}$, L. Capasso ${ }^{102 a, 102 b}$, M.D.M. Capeans Garrido ${ }^{29}$, I. Caprini ${ }^{25 a}$, M. Caprini ${ }^{25 a}$, M. Caprio ${ }^{102 a, 102 b}$, M. Capua ${ }^{36 a, 36 b}$, R. Caputo ${ }^{147}$, C. Caramarcu ${ }^{25 a}$, R. Cardarelli ${ }^{133 a}$, L. Cardiel $\operatorname{Sas}^{29}$, T. Carli ${ }^{29}$, G. Carlino ${ }^{102 a}$, L. Carminati ${ }^{89 a, 89 b}$, B. Caron $^{2, c}$, S. Caron ${ }^{48}$, C. Carpentieri ${ }^{48}$, G.D. Carrillo Montoya ${ }^{171}$, S. Carron Montero ${ }^{157}$, A.A. Carter ${ }^{75}$, J.R. Carter $^{27}$, J. Carvalho ${ }^{124 a}$, D. Casadei ${ }^{108}$, 
M.P. Casado ${ }^{11}$, M. Cascella ${ }^{122 a, 122 b}$, C. Caso ${ }^{50 a, 50 b, *}$, A.M. Castaneda Hernandez ${ }^{171}$, E. Castaneda-Miranda ${ }^{171}$, V. Castillo Gimenez ${ }^{166}$, N.F. Castro ${ }^{124 b}$, G. Cataldi ${ }^{72 a}$, F. Cataneo ${ }^{29}$, A. Catinaccio ${ }^{29}$, J.R. Catmore ${ }^{71}$, A. Cattai ${ }^{29}$, G. Cattani133a,133b, S. Caughron ${ }^{34}$, D. Cauz ${ }^{163 a, 163 c}$, A. Cavallari132a,132b ${ }^{13}$ P. Cavalleri ${ }^{78}$, D. Cavalli ${ }^{89 a}$, M. Cavalli-Sforza ${ }^{11}$, V. Cavasinni122a,122b, A. Cazzato ${ }^{72 a, 72 b}$, F. Ceradini ${ }^{134 a, 134 b}$, C. Cerna $^{83}$, A.S. Cerqueira ${ }^{23 a}$, A. Cerri ${ }^{29}$, L. Cerrito ${ }^{75}$, F. Cerutti ${ }^{47}$, M. Cervetto ${ }^{50 a, 50 b}$, S.A. Cetin ${ }^{18 b}$, F. Cevenini ${ }^{102 a, 102 b}$, A. Chafaq135a ${ }^{13}$ D. Chakraborty ${ }^{106 a}$, K. Chan ${ }^{2}$, J.D. Chapman ${ }^{27}$, J.W. Chapman ${ }^{87}$, E. Chareyre ${ }^{78}$, D.G. Charlton ${ }^{17}$, S. Charron ${ }^{93}$, S. Chatterjii ${ }^{20}$, V. Chavda ${ }^{82}$, S. Cheatham ${ }^{71}$, S. Chekanov ${ }^{5}$, S.V. Chekulaev ${ }^{158 a}$, G.A. Chelkov ${ }^{65}$, H. Chen ${ }^{24}$, L. Chen ${ }^{2}$, S. Chen ${ }^{32 c}$, T. Chen ${ }^{32 c}$, X. Chen ${ }^{171}$, S. Cheng ${ }^{32 a}$, A. Cheplakov $^{65}$, V.F. Chepurnov ${ }^{65}$, R. Cherkaoui El Moursli135d, V. Tcherniatine ${ }^{24}$, D. Chesneanu ${ }^{25 a}$, E. Cheu ${ }^{6}$, S.L. Cheung ${ }^{157}$, L. Chevalier ${ }^{136}$, F. Chevallier ${ }^{136}$, V. Chiarella ${ }^{47}$, G. Chiefari102a,102b ${ }^{10}$ L. Chikovani ${ }^{51}$, J.T. Childers ${ }^{58 a}$, A. Chilingarov ${ }^{71}$, G. Chiodini ${ }^{72 a}$, V. Chizhov ${ }^{65}$, G. Choudalakis ${ }^{30}$, S. Chouridou ${ }^{137}$, I.A. Christidi ${ }^{77}$, A. Christov $^{48}$, D. Chromek-Burckhart ${ }^{29}$, M.L. Chu ${ }^{150}$, J. Chudoba ${ }^{125}$, G. Ciapetti1 ${ }^{132 a, 132 b}$, E. Cicalini ${ }^{122 a, 122 b}$, A.K. Ciftci ${ }^{3 a}$, R. Ciftci ${ }^{3 a}$, D. Cinca ${ }^{33}$, V. Cindro ${ }^{74}$, M.D. Ciobotaru ${ }^{162}$, C. Ciocca ${ }^{19 a, 19 b}$, A. Ciocio $^{14}$, M. Cirilli ${ }^{87}$, M. Citterio ${ }^{89 a}$, A. Clark ${ }^{49}$, P.J. Clark ${ }^{45}$, W. Cleland ${ }^{123}$, J.C. Clemens ${ }^{83}$, B. Clement ${ }^{55}$, C. Clement ${ }^{145 a, 145 b}$, D. Clements ${ }^{53}$, R.W. Clifft ${ }^{129}$, Y. Coadou ${ }^{83}$, M. Cobal ${ }^{163 a, 163 c}$, A. Coccaro ${ }^{50 a, 50 b}$, J. Cochran ${ }^{64}$, P. Coe ${ }^{118}$, S. Coelli ${ }^{89 a}$, J. Coggeshall ${ }^{164}$, E. Cogneras ${ }^{179}$, C.D. Cojocaru ${ }^{28}$, J. Colas ${ }^{4}$, B. Cole ${ }^{34}$, A.P. Colijn ${ }^{105}$, C. Collard ${ }^{115}$, N.J. Collins ${ }^{17}$, C. Collins-Tooth ${ }^{53}$, J. Collot ${ }^{55}$, G. Colon ${ }^{84}$, R. Coluccia ${ }^{72 a, 72 b}$, G. Comune ${ }^{88}$, P. Conde Muiño $^{124 a}$, E. Coniavitis ${ }^{165}$, M.C. Conidi ${ }^{11}$, M. Consonni ${ }^{104}$, S. Constantinescu ${ }^{25 a}$, C. Conta ${ }^{119 a, 119 b}$, F. Conventi ${ }^{102 a, f}$, J. Cook ${ }^{29}$, M. Cooke ${ }^{34}$, B.D. Cooper ${ }^{75}$, A.M. Cooper-Sarkar ${ }^{118}$, N.J. Cooper-Smith ${ }^{76}$, K. Copic ${ }^{34}$, T. Cornelissen ${ }^{50 a, 50 b}$, M. Corradi ${ }^{19 a}$, S. Correard ${ }^{83}$, F. Corriveau ${ }^{85, g}$, A. Corso-Radu ${ }^{162}$, A. Cortes-Gonzalez ${ }^{164}$,

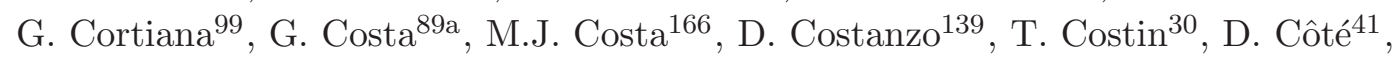
R. Coura Torres ${ }^{23 a}$, L. Courneyea ${ }^{168}$, C. Couyoumtzelis ${ }^{49}$, G. Cowan ${ }^{76}$, C. Cowden ${ }^{27}$, B.E. $\mathrm{Cox}^{82}$, K. Cranmer ${ }^{108}$, J. Cranshaw ${ }^{5}$, M. Cristinziani ${ }^{20}$, G. Crosetti $^{36 a}, 36 \mathrm{~b}$, R. Crupi ${ }^{72 a, 72 b}$, S. Crépé-Renaudin ${ }^{55}$, C. Cuenca Almenar ${ }^{174}$,

T. Cuhadar Donszelmann ${ }^{139}$, S. Cuneo ${ }^{50 a, 50 b}$, M. Curatolo ${ }^{47}$, C.J. Curtis ${ }^{17}$, P. Cwetanski ${ }^{61}$, Z. Czyczula ${ }^{174}$, S. D'Auria ${ }^{53}$, M. D'Onofrio ${ }^{73}$, A. D'Orazio ${ }^{99}$,

A. Da Rocha Gesualdi Mello ${ }^{23 a}$, P.V.M. Da Silva ${ }^{23 a}, \mathrm{C}$ Da Via ${ }^{82}$, W. Dabrowski ${ }^{37}$,

A. Dahlhoff ${ }^{48}$, T. Dai ${ }^{87}$, C. Dallapiccola ${ }^{84}$, S.J. Dallison ${ }^{129, *}$, J. Dalmau ${ }^{75}$, C.H. Daly ${ }^{138}$, M. $\operatorname{Dam}^{35}$, M. Dameri ${ }^{50 a, 50 b}$, H.O. Danielsson ${ }^{29}$, R. Dankers ${ }^{105}$, D. Dannheim ${ }^{99}$, V. Dao ${ }^{49}$, G. Darbo ${ }^{50 a}$, G.L. Darlea ${ }^{25 b}$, C. Daum ${ }^{105}$, J.P. Dauvergne ${ }^{29}$, W. Davey ${ }^{86}$,

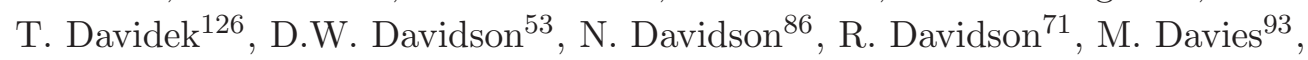
A.R. Davison ${ }^{77}$, I. Dawson ${ }^{139}$, J.W. Dawson ${ }^{5, *}$, R.K. Daya ${ }^{39}$, K. De $^{7}$, R. de Asmundis ${ }^{102 a}$, S. De Castro ${ }^{19 a, 19 b}$, P.E. De Castro Faria Salgado ${ }^{24}$, S. De Cecco ${ }^{78}$, J. de Graat ${ }^{98}$, N. De Groot ${ }^{104}$, P. de Jong ${ }^{105}$, E. De La Cruz-Burelo ${ }^{87}$, C. De La Taille ${ }^{115}$, B. De Lotto ${ }^{163 a, 163 c}$, L. De Mora ${ }^{71}$, M. De Oliveira Branco ${ }^{29}$, D. De Pedis ${ }^{132 a}$, P. de Saintignon ${ }^{55}$, A. De Salvo ${ }^{132 a}$, U. De Sanctis ${ }^{163 a, 163 c}$, A. De Santo ${ }^{148}$, J.B. De Vivie De Regie ${ }^{115}$, G. De Zorzi ${ }^{132 a, 132 b}$, S. Dean ${ }^{77}$, G. Dedes ${ }^{99}$, D.V. Dedovich ${ }^{65}$, 
P.O. Defay ${ }^{33}$, J. Degenhardt ${ }^{120}$, M. Dehchar ${ }^{118}$, M. Deile ${ }^{98}$, C. Del Papa ${ }^{163 a, 163 c}$, J. Del Peso ${ }^{80}$, T. Del Prete ${ }^{122 a, 122 b}$, A. Dell'Acqua ${ }^{29}$, L. Dell'Asta ${ }^{89 a, 89 b}$, M. Della Pietra ${ }^{102 a, f}$, D. della Volpe ${ }^{102 a, 102 b}$, M. Delmastro ${ }^{29}$, P. Delpierre ${ }^{83}$, N. Delruelle ${ }^{29}$, P.A. Delsart ${ }^{55}$, C. Deluca ${ }^{147}$, S. Demers ${ }^{174}$, M. Demichev ${ }^{65}$, B. Demirkoz ${ }^{11}$, J. Deng ${ }^{162}$, W. Deng ${ }^{24}$, S.P. Denisov ${ }^{128}$, C. Dennis ${ }^{118}$, J.E. Derkaoui ${ }^{135 c}$,

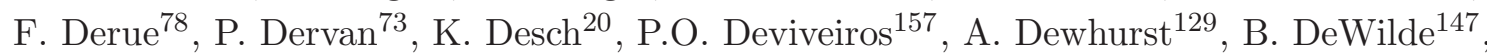
S. Dhaliwal ${ }^{157}$, R. Dhullipudi ${ }^{24, h}$, A. Di Ciaccio ${ }^{133 a, 133 b}$, L. Di Ciaccio ${ }^{4}$,

A. Di Domenico ${ }^{132 a, 132 b}$, A. Di Girolamo ${ }^{29}$, B. Di Girolamo ${ }^{29}$, S. Di Luise ${ }^{134 a, 134 b}$, A. Di Mattia ${ }^{88}$, R. Di Nardo133a,133b, A. Di Simone ${ }^{133 a, 133 b}$, R. Di Sipio ${ }^{19 a, 19 b}$, M.A. Diaz ${ }^{31 a}$, M.M. Diaz Gomez ${ }^{49}$, F. Diblen ${ }^{18 c}$, E.B. Diehl ${ }^{87}$, H. Dietl ${ }^{99}$, J. Dietrich ${ }^{48}$, T.A. Dietzsch ${ }^{58 a}$, S. Diglio ${ }^{115}$, K. Dindar Yagci $^{39}$, J. Dingfelder ${ }^{48}$, C. Dionisi ${ }^{132 a, 132 b}$,

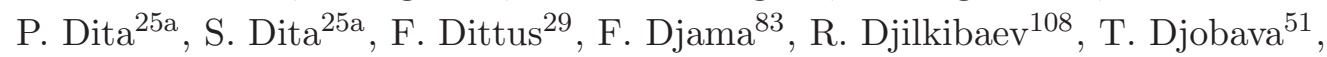
M.A.B. do Vale ${ }^{23 a}$, A. Do Valle Wemans ${ }^{124 a}$, T.K.O. Doan ${ }^{4}$, M. Dobbs ${ }^{85}$, R. Dobinson ${ }^{29, *}$, D. Dobos ${ }^{29}$, E. Dobson ${ }^{29}$, M. Dobson ${ }^{162}$, J. Dodd ${ }^{34}$, O.B. Dogan ${ }^{18 a}, *$, C. Doglioni ${ }^{118}$, T. Doherty ${ }^{53}$, Y. Doi ${ }^{66}$, J. Dolejsi ${ }^{126}$, I. Dolenc ${ }^{74}$, Z. Dolezal ${ }^{126}$, B.A. Dolgoshein ${ }^{96}$, T. Dohmae ${ }^{154}$, E. Domingo ${ }^{11}$, M. Donega ${ }^{120}$, J. Donini ${ }^{55}$, J. Dopke ${ }^{173}$, A. Doria ${ }^{102 a}$, A. Dos Anjos ${ }^{171}$, M. Dosil ${ }^{11}$, A. Dotti ${ }^{122 a, 122 b}$, M.T. Dova ${ }^{70}$, J.D. Dowell ${ }^{17}$, A. Doxiadis ${ }^{105}$, A.T. Doyle ${ }^{53}$, Z. Drasal ${ }^{126}$, J. Drees ${ }^{173}$, N. Dressnandt ${ }^{120}$, H. Drevermann ${ }^{29}$, C. Driouichi ${ }^{35}$, M. Dris ${ }^{9}$, J.G. Drohan ${ }^{77}$, J. Dubbert ${ }^{99}$, T. Dubbs ${ }^{137}$, S. Dube ${ }^{14}$, E. Duchovni ${ }^{170}$, G. Duckeck ${ }^{98}$, A. Dudarev ${ }^{29}$, F. Dudziak ${ }^{115}$, M. Dührssen ${ }^{29}$, H. Dür ${ }^{62}$, I.P. Duerdoth ${ }^{82}$, L. Duflot ${ }^{115}$, M-A. Dufour ${ }^{85}$, M. Dunford ${ }^{30}$, H. Duran Yildiz ${ }^{3 b}$, A. Dushkin ${ }^{22}$, R. Duxfield ${ }^{139}$, M. Dwuznik ${ }^{37}$, F. Dydak ${ }^{29}$, D. Dzahini ${ }^{55}$, M. Düren ${ }^{52}$, W.L. Ebenstein ${ }^{44}$, J. Ebke ${ }^{98}$, S. Eckert ${ }^{48}$, S. Eckweiler ${ }^{81}$, K. Edmonds ${ }^{81}$, C.A. Edwards ${ }^{76}$,

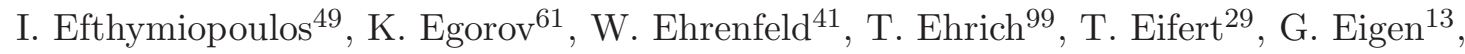
K. Einsweiler ${ }^{14}$, E. Eisenhandler ${ }^{75}$, T. Ekelof ${ }^{165}$, M. El Kacimi ${ }^{4}$, M. Ellert ${ }^{165}$, S. Elles ${ }^{4}$, F. Ellinghaus ${ }^{81}$, K. Ellis ${ }^{75}$, N. Ellis ${ }^{29}$, J. Elmsheuser ${ }^{98}$, M. Elsing ${ }^{29}$, R. Ely ${ }^{14}$, D. Emeliyanov ${ }^{129}$, R. Engelmann ${ }^{147}$, A. Engl ${ }^{98}$, B. $\operatorname{Epp}^{62}$, A. Eppig ${ }^{87}$, J. Erdmann ${ }^{54}$, A. Ereditato ${ }^{16}$, V. Eremin ${ }^{97}$, D. Eriksson ${ }^{145 a}$, I. Ermoline ${ }^{88}$, J. Ernst ${ }^{1}$, M. Ernst ${ }^{24}$, J. Ernwein ${ }^{136}$, D. Errede ${ }^{164}$, S. Errede ${ }^{164}$, E. Ertel ${ }^{81}$, M. Escalier ${ }^{115}$, C. Escobar ${ }^{166}$, X. Espinal Curull ${ }^{11}$, B. Esposito ${ }^{47}$, F. Etienne ${ }^{83}$, A.I. Etienvre ${ }^{136}$, E. Etzion ${ }^{152}$, H. Evans ${ }^{61}$, V.N. Evdokimov ${ }^{128}$, L. Fabbri ${ }^{19 a, 19 b}$, C. Fabre ${ }^{29}$, K. Facius ${ }^{35}$, R.M. Fakhrutdinov ${ }^{128}$, S. Falciano ${ }^{132 a}$, A.C. Falou ${ }^{115}$, Y. Fang ${ }^{171}$, M. Fanti ${ }^{89 a}$,89b, A. Farbin ${ }^{7}$, A. Farilla ${ }^{134 a}$, J. Farley ${ }^{147}$, T. Farooque ${ }^{157}$, S.M. Farrington ${ }^{118}$, P. Farthouat ${ }^{29}$, P. Fassnacht ${ }^{29}$, D. Fassouliotis ${ }^{8}$, B. Fatholahzadeh ${ }^{157}$, L. Fayard ${ }^{115}$, F. Fayette $^{54}$, R. Febbraro ${ }^{33}$, P. Federic ${ }^{144 a}$, O.L. Fedin ${ }^{121}$, I. Fedorko ${ }^{29}$, W. Fedorko ${ }^{29}$, L. Feligioni ${ }^{83}$, C.U. Felzmann ${ }^{86}$, C. Feng ${ }^{32 d}$, E.J. Feng ${ }^{30}$, A.B. Fenyuk ${ }^{128}$, J. Ferencei ${ }^{144 b}$, J. Ferland ${ }^{93}$, B. Fernandes ${ }^{124 a}$, W. Fernando ${ }^{109}$, S. Ferrag ${ }^{53}$, J. Ferrando ${ }^{118}$, V. Ferrara ${ }^{41}$, A. Ferrari ${ }^{165}$, P. Ferrari ${ }^{105}$, R. Ferrari ${ }^{119 a}$, A. Ferrer ${ }^{166}$, M.L. Ferrer ${ }^{47}$, D. Ferrere ${ }^{49}$, C. Ferretti ${ }^{87}$, F. Ferro ${ }^{50 a, 50 b}$, M. Fiascaris ${ }^{118}$, F. Fiedler ${ }^{81}$, A. Filipčič ${ }^{74}$, A. Filippas ${ }^{9}$, F. Filthaut ${ }^{104}$, M. Fincke-Keeler ${ }^{168}$, M.C.N. Fiolhais ${ }^{124 a}$, L. Fiorini ${ }^{11}$, A. Firan ${ }^{39}$, G. Fischer ${ }^{41}$, P. Fischer ${ }^{20}$, M.J. Fisher ${ }^{109}$, S.M. Fisher ${ }^{129}$, J. Flammer ${ }^{29}$, M. Flechl ${ }^{48}$, I. Fleck ${ }^{141}$, J. Fleckner ${ }^{81}$, P. Fleischmann ${ }^{172}$, S. Fleischmann ${ }^{20}$, F. Fleuret ${ }^{78}$, T. Flick ${ }^{173}$, L.R. Flores Castillo ${ }^{171}$, M.J. Flowerdew ${ }^{99}$, F. Föhlisch ${ }^{58 a}$, M. Fokitis ${ }^{9}$, 
T. Fonseca Martin ${ }^{76}$, J. Fopma ${ }^{118}$, D.A. Forbush ${ }^{138}$, A. Formica ${ }^{136}$, A. Forti ${ }^{82}$, D. Fortin ${ }^{158 a}$, J.M. Foster ${ }^{82}$, D. Fournier ${ }^{115}$, A. Foussat ${ }^{29}$, A.J. Fowler ${ }^{44}$, K. Fowler ${ }^{137}$, H. Fox ${ }^{71}$, P. Francavilla122a,122b, S. Franchino ${ }^{119 a, 119 b}$, D. Francis ${ }^{29}$, M. Franklin ${ }^{57}$, S. Franz ${ }^{29}$, M. Fraternali ${ }^{119 a, 119 b}$, S. Fratina ${ }^{120}$, J. Freestone ${ }^{82}$, S.T. French ${ }^{27}$, R. Froeschl ${ }^{29}$, D. Froidevaux ${ }^{29}$, J.A. Frost ${ }^{27}$, C. Fukunaga ${ }^{155}$, E. Fullana Torregrosa ${ }^{5}$, J. Fuster ${ }^{166}$, C. Gabaldon ${ }^{80}$, O. Gabizon ${ }^{170}$, T. Gadfort ${ }^{24}$, S. Gadomski ${ }^{49}$, G. Gagliardi ${ }^{50 a, 50 b}$, P. Gagnon ${ }^{61}$, C. Galea ${ }^{98}$, E.J. Gallas ${ }^{118}$, M.V. Gallas ${ }^{29}$, V. Gallo ${ }^{16}$, B.J. Gallop ${ }^{129}$, P. Gallus ${ }^{125}$, E. Galyaev ${ }^{40}$, K.K. Gan ${ }^{109}$, Y.S. Gao ${ }^{143, i}$, V.A. Gapienko ${ }^{128}$, A. Gaponenko ${ }^{14}$, M. Garcia-Sciveres ${ }^{14}$, C. García ${ }^{166}$, J.E. García Navarro ${ }^{49}$, V. Garde ${ }^{33}$, R.W. Gardner ${ }^{30}$, N. Garelli ${ }^{29}$, H. Garitaonandia ${ }^{105}$, V. Garonne ${ }^{29}$, J. Garvey ${ }^{17}$, C. Gatti ${ }^{47}$, G. Gaudio ${ }^{119 a}$, O. Gaumer ${ }^{49}$, V. Gautard ${ }^{136}$, P. Gauzzi1 ${ }^{132 a, 132 b}$, I.L. Gavrilenko ${ }^{94}$, C. Gay ${ }^{167}$, G. Gaycken ${ }^{20}$, J-C. Gayde ${ }^{29}$, E.N. Gazis ${ }^{9}$, P. Ge ${ }^{32 d}$, C.N.P. Gee ${ }^{129}$, Ch. Geich-Gimbel ${ }^{20}$, K. Gellerstedt ${ }^{145 a, 145 b}$, C. Gemme ${ }^{50 a}$, M.H. Genest ${ }^{98}$, S. Gentile ${ }^{132 a, 132 b}$, F. Georgatos ${ }^{9}$, S. George ${ }^{76}$, P. Gerlach ${ }^{173}$, A. Gershon ${ }^{152}$, C. Geweniger ${ }^{58 a}$, H. Ghazlane ${ }^{135 d}$, P. Ghez ${ }^{4}$, N. Ghodbane ${ }^{33}$, B. Giacobbe ${ }^{19 a}$, S. Giagu ${ }^{132 a, 132 b}$, V. Giakoumopoulou ${ }^{8}$, V. Giangiobbe ${ }^{122 a, 122 b}$, F. Gianotti ${ }^{29}$, B. Gibbard ${ }^{24}$, A. Gibson ${ }^{157}$, S.M. Gibson ${ }^{118}$, G.F. Gieraltowski ${ }^{5}$, L.M. Gilbert ${ }^{118}$, M. Gilchriese ${ }^{14}$, O. Gildemeister ${ }^{29}$, V. Gilewsky ${ }^{91}$, A.R. Gillman ${ }^{129}$, D.M. Gingrich ${ }^{2, c}$, J. Ginzburg ${ }^{152}$, N. Giokaris ${ }^{8}$, M.P. Giordani ${ }^{163 a, 163 c}$, R. Giordano102a,102b , F.M. Giorgi ${ }^{15}$, P. Giovannini ${ }^{99}$, P.F. Giraud ${ }^{29}$, P. Girtler ${ }^{62}$, D. Giugni ${ }^{89 a}$, P. Giusti ${ }^{19 a}$, B.K. Gjelsten ${ }^{117}$, L.K. Gladilin ${ }^{97}$, C. Glasman ${ }^{80}$, A. Glazov ${ }^{41}$, K.W. Glitza ${ }^{173}$, G.L. Glonti ${ }^{65}$, K.G. Gnanvo ${ }^{75}$, J. Godfrey ${ }^{142}$, J. Godlewski ${ }^{29}$, M. Goebel ${ }^{41}$, T. Göpfert ${ }^{43}$, C. Goeringer ${ }^{81}$, C. Gössling ${ }^{42}$, T. Göttfert ${ }^{99}$, V. Goggi ${ }^{119 a, 119 b, j}$, S. Goldfarb ${ }^{87}$, D. Goldin ${ }^{39}$, T. Golling ${ }^{174}$, N.P. Gollub ${ }^{29}$, S.N. Golovnia ${ }^{128}$, A. Gomes ${ }^{124 a}$, L.S. Gomez Fajardo ${ }^{41}$, R. Gonçalo ${ }^{76}$, L. Gonella ${ }^{20}$, C. Gong ${ }^{32 b}$, A. Gonidec ${ }^{29}$, S. González de la $\mathrm{Hoz}^{166}$, M.L. Gonzalez Silva ${ }^{26}$, B. Gonzalez-Pineiro ${ }^{88}$, S. Gonzalez-Sevilla ${ }^{49}$, J.J. Goodson ${ }^{147}$, L. Goossens ${ }^{29}$, P.A. Gorbounov ${ }^{157}$, H.A. Gordon ${ }^{24}$, I. Gorelov ${ }^{103}$, G. Gorfine ${ }^{173}$, B. Gorini ${ }^{29}$, E. Gorini ${ }^{72 a, 72 b}$, A. Gorišek ${ }^{74}$, E. Gornicki ${ }^{38}$, S.A. Gorokhov ${ }^{128}$, B.T. Gorski ${ }^{29}$, V.N. Goryachev ${ }^{128}$, B. Gosdzik ${ }^{41}$, M. Gosselink ${ }^{105}$, M.I. Gostkin ${ }^{65}$, M. Gouanère ${ }^{4}$, I. Gough Eschrich ${ }^{162}$, M. Gouighri ${ }^{135 a}$, D. Goujdami ${ }^{135 a}$, M.P. Goulette ${ }^{49}$, A.G. Goussiou ${ }^{138}$, C. Goy ${ }^{4}$, I. Grabowska-Bold ${ }^{162, d}$, V. Grabski ${ }^{175}$, P. Grafström ${ }^{29}$, C. Grah ${ }^{173}$, K-J. Grahn ${ }^{146}$, F. Grancagnolo ${ }^{72 a}$, S. Grancagnolo ${ }^{15}$, V. Grassi ${ }^{147}$, V. Gratchev ${ }^{121}$, N. Grau ${ }^{34}$, H.M. Gray ${ }^{34, k}$, J.A. Gray ${ }^{147}$, E. Graziani134a, B. Green ${ }^{76}$, D. Greenfield ${ }^{129}$, T. Greenshaw ${ }^{73}$, Z.D. Greenwood ${ }^{24, h}$, I.M. Gregor ${ }^{41}$, P. Grenier ${ }^{143}$, A. Grewal ${ }^{118}$, E. Griesmayer ${ }^{46}$, J. Griffiths ${ }^{138}$, N. Grigalashvilii ${ }^{65}$, A.A. Grillo ${ }^{137}$, K. Grimm ${ }^{147}$, S. Grinstein ${ }^{11}$, P.L.Y. Gris ${ }^{33}$, Y.V. Grishkevich ${ }^{97}$, L.S. Groer ${ }^{157}$, J. Grognuz ${ }^{29}$, M. Groh ${ }^{99}$, M. Groll ${ }^{81}$, E. Gross ${ }^{170}$, J. Grosse-Knetter ${ }^{54}$, J. Groth-Jensen ${ }^{79}$, M. Gruwe ${ }^{29}$, K. Grybel ${ }^{141}$, V.J. Guarino ${ }^{5}$, C. Guicheney ${ }^{33}$,

A. Guida ${ }^{72 a, 72 b}$, T. Guillemin ${ }^{4}$, H. Guler ${ }^{85, l}$, J. Gunther ${ }^{125}$, B. Guo ${ }^{157}$, A. Gupta ${ }^{30}$, Y. Gusakov ${ }^{65}$, V.N. Gushchin ${ }^{128}$, A. Gutierrez ${ }^{93}$, P. Gutierrez ${ }^{111}$, N. Guttman ${ }^{152}$,

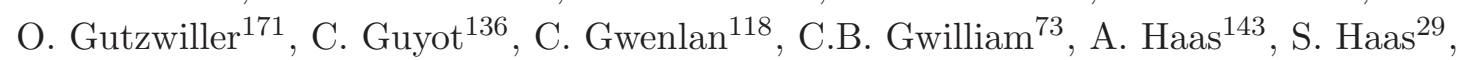
C. Haber ${ }^{14}$, G. Haboubi ${ }^{123}$, R. Hackenburg ${ }^{24}$, H.K. Hadavand ${ }^{39}$, D.R. Hadley ${ }^{17}$, C. Haeberli ${ }^{16}$, P. Haefner ${ }^{99}$, R. Härtel ${ }^{99}$, F. Hahn ${ }^{29}$, S. Haider ${ }^{29}$, Z. Hajduk ${ }^{38}$, 
H. Hakobyan ${ }^{175}$, R.H. Hakobyan ${ }^{2}$, J. Haller ${ }^{41, m}$, G.D. Hallewell ${ }^{83}$, K. Hamacher ${ }^{173}$,

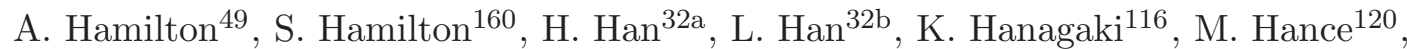
C. Handel ${ }^{81}$, P. Hanke ${ }^{58 a}$, C.J. Hansen ${ }^{165}$, J.R. Hansen ${ }^{35}$, J.B. Hansen ${ }^{35}$, J.D. Hansen ${ }^{35}$, P.H. Hansen ${ }^{35}$, T. Hansl-Kozanecka ${ }^{137}$, P. Hansson ${ }^{143}$, K. Hara ${ }^{159}$, G.A. Hare ${ }^{137}$, T. Harenberg ${ }^{173}$, R. Harper ${ }^{139}$, R.D. Harrington ${ }^{21}$, O.M. Harris ${ }^{138}$, K Harrison ${ }^{17}$, J.C. Hart ${ }^{129}$, J. Hartert ${ }^{48}$, F. Hartjes ${ }^{105}$, T. Haruyama ${ }^{66}$, A. Harvey ${ }^{56}$, S. Hasegawa ${ }^{101}$,

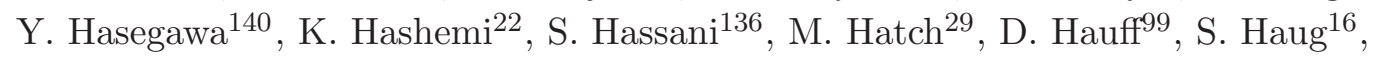
M. Hauschild ${ }^{29}$, R. Hauser ${ }^{88}$, M. Havranek ${ }^{125}$, B.M. Hawes ${ }^{118}$, C.M. Hawkes ${ }^{17}$, R.J. Hawkings ${ }^{29}$, D. Hawkins ${ }^{162}$, T. Hayakawa ${ }^{67}$, H.S. Hayward ${ }^{73}$, S.J. Haywood ${ }^{129}$,

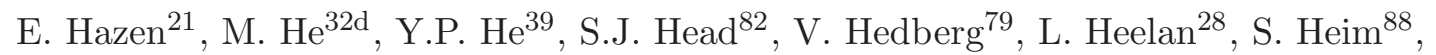
B. Heinemann ${ }^{14}$, F.E.W. Heinemann ${ }^{118}$, S. Heisterkamp ${ }^{35}$, L. Helary ${ }^{4}$, M. Heldmann ${ }^{48}$, M. Heller ${ }^{115}$, S. Hellman ${ }^{145 a, 145 b}$, C. Helsens ${ }^{11}$, T. Hemperek ${ }^{20}$, R.C.W. Henderson ${ }^{71}$, P.J. Hendriks ${ }^{105}$, M. Henke ${ }^{58 a}$, A. Henrichs ${ }^{54}$, A.M. Henriques Correia ${ }^{29}$,

S. Henrot-Versille ${ }^{115}$, F. Henry-Couannier ${ }^{83}$, C. Hensel ${ }^{54}$, T. Henß ${ }^{173}$, Y. Hernández Jiménez $^{166}$, A.D. Hershenhorn ${ }^{151}$, G. Herten ${ }^{48}$, R. Hertenberger ${ }^{98}$, L. Hervas ${ }^{29}$, M. Hess ${ }^{16}$, N.P. Hessey ${ }^{105}$, A. Hidvegi ${ }^{145 a}$, E. Higón-Rodriguez ${ }^{166}$, D. Hill ${ }^{5, *}$, J.C. Hill ${ }^{27}$, N. Hill ${ }^{5}$, K.H. Hiller ${ }^{41}$, S. Hillert ${ }^{145 a, 145 b}$, S.J. Hillier ${ }^{17}$, I. Hinchliffe ${ }^{14}$, D. Hindson ${ }^{118}$,

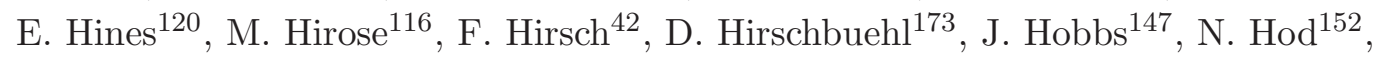
M.C. Hodgkinson ${ }^{139}$, P. Hodgson ${ }^{139}$, A. Hoecker ${ }^{29}$, M.R. Hoeferkamp ${ }^{103}$, J. Hoffman ${ }^{39}$, D. Hoffmann ${ }^{83}$, M. Hohlfeld ${ }^{81}$, M. Holder ${ }^{141}$, T.I. Hollins ${ }^{17}$, G. Hollyman ${ }^{76}$,

A. Holmes ${ }^{118}$, S.O. Holmgren ${ }^{145 a}$, T. Holy ${ }^{127}$, J.L. Holzbauer ${ }^{88}$, R.J. Homer ${ }^{17}$, Y. Homma ${ }^{67}$, T. Horazdovsky ${ }^{127}$, T. Hori ${ }^{67}$, C. Horn ${ }^{143}$, S. Horner ${ }^{48}$, S. Horvat ${ }^{99}$,

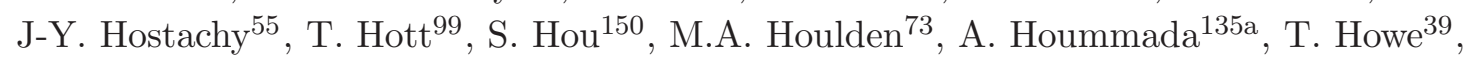
D.F. Howell ${ }^{118}$, J. Hrivnac ${ }^{115}$, I. Hruska ${ }^{125}$, T. Hryn'ova ${ }^{4}$, P.J. Hsu ${ }^{174}$, S.-C. Hsu ${ }^{14}$, G.S. Huang ${ }^{111}$, Z. Hubacek ${ }^{127}$, F. Hubaut ${ }^{83}$, F. Huegging ${ }^{20}$, T.B. Huffman ${ }^{118}$, E.W. Hughes ${ }^{34}$, G. Hughes ${ }^{71}$, R.E. Hughes-Jones ${ }^{82}$, M. Huhtinen ${ }^{29}$, P. Hurst ${ }^{57}$, M. Hurwitz ${ }^{30}$, U. Husemann ${ }^{41}$, N. Huseynov ${ }^{10}$, J. Huston ${ }^{88}$, J. Huth ${ }^{57}$, G. Iacobucci ${ }^{102 a}$, G. Iakovidis ${ }^{9}$, M. Ibbotson ${ }^{82}$, I. Ibragimov ${ }^{141}$, R. Ichimiya ${ }^{67}$, L. Iconomidou-Fayard ${ }^{115}$, J. Idarraga ${ }^{158 b}$, M. Idzik ${ }^{37}$, P. Iengo ${ }^{4}$, O. Igonkina ${ }^{105}$, Y. Ikegami ${ }^{66}$, M. Ikeno $^{66}$, Y. Ilchenko ${ }^{39}$, D. Iliadis ${ }^{153}$, D. Imbault ${ }^{78}$, M. Imhaeuser ${ }^{173}$, M. Imori ${ }^{154}$, T. Ince ${ }^{20}$, J. Inigo-Golfin ${ }^{29}$, P. Ioannou ${ }^{8}$, M. Iodice ${ }^{134 a}$, G. Ionescu ${ }^{4}$, A. Irles Quiles ${ }^{166}$, K. Ishii ${ }^{66}$, A. Ishikawa ${ }^{67}$, M. Ishino ${ }^{66}$, Y. Ishizawa ${ }^{158 a}$, R. Ishmukhametov ${ }^{39}$, T. Isobe ${ }^{154}$,

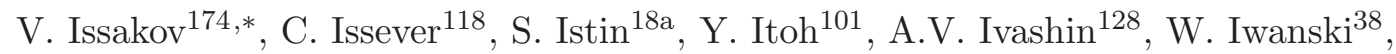
H. Iwasaki ${ }^{66}$, J.M. Izen ${ }^{40}$, V. Izzo ${ }^{102 a}$, B. Jackson ${ }^{120}$, J.N. Jackson ${ }^{73}$, P. Jackson ${ }^{143}$, M.R. Jaekel ${ }^{29}$, M. Jahoda ${ }^{125}$, V. Jain ${ }^{61}$, K. Jakobs ${ }^{48}$, S. Jakobsen ${ }^{35}$, J. Jakubek ${ }^{127}$, D.K. Jana ${ }^{111}$, E. Jankowski ${ }^{157}$, E. Jansen ${ }^{77}$, A. Jantsch ${ }^{99}$, M. Janus ${ }^{48}$, R.C. Jared ${ }^{171}$, G. Jarlskog ${ }^{79}$, L. Jeanty ${ }^{57}$, K. Jelen ${ }^{37}$, I. Jen-La Plante ${ }^{30}$, P. Jenni ${ }^{29}$, A. Jeremie ${ }^{4}$, P. Jez ${ }^{35}$, S. Jézéquel ${ }^{4}$, W. Ji ${ }^{79}$, J. Jia ${ }^{147}$, Y. Jiang ${ }^{32 b}$, M. Jimenez Belenguer ${ }^{29}$, G. Jin ${ }^{32 b}$, S. Jin ${ }^{32 a}$, O. Jinnouchi ${ }^{156}$, D. Joffe ${ }^{39}$, L.G. Johansen ${ }^{13}$, M. Johansen ${ }^{145 a, 145 b}$, K.E. Johansson ${ }^{145 a}$, P. Johansson ${ }^{139}$, S Johnert ${ }^{41}$, K.A. Johns ${ }^{6}$, K. Jon-And ${ }^{145 a, 145 b}$, G. Jones ${ }^{82}$, M. Jones ${ }^{118}$, R.W.L. Jones ${ }^{71}$, T.W. Jones ${ }^{77}$, T.J. Jones ${ }^{73}$, O. Jonsson ${ }^{29}$, K.K. Joo ${ }^{157, n}$, D. Joos ${ }^{48}$, C. Joram ${ }^{29}$, P.M. Jorge ${ }^{124 a}$, S. Jorgensen ${ }^{11}$, V. Juranek ${ }^{125}$, P. Jussel ${ }^{62}$, V.V. Kabachenko ${ }^{128}$, S. Kabana ${ }^{16}$, M. Kaci ${ }^{166}$, A. Kaczmarska ${ }^{38}$, M. Kado ${ }^{115}$, 
H. Kagan ${ }^{109}$, M. Kagan ${ }^{57}$, S. Kaiser ${ }^{99}$, E. Kajomovitz ${ }^{151}$, S. Kalinin ${ }^{173}$, L.V. Kalinovskaya ${ }^{65}$, A. Kalinowski ${ }^{130}$, S. Kama ${ }^{41}$, H. Kambara ${ }^{49}$, N. Kanaya ${ }^{154}$, M. Kaneda ${ }^{154}$, V.A. Kantserov ${ }^{96}$, J. Kanzaki ${ }^{66}$, B. Kaplan ${ }^{174}$, A. Kapliy ${ }^{30}$, J. Kaplon ${ }^{29}$, D. $\operatorname{Kar}^{43}$, M. Karagounis ${ }^{20}$, M. Karagoz Unel ${ }^{118}$, M. Karnevskiy ${ }^{41}$, K. Karr ${ }^{5}$, V. Kartvelishvili ${ }^{71}$, A.N. Karyukhin ${ }^{128}$, L. Kashif ${ }^{57}$, A. Kasmi ${ }^{39}$, R.D. Kass ${ }^{109}$, A. Kastanas ${ }^{13}$, M. Kastoryano ${ }^{174}$, M. Kataoka ${ }^{4}$, Y. Kataoka ${ }^{154}$, E. Katsoufis ${ }^{9}$, J. Katzy ${ }^{41}$, V. Kaushik ${ }^{6}$, K. Kawagoe ${ }^{67}$, T. Kawamoto ${ }^{154}$, G. Kawamura ${ }^{81}$, M.S. Kayl ${ }^{105}$, F. Kayumov ${ }^{94}$, V.A. Kazanin ${ }^{107}$, M.Y. Kazarinov ${ }^{65}$, S.I. Kazi ${ }^{86}$, J.R. Keates ${ }^{82}$, R. Keeler ${ }^{168}$, P.T. Keener ${ }^{120}$, R. Kehoe ${ }^{39}$, M. Keil ${ }^{54}$, G.D. Kekelidze ${ }^{65}$, M. Kelly ${ }^{82}$, J. Kennedy ${ }^{98}$, M. Kenyon ${ }^{53}$, O. Kepka ${ }^{125}$, N. Kerschen ${ }^{29}$, B.P. Kerševan ${ }^{74}$, S. Kersten ${ }^{173}$, K. Kessoku ${ }^{154}$, C. Ketterer ${ }^{48}$, M. Khakzad ${ }^{28}$, F. Khalil-zada ${ }^{10}$, H. Khandanyan ${ }^{164}$, A. Khanov ${ }^{112}$, D. Kharchenko ${ }^{65}$, A. Khodinov ${ }^{147}$, A.G. Kholodenko ${ }^{128}$, A. Khomich ${ }^{58 a}$, G. Khoriauli ${ }^{20}$, N. Khovanskiy ${ }^{65}$, V. Khovanskiy ${ }^{95}$, E. Khramov ${ }^{65}$, J. Khubua ${ }^{51}$,

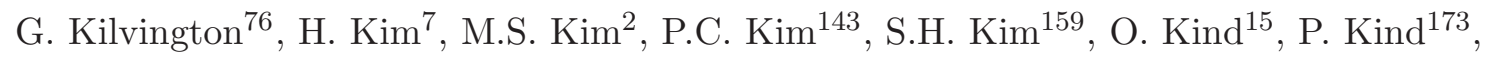
B.T. King ${ }^{73}$, M. King ${ }^{67}$, J. Kirk ${ }^{129}$, G.P. Kirsch ${ }^{118}$, L.E. Kirsch ${ }^{22}$, A.E. Kiryunin ${ }^{99}$, D. Kisielewska ${ }^{37}$, B. Kisielewski ${ }^{38}$, T. Kittelmann ${ }^{123}$, A.M. Kiver ${ }^{128}$, H. Kiyamura ${ }^{67}$, E. Kladiva ${ }^{144 b}$, J. Klaiber-Lodewigs ${ }^{42}$, M. Klein ${ }^{73}$, U. Klein ${ }^{73}$, K. Kleinknecht ${ }^{81}$, M. Klemetti ${ }^{85}$, A. Klier ${ }^{170}$, A. Klimentov ${ }^{24}$, R. Klingenberg ${ }^{42}$, E.B. Klinkby ${ }^{44}$, T. Klioutchnikova ${ }^{29}$, P.F. Klok ${ }^{104}$, S. Klous ${ }^{105}$, E.-E. Kluge ${ }^{58 a}$, T. Kluge ${ }^{73}$, P. Kluit ${ }^{105}$, S. Kluth ${ }^{99}$, N.S. Knecht ${ }^{157}$, E. Kneringer ${ }^{62}$, J. Knobloch ${ }^{29}$, B.R. Ko ${ }^{44}$, T. Kobayashi ${ }^{154}$, M. Kobel ${ }^{43}$, B. Koblitz ${ }^{29}$, M. Kocian ${ }^{143}$, A. Kocnarr ${ }^{113}$, P. Kodys ${ }^{126}$, K. Köneke ${ }^{41}$, A.C. König ${ }^{104}$, S. Koenig ${ }^{81}$, S. König ${ }^{48}$, L. Köpke ${ }^{81}$, F. Koetsveld ${ }^{104}$, P. Koevesarki ${ }^{20}$, T. Koffas ${ }^{29}$, E. Koffeman ${ }^{105}$, F. Kohn ${ }^{54}$, Z. Kohout ${ }^{127}$, T. Kohriki ${ }^{66}$, T. Koi ${ }^{143}$, T. Kokott ${ }^{20}$, G.M. Kolachev ${ }^{107, *}$, H. Kolanoski ${ }^{15}$, V. Kolesnikov ${ }^{65}$, I. Koletsou ${ }^{4}$, J. Koll ${ }^{88}$, D. Kollar ${ }^{29}$, M. Kollefrath ${ }^{48}$, S. Kolos ${ }^{162, o}$, S.D. Kolya ${ }^{82}$, A.A. Komar ${ }^{94}$, J.R. Komaragiri ${ }^{142}$, T. Kondo ${ }^{66}$, T. Kono ${ }^{41, m}$, A.I. Kononov ${ }^{48}$, R. Konoplich ${ }^{108}$, S.P. Konovalov ${ }^{94}$, N. Konstantinidis ${ }^{77}$, A. Kootz ${ }^{173}$, S. Koperny ${ }^{37}$, S.V. Kopikov ${ }^{128}$, K. Korcy ${ }^{38}$, K. Kordas ${ }^{153}$, V. Koreshev ${ }^{128}$, A. Korn ${ }^{14}$, I. Korolkov ${ }^{11}$, E.V. Korolkova ${ }^{139}$, V.A. Korotkov ${ }^{128}$, H. Korsmo ${ }^{79}$, O. Kortner ${ }^{99}$, P. Kostka ${ }^{41}$, V.V. Kostyukhin ${ }^{20}$, M.J. Kotamäki ${ }^{29}$, D. Kotchetkov ${ }^{22}$, S. Kotov ${ }^{99}$, V.M. Kotov ${ }^{65}$, K.Y. Kotov ${ }^{107}$, C. Kourkoumelis ${ }^{8}$, A. Koutsman ${ }^{105}$, R. Kowalewski ${ }^{168}$, H. Kowalski ${ }^{41}$, T.Z. Kowalski ${ }^{37}$, W. Kozanecki ${ }^{136}$, A.S. Kozhin ${ }^{128}$, V. Kral ${ }^{127}$, V.A. Kramarenko ${ }^{97}$, G. Kramberger ${ }^{74}$, O. Krasel ${ }^{42}$, M.W. Krasny ${ }^{78}$, A. Krasznahorkay ${ }^{108}$, J. Kraus ${ }^{88}$, A. Kreisel ${ }^{152}$, F. Krejci ${ }^{127}$, J. Kretzschmar ${ }^{73}$, N. Krieger ${ }^{54}$, P. Krieger ${ }^{157}$, G. Krobath ${ }^{98}$, K. Kroeninger ${ }^{54}$, H. Kroha ${ }^{99}$, J. Kroll ${ }^{120}$, J. Kroseberg ${ }^{20}$, J. Krstic ${ }^{12 a}$, U. Kruchonak ${ }^{65}$, H. Krüger ${ }^{20}$, Z.V. Krumshteyn ${ }^{65}$, A. Kruth ${ }^{20}$, T. Kubota ${ }^{154}$, S. Kuehn ${ }^{48}$, A. Kugel ${ }^{58 c}$, T. Kuhl ${ }^{173}$, D. Kuhn ${ }^{62}$, V. Kukhtin ${ }^{65}$, Y. Kulchitsky ${ }^{90}$, S. Kuleshov ${ }^{31 b}$, C. Kummer ${ }^{98}$, M. Kuna ${ }^{83}$, N. Kundu ${ }^{118}$, J. Kunkle ${ }^{120}$, A. Kupco ${ }^{125}$, H. Kurashige ${ }^{67}$, M. Kurata ${ }^{159}$, L.L. Kurchaninov ${ }^{158 a}$, Y.A. Kurochkin ${ }^{90}$, V. Kus ${ }^{125}$, W. Kuykendall ${ }^{138}$, M. Kuze ${ }^{156}$, P. Kuzhir ${ }^{91}$, E. Kuznetsova ${ }^{132 a, 132 b}$, O. Kvasnicka ${ }^{125}$, R. Kwee ${ }^{15}$, A. La Rosa ${ }^{29}$, L. La Rotonda ${ }^{36 a, 36 b}$, L. Labarga ${ }^{80}$, J. Labbe ${ }^{4}$, C. Lacasta ${ }^{166}$, F. Lacava ${ }^{132 a, 132 b}$,

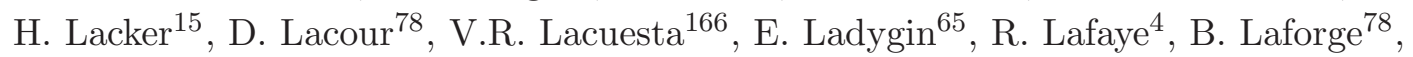
T. Lagouri ${ }^{80}$, S. Lai ${ }^{48}$, M. Lamanna ${ }^{29}$, M. Lambacher ${ }^{98}$, C.L. Lampen ${ }^{6}$, W. Lampl ${ }^{6}$, 
E. Lancon ${ }^{136}$, U. Landgraf ${ }^{48}$, M.P.J. Landon ${ }^{75}$, H. Landsman ${ }^{151}$, J.L. Lane ${ }^{82}$, A.J. Lankford ${ }^{162}$, F. Lanni ${ }^{24}$, K. Lantzsch ${ }^{29}$, A. Lanza ${ }^{119 a}$, V.V. Lapin ${ }^{128, *}$, S. Laplace ${ }^{4}$, C. Lapoire $^{83}$, J.F. Laporte ${ }^{136}$, T. Lari ${ }^{89 a}$, A.V. Larionov ${ }^{128}$, A. Larner ${ }^{118}$, C. Lasseur ${ }^{29}$, M. Lassnig ${ }^{29}$, W. Lau ${ }^{118}$, P. Laurelli ${ }^{47}$, A. Lavorato ${ }^{118}$, W. Lavrijsen ${ }^{14}$, P. Laycock ${ }^{73}$, A.B. Lazarev ${ }^{65}$, A. Lazzaro ${ }^{89 a, 89 b}$, O. Le Dortz ${ }^{78}$, E. Le Guirriec ${ }^{83}$, C. Le Maner ${ }^{157}$, E. Le Menedeu ${ }^{136}$, M. Le Vine ${ }^{24}$, M. Leahu ${ }^{29}$, A. Lebedev ${ }^{64}$, C. Lebel ${ }^{93}$, M. Lechowski ${ }^{115}$, T. LeCompte ${ }^{5}$, F. Ledroit-Guillon ${ }^{55}$, H. Lee ${ }^{105}$, J.S.H. Lee ${ }^{149}$, S.C. Lee ${ }^{150}$, M. Lefebvre ${ }^{168}$, M. Legendre ${ }^{136}$, A. Leger ${ }^{49}$, B.C. LeGeyt ${ }^{120}$, F. Legger ${ }^{98}$, C. Leggett $^{14}$, M. Lehmacher ${ }^{20}$, G. Lehmann Miotto ${ }^{29}$, M. Lehto ${ }^{139}$, X. Lei ${ }^{6}$, R. Leitner ${ }^{126}$, D. Lellouch ${ }^{170}$, J. Lellouch ${ }^{78}$, M. Leltchouk ${ }^{34}$, V. Lendermann ${ }^{58 a}$, K.J.C. Leney ${ }^{73}$, T. Lenz ${ }^{173}$, G. Lenzen ${ }^{173}$, B. Lenzi ${ }^{136}$, K. Leonhardt ${ }^{43}$, J. Lepidis ${ }^{173}$, C. Leroy ${ }^{93}$, J-R. Lessard ${ }^{168}$, J. Lesser ${ }^{145 a}$, C.G. Lester ${ }^{27}$, A. Leung Fook Cheong ${ }^{171}$, J. Levêque ${ }^{83}$, D. Levin ${ }^{87}$, L.J. Levinson ${ }^{170}$, M.S. Levitski ${ }^{128}$, S. Levonian ${ }^{41}$, M. Lewandowska ${ }^{21}$, M. Leyton ${ }^{15}, \mathrm{H}^{2} \mathrm{Li}^{171}, \mathrm{~S} . \mathrm{Li}^{41}$, X. Li $^{87}$, Z. Liang ${ }^{39}$, Z. Liang ${ }^{150, p}$, B. Liberti ${ }^{133 a}$, P. Lichard ${ }^{29}$, M. Lichtnecker ${ }^{98}$, K. Lie ${ }^{164}$, W. Liebig ${ }^{105}$, R. Lifshitz ${ }^{151}$, J.N. Lilley ${ }^{17}$, H. Lim ${ }^{5}$, A. Limosani ${ }^{86}$, M. Limper ${ }^{63}$, S.C. Lin $^{150}$, F. Linde ${ }^{105}$, J.T. Linnemann ${ }^{88}$, E. Lipeles ${ }^{120}$, L. Lipinsky ${ }^{125}$, A. Lipniacka ${ }^{13}$, T.M. Liss ${ }^{164}$, D. Lissauer ${ }^{24}$, A. Lister ${ }^{49}$, A.M. Litke ${ }^{137}$, C. Liu ${ }^{28}$,

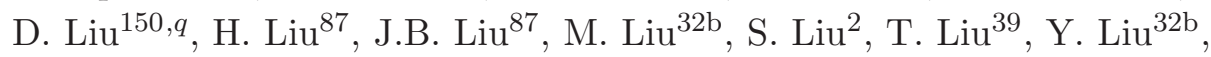
M. Livan ${ }^{119 a, 119 b}$, A. Lleres ${ }^{55}$, S.L. Lloyd ${ }^{75}$, F. Lobkowicz ${ }^{24, *}$, E. Lobodzinska ${ }^{41}$, P. Loch ${ }^{6}$, W.S. Lockman ${ }^{137}$, S. Lockwitz ${ }^{174}$, T. Loddenkoetter ${ }^{20}$, F.K. Loebinger ${ }^{82}$, A. Loginov ${ }^{174}$, C.W. Loh ${ }^{167}$, T. Lohse ${ }^{15}$, K. Lohwasser ${ }^{48}$, M. Lokajicek ${ }^{125}$, J. Loken ${ }^{118}$, R.E. Long ${ }^{71}$, L. Lopes ${ }^{124 a}$, D. Lopez Mateos ${ }^{34, k}$, M. Losada ${ }^{161}$, P. Loscutoff ${ }^{14}$, M.J. Losty ${ }^{158 a}$,

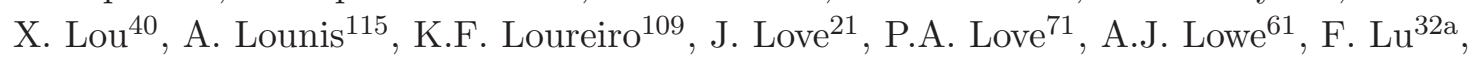
J. Lu ${ }^{2}$, L. Lu ${ }^{39}$, H.J. Lubatti138, C. Luci'132a,132b ${ }^{138}$ A. Lucotte ${ }^{55}$, A. Ludwig ${ }^{43}$, D. Ludwig ${ }^{41}$, I. Ludwig ${ }^{48}$, J. Ludwig ${ }^{48}$, F. Luehring ${ }^{61}$, G. Luijckx ${ }^{105}$, L. Luisa ${ }^{163 a, 163 c}$, D. Lumb ${ }^{48}$, L. Luminari ${ }^{132 a}$, E. Lund ${ }^{117}$, B. Lund-Jensen ${ }^{146}$, B. Lundberg ${ }^{79}$, J. Lundberg ${ }^{29}$, J. Lundquist ${ }^{35}$, A. Lupi ${ }^{122 a, 122 b}$, G. Lutz ${ }^{99}$, D. Lynn ${ }^{24}$, J. Lynn ${ }^{118}$, J. Lys ${ }^{14}$, E. Lytken ${ }^{79}$, H. $\mathrm{Ma}^{24}$, L.L. Ma ${ }^{171}$, M. Maaßen ${ }^{48}$, J.A. Macana Goia ${ }^{93}$, G. Maccarrone ${ }^{47}$,

A. Macchiolo ${ }^{99}$, B. Maček ${ }^{74}$, J. Machado Miguens ${ }^{124 a}$, D. Macina ${ }^{49}$, R. Mackeprang ${ }^{35}$, D. MacQueen ${ }^{2}$, R.J. Madaras ${ }^{14}$, W.F. Mader ${ }^{43}$, R. Maenner ${ }^{58 c}$, T. Maeno ${ }^{24}$, P. Mättig ${ }^{173}$, S. Mättig ${ }^{41}$, P.J. Magalhaes Martins ${ }^{124 a}$, E. Magradze ${ }^{51}$, C.A. Magrath ${ }^{104}$, Y. Mahalalel ${ }^{152}$, K. Mahboubi ${ }^{48}$, A. Mahmood ${ }^{1}$, G. Mahout ${ }^{17}$, C. Maiani132a,132b, C. Maidantchik ${ }^{23 a}$, A. Maio ${ }^{124 a}$, G.M. Mair ${ }^{62}$, S. Majewski ${ }^{24}$, Y. Makida ${ }^{66}$, M. Makouski ${ }^{128}$, N. Makovec ${ }^{115}$, P. Mal ${ }^{6}$, Pa. Malecki ${ }^{38}$, P. Malecki ${ }^{38}$, V.P. Maleev ${ }^{121}$, F. Malek ${ }^{55}$, U. Mallik ${ }^{63}$, D. Malon ${ }^{5}$, S. Maltezos ${ }^{9}$, V. Malyshev ${ }^{107}$, S. Malyukov ${ }^{65}$, M. Mambelli ${ }^{30}$, R. Mameghani ${ }^{98}$, J. Mamuzic ${ }^{41}$, A. Manabe ${ }^{66}$, A. Manara ${ }^{61}$, G. Manca ${ }^{73}$, L. Mandelli ${ }^{89 a}$, I. Mandić ${ }^{74}$, R. Mandrysch ${ }^{15}$, J. Maneira ${ }^{124 a}$, P.S. Mangeard ${ }^{88}$, M. Mangin-Brinet ${ }^{49}$, I.D. Manjavidze ${ }^{65}$, A. Mann ${ }^{54}$, W.A. Mann ${ }^{160}$, P.M. Manning ${ }^{137}$, A. Manousakis-Katsikakis ${ }^{8}$, B. Mansoulie ${ }^{136}$, A. Manz ${ }^{99}$, A. Mapelli ${ }^{29}$, L. Mapelli ${ }^{29}$, L. March ${ }^{80}$, J.F. Marchand ${ }^{4}$, F. Marchese ${ }^{133 a, 133 b}$, M. Marchesotti ${ }^{29}$, G. Marchiori ${ }^{78}$, M. Marcisovsky ${ }^{125}$, A. Marin ${ }^{21, *}$, C.P. Marino ${ }^{61}$, F. Marroquim ${ }^{23 a}$, R. Marshall ${ }^{82}$, Z. Marshall ${ }^{34, k}$, F.K. Martens ${ }^{157}$, S. Marti-Garcia ${ }^{166}$, A.J. Martin ${ }^{75}$, A.J. Martin ${ }^{174}$,

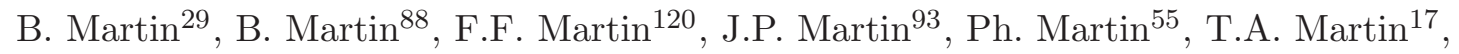


B. Martin dit Latour ${ }^{49}$, M. Martinez ${ }^{11}$, V. Martinez Outschoorn ${ }^{57}$, A. Martini ${ }^{47}$, V. Martynenko 158b , A.C. Martyniuk ${ }^{82}$, F. Marzano ${ }^{132 a}$, A. Marzin ${ }^{136}$, L. Masetti ${ }^{81}$, T. Mashimo ${ }^{154}$, R. Mashinistov ${ }^{96}$, J. Masik ${ }^{82}$, A.L. Maslennikov ${ }^{107}$, M. Maß32 $^{42}$, I. Massa ${ }^{19 a, 19 b}$, G. Massaro ${ }^{105}$, N. Massol ${ }^{4}$, A. Mastroberardino ${ }^{36 a, 36 b}$, T. Masubuchi ${ }^{154}$, M. Mathes ${ }^{20}$, P. Matricon ${ }^{115}$, H. Matsumoto ${ }^{154}$, H. Matsunaga ${ }^{154}$, T. Matsushita ${ }^{67}$, C. Mattravers ${ }^{118, r}$, J.M. Maugain ${ }^{29}$, S.J. Maxfield ${ }^{73}$, E.N. May ${ }^{5}$, J.K. Mayer ${ }^{157}$, A. Mayne ${ }^{139}$, R. Mazini ${ }^{150}$, M. Mazur $^{48}$, M. Mazzanti ${ }^{89 a}$, E. Mazzoni ${ }^{122 a, 122 b}$, J. Mc Donald ${ }^{85}$, S.P. Mc Kee ${ }^{87}$, A. McCarn ${ }^{164}$, R.L. McCarthy ${ }^{147}$, N.A. McCubbin ${ }^{129}$, K.W. McFarlane ${ }^{56}$, S. McGarvie ${ }^{76}$, H. McGlone ${ }^{53}$, G. Mchedlidze ${ }^{51}$, R.A. McLaren ${ }^{29}$, S.J. McMahon ${ }^{129}$, T.R. McMahon ${ }^{76}$, T.J. McMahon ${ }^{17}$, R.A. McPherson ${ }^{168, g}$, A. Meade ${ }^{84}$, J. Mechnich ${ }^{105}$, M. Mechtel ${ }^{173}$, M. Medinnis ${ }^{41}$, R. Meera-Lebbai ${ }^{111}$, T.M. Meguro ${ }^{116}$, R. Mehdiyev ${ }^{93}$, S. Mehlhase ${ }^{41}$, A. Mehta ${ }^{73}$, K. Meier ${ }^{58 a}$, J. Meinhardt ${ }^{48}$, B. Meirose ${ }^{48}$, C. Melachrinos ${ }^{30}$, B.R. Mellado Garcia ${ }^{171}$, L. Mendoza Navas ${ }^{161}$, Z. Meng150,s, S. Menke ${ }^{99}$, C. Menot ${ }^{29}$, E. Meoni ${ }^{11}$, D. Merkl ${ }^{98}$, P. Mermod $^{118}$, L. Merola ${ }^{102 a, 102 b}$, C. Meroni ${ }^{89 a}$, F.S. Merritt ${ }^{30}$, A.M. Messina ${ }^{29}$, I. Messmer ${ }^{48}$, J. Metcalfe ${ }^{103}$, A.S. Mete ${ }^{64}$, S. Meuser ${ }^{20}$, J-P. Meyer ${ }^{136}$, J. Meyer ${ }^{172}$, J. Meyer ${ }^{54}$, T.C. Meyer ${ }^{29}$, W.T. Meyer ${ }^{64}$,

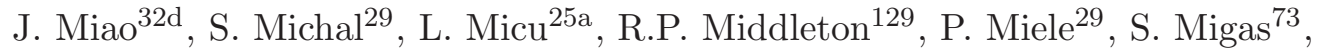

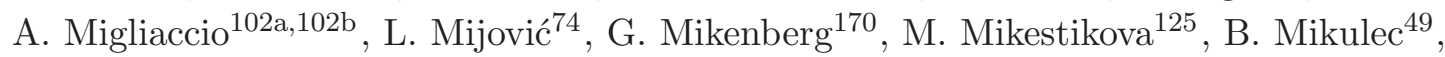
M. Mikuž ${ }^{74}$, D.W. Miller ${ }^{143}$, R.J. Miller ${ }^{88}$, W.J. Mills ${ }^{167}$, C.M. Mills ${ }^{57}$, A. Milov ${ }^{170}$, D.A. Milstead ${ }^{145 a, 145 b}$, D. Milstein ${ }^{170}$, S. Mima ${ }^{110}$, A.A. Minaenko ${ }^{128}$, M. Miñano ${ }^{166}$, I.A. Minashvili ${ }^{65}$, A.I. Mincer ${ }^{108}$, B. Mindur ${ }^{37}$, M. Mineev ${ }^{65}$, Y. Ming ${ }^{130}$, L.M. Mir ${ }^{11}$, G. Mirabelli ${ }^{132 a}$, L. Miralles Verge ${ }^{11}$, S. Misawa ${ }^{24}$, S. Miscetti $^{47}$, A. Misiejuk $^{76}$, A. Mitra ${ }^{118}$, J. Mitrevski ${ }^{137}$, G.Y. Mitrofanov ${ }^{128}$, V.A. Mitsou ${ }^{166}$, S. Mitsui ${ }^{159}$, P.S. Miyagawa ${ }^{82}$, K. Miyazaki ${ }^{67}$, J.U. Mjörnmark ${ }^{79}$, D. Mladenov ${ }^{22}$, T. Moa ${ }^{145 a, 145 b}$, M. Moch ${ }^{132 a, 132 b}$, P. Mockett ${ }^{138}$, S. Moed ${ }^{57}$, V. Moeller ${ }^{27}$, K. Mönig ${ }^{41}$, N. Möser ${ }^{20}$, B. Mohn ${ }^{13}$, W. Mohr ${ }^{48}$, S. Mohrdieck-Möck ${ }^{99}$, A.M. Moisseev ${ }^{128, *}$, R. Moles-Valls ${ }^{166}$, J. Molina-Perez ${ }^{29}$, L. Moneta ${ }^{49}$, J. Monk ${ }^{77}$, E. Monnier ${ }^{83}$, S. Montesano ${ }^{89 a, 89 b}$, F. Monticelli ${ }^{70}$, R.W. Moore ${ }^{2}$, T.B. Moore ${ }^{84}$, G.F. Moorhead ${ }^{86}$, C. Mora Herrera ${ }^{49}$, A. Moraes ${ }^{53}$, A. Morais ${ }^{124 a}$, J. Morel ${ }^{54}$, G. Morello ${ }^{36 a, 36 b}$, D. Moreno ${ }^{81}$, M. Moreno

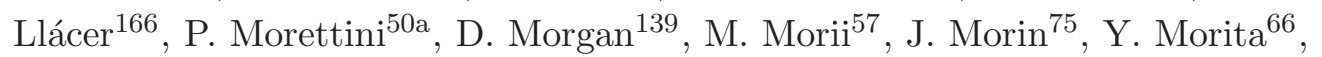
A.K. Morley ${ }^{86}$, G. Mornacchi' ${ }^{29}$, M-C. Morone ${ }^{49}$, S.V. Morozov ${ }^{96}$, J.D. Morris ${ }^{75}$, H.G. Moser ${ }^{99}$, M. Mosidze ${ }^{51}$, J. Moss ${ }^{109}$, A. Moszczynski ${ }^{38}$, R. Mount ${ }^{143}$, E. Mountricha ${ }^{136}$, S.V. Mouraviev ${ }^{94, *}$, T.H. Moye ${ }^{17}$, E.J.W. Moyse ${ }^{84}$, M. Mudrinic ${ }^{12 b}$, F. Mueller ${ }^{58 \mathrm{a}}$, J. Mueller ${ }^{123}$, K. Mueller ${ }^{20}$, T.A. Müller ${ }^{98}$, D. Muenstermann ${ }^{42}$, A. Muijs ${ }^{105}$, A. Muir ${ }^{167}$, A. Munar ${ }^{120}$, Y. Munwes ${ }^{152}$, K. Murakami ${ }^{66}$, R. Murillo Garcia ${ }^{162}$, W.J. Murray ${ }^{129}$, I. Mussche ${ }^{105}$, E. Musto ${ }^{102 a, 102 b}$, A.G. Myagkov ${ }^{128}$, M. Myska ${ }^{125}$, J. Nadal ${ }^{11}$, K. Nagai ${ }^{159}$, K. Nagano ${ }^{66}$, Y. Nagasaka ${ }^{60}$,

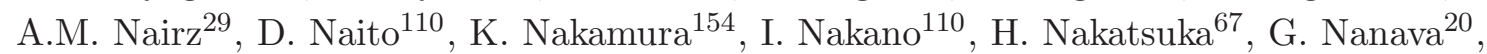
A. Napier ${ }^{160}$, M. Nash ${ }^{77, t}$, I. Nasteva ${ }^{82}$, N.R. Nation ${ }^{21}$, T. Nattermann ${ }^{20}$, T. Naumann ${ }^{41}$, F. Nauyock ${ }^{82}$, G. Navarro ${ }^{161}$, S.K. Nderitu ${ }^{20}$, H.A. Neal ${ }^{87}$, E. Nebot ${ }^{80}$, P. Nechaeva ${ }^{94}$, A. Negri ${ }^{119 a, 119 b}$, G. Negri ${ }^{29}$, S. Negroni ${ }^{34}$, A. Nelson ${ }^{64}$, S. Nelson ${ }^{143}$, T.K. Nelson ${ }^{143}$, S. Nemecek ${ }^{125}$, P. Nemethy ${ }^{108}$, A.A. Nepomuceno ${ }^{23 a}$, M. Nessi ${ }^{29}$, S.Y. Nesterov ${ }^{121}$, M.S. Neubauer ${ }^{164}$, L. Neukermans ${ }^{4}$, A. Neusiedl ${ }^{81}$, R.M. Neves ${ }^{108}$, P. Nevski ${ }^{24}$, 
F.M. Newcomer ${ }^{120}$, C. Nicholson ${ }^{53}$, R.B. Nickerson ${ }^{118}$, R. Nicolaidou ${ }^{136}$, L. Nicolas ${ }^{139}$, G. Nicoletti ${ }^{47}$, B. Nicquevert ${ }^{29}$, F. Niedercorn ${ }^{115}$, M. Niegl ${ }^{46, *}$, J. Nielsen ${ }^{137}$, T. Niinikoski ${ }^{29}$, A. Nikiforov ${ }^{15}$, K. Nikolaev ${ }^{65}$, I. Nikolic-Audit ${ }^{78}$, K. Nikolopoulos ${ }^{8}$, H. Nilsen ${ }^{48}$, P. Nilsson ${ }^{7}$, A. Nisati132a, T. Nishiyama ${ }^{67}$, R. Nisius ${ }^{99}$, L. Nodulman $^{5}$, M. Nomachi ${ }^{116}$, I. Nomidis ${ }^{153}$, H. Nomoto ${ }^{154}$, M. Nordberg ${ }^{29}$, B. Nordkvist ${ }^{145 a, 145 b}$, O. Norniella Francisco ${ }^{11}$, P.R. Norton ${ }^{129}$, D. Notz ${ }^{41}$, J. Novakova ${ }^{126}$, M. Nozaki ${ }^{66}$, M. Nožička ${ }^{41}$, I.M. Nugent ${ }^{158 a}$, A.-E. Nuncio-Quiroz ${ }^{20}$, G. Nunes Hanninger ${ }^{20}$, T. Nunnemann ${ }^{98}$, E. Nurse ${ }^{77}$, T. Nyman ${ }^{29}$, S.W. O'Neale ${ }^{17, *}$, D.C. O'Neil ${ }^{142}$, V. O'Shea ${ }^{53}$, F.G. Oakham ${ }^{28, c}$, H. Oberlack ${ }^{99}$, M. Obermaier ${ }^{98}$, P. Oberson ${ }^{132 a, 132 b}$,

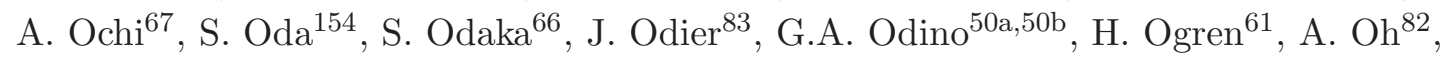
S.H. $\mathrm{Oh}^{44}$, C.C. Ohm ${ }^{145 a, 145 b}$, T. Ohshima ${ }^{101}$, H. Ohshita ${ }^{140}$, T.K. Ohska ${ }^{66}$, T. Ohsugi ${ }^{59}$, S. Okada ${ }^{67}$, H. Okawa ${ }^{162}$, Y. Okumura ${ }^{101}$, T. Okuyama ${ }^{154}$, M. Olcese ${ }^{50 a}$, A.G. Olchevski ${ }^{65}$, M. Oliveira ${ }^{124 a}$, D. Oliveira Damazio ${ }^{24}$, C. Oliver ${ }^{80}$, J. Oliver ${ }^{57}$, E. Oliver Garcia ${ }^{166}$, D. Olivito ${ }^{120}$, M. Olivo Gomez ${ }^{99}$, A. Olszewski ${ }^{38}$, J. Olszowska ${ }^{38}$, C. Omachi ${ }^{67, u}$, A. Onea ${ }^{29}$, A. Onofre ${ }^{124 a}$, P.U.E. Onyisi ${ }^{30}$, C.J. Oram ${ }^{158 a}$, G. Ordonez ${ }^{104}$, M.J. Oreglia ${ }^{30}$, F. Orellana ${ }^{49}$, Y. Oren ${ }^{152}$, D. Orestano ${ }^{134 a, 134 b}$, I. Orlov ${ }^{107}$,

C. Oropeza Barrera ${ }^{53}$, R.S. Orr ${ }^{157}$, E.O. Ortega ${ }^{130}$, B. Osculati ${ }^{50 a, 50 b}$, R. Ospanov ${ }^{120}$,

C. Osuna ${ }^{11}$, G. Otero y Garzon ${ }^{26}$, J.P Ottersbach ${ }^{105}$, B. Ottewell ${ }^{118}$, F. Ould-Saada ${ }^{117}$, A. Ouraou ${ }^{136}$, Q. Ouyang ${ }^{32 a}$, M. Owen ${ }^{82}$, S. Owen ${ }^{139}$, A Oyarzun $^{31 b}$, O.K. Øye ${ }^{13}$, V.E. Ozcan ${ }^{77}$, K. Ozone ${ }^{66}$, N. Ozturk ${ }^{7}$, A. Pacheco Pages ${ }^{11}$, C. Padilla Aranda ${ }^{11}$, E. Paganis ${ }^{139}$, C. Pahl ${ }^{63}$, F. Paige ${ }^{24}$, K. Pajchel ${ }^{117}$, S. Palestini ${ }^{29}$, J. Palla ${ }^{29}$, D. Pallin ${ }^{33}$, A. Palma ${ }^{124 a}$, J.D. Palmer ${ }^{17}$, M.J. Palmer ${ }^{27}$, Y.B. Pan $^{171}$, E. Panagiotopoulou ${ }^{9}$, B. Panes ${ }^{31 a}$, N. Panikashvili ${ }^{87}$, V.N. Panin ${ }^{107, *}$, S. Panitkin ${ }^{24}$, D. Pantea ${ }^{25 a}$, M. Panuskova ${ }^{125}$, V. Paolone ${ }^{123}$, A. Paoloni ${ }^{133 a, 133 b}$, I. Papadopoulos ${ }^{29}$, Th.D. Papadopoulou ${ }^{9}$, S.J. Park ${ }^{54}$, W. Park $^{24, v}$, M.A. Parker ${ }^{27}$, S.I. Parker ${ }^{14}$, F. Parodi ${ }^{50 a, 50 b}$, J.A. Parsons ${ }^{34}$, U. Parzefall ${ }^{48}$, E. Pasqualucci ${ }^{132 a}$, A. Passeri $^{134 a}$, F. Pastore ${ }^{134 a, 134 b}$, Fr. Pastore ${ }^{29}$, G. Pásztor ${ }^{49, w}$, S. Pataraia $^{99}$, N. Patel ${ }^{149}$, J.R. Pater ${ }^{82}$, S. Patricelli ${ }^{102 a, 102 b}$, A. Patwa ${ }^{24}$, T. Pauly ${ }^{29}$, L.S. Peak ${ }^{149}$, M. Pecsy ${ }^{144 a}$, M.I. Pedraza Morales ${ }^{171}$, S.J.M. Peeters ${ }^{105}$, M. Peez ${ }^{80}$, S.V. Peleganchuk ${ }^{107}$, H. Peng ${ }^{171}$, R. Pengo ${ }^{29}$, A. Penson ${ }^{34}$, J. Penwell ${ }^{61}$, M. Perantoni ${ }^{23 a}$, K. Perez ${ }^{34, k}$, E. Perez Codina ${ }^{11}$, M.T. Pérez García-Estañ ${ }^{166}$, V. Perez Reale ${ }^{34}$, I. Peric ${ }^{20}$, L. Perini ${ }^{89 a, 89 b}$, H. Pernegger ${ }^{29}$, R. Perrino ${ }^{72 a}$, P. Perrodo ${ }^{4}$, S. Persembe ${ }^{3 a}$, P. Perus ${ }^{115}$, V.D. Peshekhonov ${ }^{65}$, E. Petereit ${ }^{5}$, O. Peters ${ }^{105}$, B.A. Petersen ${ }^{29}$, J. Petersen ${ }^{29}$, T.C. Petersen ${ }^{35}$, E. Petit ${ }^{83}$, C. Petridou ${ }^{153}$, E. Petrolo ${ }^{132 a}$, F. Petrucci ${ }^{134 a, 134 b}$, D Petschull ${ }^{41}$, M. Petteni ${ }^{142}$, R. Pezoa ${ }^{31 b}$, B. Pfeifer ${ }^{48}$, A. $\mathrm{Phan}^{86}$, A.W. Phillips ${ }^{27}$, G. Piacquadio ${ }^{29}$, E. Piccaro ${ }^{75}$, M. Piccinini ${ }^{19 a, 19 b}$, A. Pickford ${ }^{53}$, R. Piegaia ${ }^{26}$, J.E. Pilcher ${ }^{30}$, A.D. Pilkington ${ }^{82}$, J. Pina ${ }^{124 a}$, M. Pinamonti163a,163c , J.L. Pinfold ${ }^{2}$, J. Ping ${ }^{32 c}$, B. Pinto ${ }^{124 a}$, O. Pirotte ${ }^{29}$, C. Pizio ${ }^{89 a, 89 b}$, R. Placakyte ${ }^{41}$, M. Plamondon ${ }^{168}$, W.G. Plano ${ }^{82}$, M.-A. Pleier ${ }^{24}$, A.V. Pleskach ${ }^{128}$, A. Poblaguev ${ }^{174}$, S. Poddar ${ }^{58 a}$, F. Podlyski ${ }^{33}$, P. Poffenberger ${ }^{168}$, L. Poggioli ${ }^{115}$, M. Pohl ${ }^{49}$, F. Polci ${ }^{55}$, G. Polesello ${ }^{119 a}$, A. Policicchio ${ }^{138}$, A. Polini ${ }^{19 a}$, J. Poll ${ }^{75}$, V. Polychronakos ${ }^{24}$, D.M. Pomarede ${ }^{136}$, D. Pomeroy ${ }^{22}$, K. Pommès ${ }^{29}$, P. Ponsot ${ }^{136}$, L. Pontecorvo ${ }^{132 a}$, B.G. Pope ${ }^{88}$, G.A. Popeneciu ${ }^{25 a}$, R. Popescu ${ }^{24}$, D.S. Popovic ${ }^{12 \mathrm{a}}$, A. Poppleton ${ }^{29}$, J. Popule ${ }^{125}$, X. Portell Bueso ${ }^{48}$, R. Porter ${ }^{162}$, 
C. Posch $^{21}$, G.E. Pospelov ${ }^{99}$, P. Pospichal ${ }^{29}$, S. Pospisil ${ }^{127}$, M. Potekhin ${ }^{24}$, I.N. Potrap ${ }^{99}$, C.J. Potter ${ }^{148}$, C.T. Potter ${ }^{85}$, K.P. Potter ${ }^{82}$, G. Poulard ${ }^{29}$, J. Poveda ${ }^{171}$, R. Prabhu ${ }^{20}$, P. Pralavorio ${ }^{83}$, S. Prasad ${ }^{57}$, M. Prata ${ }^{119 a, 119 b}$, R. Pravahan ${ }^{7}$, K. Pretzl ${ }^{16}$, L. Pribyl ${ }^{29}$, D. Price ${ }^{61}$, L.E. Price $^{5}$, M.J. Price ${ }^{29}$, P.M. Prichard ${ }^{73}$, D. Prieur ${ }^{123}$, M. Primavera ${ }^{72 a}$, D. Primor ${ }^{29}$, K. Prokofiev ${ }^{29}$, F. Prokoshin ${ }^{31 b}$, S. Protopopescu ${ }^{24}$, J. Proudfoot ${ }^{5}$, X. Prudent ${ }^{43}$, H. Przysiezniak ${ }^{4}$, S. Psoroulas ${ }^{20}$, E. Ptacek ${ }^{114}$, C. Puigdengoles ${ }^{11}$, J. Purdham ${ }^{87}$, M. Purohit ${ }^{24, v}$, P. Puzo ${ }^{115}$, Y. Pylypchenko ${ }^{117}$, M. Qi $^{32 c}$, J. Qian ${ }^{87}$, W. Qian ${ }^{129}$, Z. Qian ${ }^{83}$, Z. Qin ${ }^{41}$, D. Qing ${ }^{150, x}$, A. Quadt ${ }^{54}$, D.R. Quarrie ${ }^{14}$, W.B. Quayle ${ }^{171}$, F. Quinonez ${ }^{31 a}$, M. Raas ${ }^{104}$, V. Radeka ${ }^{24}$, V. Radescu ${ }^{58 b}$, B. Radics ${ }^{20}$, T. Rador ${ }^{18 a}$, F. Ragusa ${ }^{89 a, 89 b}$, G. Rahal ${ }^{179}$, A.M. Rahimi ${ }^{109}$, D. Rahm ${ }^{24}$, C. Raine $^{53, *}$, B. Raith ${ }^{20}$, S. Rajagopalan ${ }^{24}$, S. Rajek ${ }^{42}$, M. Rammensee ${ }^{48}$, M. Rammes ${ }^{141}$, M. Ramstedt ${ }^{145 a, 145 b}$, P.N. Ratoff ${ }^{71}$, F. Rauscher ${ }^{98}$, E. Rauter ${ }^{99}$, M. Raymond ${ }^{29}$, A.L. $\operatorname{Read}^{117}$, D.M. Rebuzzi ${ }^{119 a, 119 b}$, A. Redelbach ${ }^{172}$, G. Redlinger ${ }^{24}$, R. Reece ${ }^{120}$, K. Reeves ${ }^{40}$, M. Rehak ${ }^{24}$, A. Reichold ${ }^{105}$, E. Reinherz-Aronis ${ }^{152}$, A Reinsch ${ }^{114}$, I. Reisinger ${ }^{42}$, D. Reljic ${ }^{12 a}$, C. Rembser ${ }^{29}$, Z.L. Ren ${ }^{150}$, P. Renkel ${ }^{39}$, B. Rensch ${ }^{35}$, S. Rescia ${ }^{24}$, M. Rescigno ${ }^{132 a}$, S. Resconi ${ }^{89 a}$, B. Resende ${ }^{136}$, E. Rezaie ${ }^{142}$, P. Reznicek ${ }^{126}$, R. Rezvani ${ }^{157}$, A. Richards ${ }^{77}$, R.A. Richards ${ }^{88}$, R. Richter ${ }^{99}$, E. Richter-Was ${ }^{38, y}$, M. Ridel ${ }^{78}$, S. Rieke ${ }^{81}$, M. Rijpstra ${ }^{105}$, M. Rijssenbeek ${ }^{147}$, A. Rimoldi ${ }^{119 a, 119 b}$, L. Rinaldi ${ }^{19 a}$, R.R. $\operatorname{Rios}^{39}$, C. Risler ${ }^{15}$, I. Riu ${ }^{11}$, G. Rivoltella ${ }^{89 a, 89 b}$, F. Rizatdinova ${ }^{112}$, E. Rizvi ${ }^{75}$, D.A. Roa Romero ${ }^{161}$, S.H. Robertson ${ }^{85, g}$, A. Robichaud-Veronneau ${ }^{49}$, S. Robins ${ }^{132 a, 132 b}$, D. Robinson ${ }^{27}$, JEM Robinson ${ }^{77}$, M. Robinson ${ }^{114}$, A. Robson ${ }^{53}$, J.G. Rocha de Lima ${ }^{106 a}$, C. Roda ${ }^{122 a, 122 b}$, D. Roda Dos Santos ${ }^{29}$, S. Rodier ${ }^{80}$, D. Rodriguez ${ }^{161}$, Y. Rodriguez Garcia ${ }^{15}$, S. Roe $^{29}$, O. Røhne ${ }^{117}$, V. Rojo ${ }^{1}$, S. Rolli ${ }^{160}$, A. Romaniouk ${ }^{96}$, V.M. Romanov ${ }^{65}$, G. Romeo ${ }^{26}$, D. Romero Maltrana ${ }^{31 a}$, L. Roos ${ }^{78}$, E. $\operatorname{Ros}^{166}$, S. Rosati ${ }^{138}$, F. Rosenbaum ${ }^{137}$, G.A. Rosenbaum ${ }^{157}$, E.I. Rosenberg ${ }^{64}$, L. Rosselet ${ }^{49}$, V. Rossetti ${ }^{11}$, L.P. Rossi ${ }^{50 a}$, L. Rossi ${ }^{89 a, 89 b}$, M. Rotaru ${ }^{25 a}$, J. Rothberg ${ }^{138}$, I. Rottländer ${ }^{20}$, D. Rousseau ${ }^{115}$, C.R. Royon ${ }^{136}$, A. Rozanov ${ }^{83}$, Y. Rozen ${ }^{151}$, X. Ruan ${ }^{115}$, B. Ruckert ${ }^{98}$, N. Ruckstuhl ${ }^{105}$, V.I. Rud ${ }^{97}$, G. Rudolph ${ }^{62}$, F. Rühr ${ }^{58 a}$, F. Ruggieri ${ }^{134 a}$, A. Ruiz-Martinez ${ }^{64}$, E. Rulikowska-Zarebska ${ }^{37}$, V. Rumiantsev ${ }^{91, *}$, L. Rumyantsev $^{65}$, K. Runge ${ }^{48}$, O. Runolfsson ${ }^{20}$, Z. Rurikova ${ }^{48}$, N.A. Rusakovich ${ }^{65}$, D.R. Rust ${ }^{61}$, J.P. Rutherfoord ${ }^{6}$, C. Ruwiedel ${ }^{20}$, P. Ruzicka ${ }^{125}$, Y.F. Ryabov ${ }^{121}$, V. Ryadovikov ${ }^{128}$, P. Ryan ${ }^{88}$, G. Rybkin ${ }^{115}$, S. Rzaeva ${ }^{10}$, A.F. Saavedra ${ }^{149}$, H.F-W. Sadrozinski ${ }^{137}$, R. Sadykov ${ }^{65}$, F. Safai Tehrani132a,132b ${ }^{13}$ H. Sakamoto ${ }^{154}$, P. Sala ${ }^{89 a}$, G. Salamanna ${ }^{105}$, A. Salamon ${ }^{133 a}$, M.S. Saleem ${ }^{111}$, D. Salihagic ${ }^{99}$, A. Salnikov ${ }^{143}$, J. Salt ${ }^{166}$, O. Saltó Bauza $^{11}$, B.M. Salvachua Ferrando ${ }^{5}$, D. Salvatore ${ }^{36 a, 36 b}$, F. Salvatore ${ }^{148}$, A. Salvucci ${ }^{47}$, A. Salzburger ${ }^{29}$, D. Sampsonidis ${ }^{153}$, B.H. Samset ${ }^{117}$, C.A. Sánchez Sánchez ${ }^{11}$, H. Sandaker ${ }^{13}$, H.G. Sander ${ }^{81}$, M.P. Sanders ${ }^{98}$, M. Sandhoff ${ }^{173}$, P. Sandhu ${ }^{157}$, R. Sandstroem ${ }^{105}$, S. Sandvoss ${ }^{173}$, D.P.C. Sankey ${ }^{129}$, B. Sanny ${ }^{173}$, A. Sansoni ${ }^{47}$, C. Santamarina Rios ${ }^{85}$, C. Santoni ${ }^{33}$, R. Santonico ${ }^{133 a, 133 b}$, J.G. Saraiva ${ }^{124 a}$, T. Sarangi ${ }^{171}$, E. Sarkisyan-Grinbaum ${ }^{7}$, F. Sarri ${ }^{122 a, 122 b}$, O. Sasaki ${ }^{66}$, T. Sasaki ${ }^{66}$, N. Sasao ${ }^{68}$, I. Satsounkevitch ${ }^{90}$, G. Sauvage ${ }^{4, *}$, P. Savard ${ }^{157, c}$, A.Y. Savine ${ }^{6}$, V. Savinov ${ }^{123}$, A. Savoy-Navarro ${ }^{78}$, P. Savva ${ }^{9}$, L. Sawyer ${ }^{24, h}$, D.H. Saxon ${ }^{53}$, L.P. Says ${ }^{33}$, C. Sbarra ${ }^{19 a, 19 b}$, A. Sbrizzi ${ }^{19 a, 19 b}$, D.A. Scannicchio ${ }^{29}$, J. Schaarschmidt ${ }^{43}$, P. Schacht ${ }^{99}$, 
U. Schäfer ${ }^{81}$, S. Schaetzel ${ }^{58 b}$, A.C. Schaffer ${ }^{115}$, D. Schaile ${ }^{98}$, M. Schaller ${ }^{29}$, R.D. Schamberger ${ }^{147}$, A.G. Schamov ${ }^{107}$, V. Scharf ${ }^{58 a}$, V.A. Schegelsky ${ }^{121}$, D. Scheirich ${ }^{87}$, M. Schernau ${ }^{162}$, M.I. Scherzer ${ }^{14}$, C. Schiavi ${ }^{50 a, 50 b}$, J. Schieck ${ }^{99}$, M. Schioppa ${ }^{36 a, 36 b}$, G. Schlager ${ }^{29}$, S. Schlenker ${ }^{29}$, J.L. Schlereth ${ }^{5}$, E. Schmidt ${ }^{48}$, M.P. Schmidt ${ }^{174, *}$, K. Schmieden ${ }^{20}$, C. Schmitt ${ }^{81}$, M. Schmitz ${ }^{20}$, R.C. Scholte ${ }^{105}$, A. Schönig ${ }^{58 b}$, M. Schott ${ }^{29}$, D. Schouten ${ }^{142}$, J. Schovancova ${ }^{125}$, M. Schram ${ }^{85}$, A. Schreiner ${ }^{63}$, A. Schricker ${ }^{22}$, C. Schroeder ${ }^{81}$, N. Schroer ${ }^{58 c}$, M. Schroers ${ }^{173}$, D. Schroff ${ }^{48}$, S. Schuh ${ }^{29}$, G. Schuler ${ }^{29}$, J. Schultes ${ }^{173}$, H.-C. Schultz-Coulon ${ }^{58 a}$, J.W. Schumacher ${ }^{43}$, M. Schumacher ${ }^{48}$, B.A. Schumm ${ }^{137}$, Ph. Schune ${ }^{136}$, C. Schwanenberger ${ }^{82}$, A. Schwartzman ${ }^{143}$, D. Schweiger ${ }^{29}$, Ph. Schwemling ${ }^{78}$, R. Schwienhorst ${ }^{88}$, R. Schwierz ${ }^{43}$, J. Schwindling ${ }^{136}$, W.G. Scott ${ }^{129}$, J. Searcy ${ }^{114}$, E. Sedykh ${ }^{121}$, E. Segura ${ }^{11}$, S.C. Seidel ${ }^{103}$, A. Seiden ${ }^{137}$, F. Seifert ${ }^{43}$, J.M. Seixas ${ }^{23 a}$, G. Sekhniaidze ${ }^{102 a}$, D.M. Seliverstov ${ }^{121}$, B. Sellden ${ }^{145 a}$, M. Seman ${ }^{144 b}$, N. Semprini-Cesari ${ }^{19 a, 19 b}$, C. Serfon ${ }^{98}$, L. Serin ${ }^{115}$, R. Seuster ${ }^{99}$, H. Severini ${ }^{111}$, M.E. Sevior ${ }^{86}$, A. Sfyrla ${ }^{164}$, E. Shabalina ${ }^{54}$, T.P. $\operatorname{Shah}^{129}$, M. Shamim ${ }^{114}$, L.Y. Shan ${ }^{32 a}$, J.T. Shank ${ }^{21}$, Q.T. Shao ${ }^{86}$, M. Shapiro ${ }^{14}$, P.B. Shatalov ${ }^{95}$, L. Shaver ${ }^{6}$, C. Shaw ${ }^{53}$, K. Shaw ${ }^{139}$, D. Sherman ${ }^{29}$, P. Sherwood ${ }^{77}$, A. Shibata ${ }^{108}$, P. Shield ${ }^{118}$, M. Shimojima ${ }^{100}$, T. Shin ${ }^{56}$, A. Shmeleva ${ }^{94}$, M.J. Shochet ${ }^{30}$, M.A. Shupe ${ }^{6}$, P. Sicho ${ }^{125}$, A. Sidoti ${ }^{15}$, A. Siebel ${ }^{173}$, M. Siebel ${ }^{29}$, F Siegert ${ }^{77}$, J. Siegrist ${ }^{14}$, Dj. Sijacki ${ }^{12 a}$, O. Silbert ${ }^{170}$, J. Silva ${ }^{124 a}$, Y. Silver ${ }^{152}$, D. Silverstein ${ }^{143}$, S.B. Silverstein ${ }^{145 a}$, V. Simak ${ }^{127}$, Lj. Simic ${ }^{12 a}$, S. Simion ${ }^{115}$, B. Simmons ${ }^{77}$, M. Simonyan ${ }^{35}$, P. Sinervo ${ }^{157}$, N.B. Sinev ${ }^{114}$, V. Sipica ${ }^{141}$, G. Siragusa ${ }^{81}$, A.N. Sisakyan ${ }^{65, *}$, S.Yu. Sivoklokov ${ }^{97}$, J. Sjoelin ${ }^{145 a, 145 b}$, T.B. Sjursen ${ }^{13}$, K. Skovpen ${ }^{107}$, P. Skubic ${ }^{111}$, N. Skvorodnev ${ }^{22}$, M. Slater ${ }^{17}$, P. Slattery ${ }^{24, z}$, T. Slavicek ${ }^{127}$, K. Sliwa ${ }^{160}$, T.J. Sloan ${ }^{71}$, J. Sloper ${ }^{29}$, T. Sluka ${ }^{125}$, V. Smakhtin ${ }^{170}$, A. Small ${ }^{71}$, S.Yu. Smirnov ${ }^{96}$, Y. Smirnov ${ }^{24}$, L.N. Smirnova ${ }^{97}$, O. Smirnova ${ }^{79}$, B.C. Smith ${ }^{57}$, D. Smith ${ }^{143}$, K.M. Smith ${ }^{53}$, M. Smizanska ${ }^{71}$, K. Smolek ${ }^{127}$, A.A. Snesarev ${ }^{94}$, S.W. Snow ${ }^{82}$, J. Snow ${ }^{111}$, J. Snuverink ${ }^{105}$, S. Snyder ${ }^{24}$, M. Soares ${ }^{124 a}$, R. Sobie ${ }^{168, g}$, J. Sodomka ${ }^{127}$, A. Soffer ${ }^{152}$, C.A. Solans ${ }^{166}$, M. Solar ${ }^{127}$, J. Solc ${ }^{127}$, E. Solfaroli Camillocci ${ }^{132 a, 132 b}$, A.A. Solodkov ${ }^{128}$, O.V. Solovyanov ${ }^{128}$, R. Soluk ${ }^{2}$, J. Sondericker ${ }^{24}$, V. Sopko ${ }^{127}$, B. Sopko ${ }^{127}$, M. Sorbi ${ }^{89 a, 89 b}$, M. Sosebee ${ }^{7}$, A. Soukharev ${ }^{107}$, S. Spagnolo ${ }^{72 a, 72 b}$, F. Spanò ${ }^{34}$, P. Speckmayer ${ }^{29}$, E. Spencer ${ }^{137}$, R. Spighi ${ }^{19 a}$, G. Spigo ${ }^{29}$, F. Spila ${ }^{132 a, 132 b}$, E. Spiriti ${ }^{134 a}$, R. Spiwoks ${ }^{29}$, L. Spogli134a,134b, M. Spousta ${ }^{126}$, T. Spreitzer ${ }^{142}$, B. Spurlock ${ }^{7}$, R.D. St. Denis ${ }^{53}$, T. Stahl ${ }^{141}$, J. Stahlman ${ }^{120}$, R. Stamen ${ }^{58 a}$, S.N. Stancu ${ }^{162}$, E. Stanecka ${ }^{29}$, R.W. Stanek ${ }^{5}$, C. Stanescu ${ }^{134 a}$, S. Stapnes ${ }^{117}$, E.A. Starchenko ${ }^{128}$, J. Stark ${ }^{55}$, P. Staroba ${ }^{125}$, P. Starovoitov ${ }^{91}$, J. Stastny ${ }^{125}$, A. Staude ${ }^{98}$, P. Stavina ${ }^{144 a, *}$, G. Stavropoulos ${ }^{14}$, G. Steele ${ }^{53}$, E. Stefanidis ${ }^{77}$, P. Steinbach ${ }^{43}$, P. Steinberg ${ }^{24}$, I. Stekl ${ }^{127}$, B. Stelzer ${ }^{142}$, H.J. Stelzer ${ }^{41}$, O. Stelzer-Chilton ${ }^{158 a}$, H. Stenzel ${ }^{52}$, K. Stevenson ${ }^{75}$, G.A. Stewart ${ }^{53}$, T.D. Stewart ${ }^{142}$, W. Stiller ${ }^{99}$, T. Stockmanns ${ }^{20}$, M.C. Stockton ${ }^{29}$, M. Stodulski ${ }^{38}$, K. Stoerig ${ }^{48}$, G. Stoicea ${ }^{25 a}$, S. Stonjek ${ }^{99}$, P. Strachota ${ }^{126}$, A.R. Stradling ${ }^{7}$, A. Straessner ${ }^{43}$, J. Strandberg ${ }^{87}$, S. Strandberg ${ }^{14}$, A. Strandlie ${ }^{117}$, M. Strang ${ }^{109}$, M. Strauss ${ }^{111}$, P. Strizenec ${ }^{144 b}$, R. Ströhmer ${ }^{172}$, D.M. Strom ${ }^{114}$, J.A. Strong ${ }^{76, *}$, R. Stroynowski ${ }^{39}$, J. Strube ${ }^{129}$, B. Stugu ${ }^{13}$, I. Stumer ${ }^{24, *}$ P. Sturm ${ }^{173}$, D.A. Soh ${ }^{150, a a}$, D. $\mathrm{Su}^{143}$, S. Subramania ${ }^{61}$, Y. Sugaya ${ }^{116}$, T. Sugimoto ${ }^{101}$, C. Suhr ${ }^{106 a}$, K. Suita ${ }^{67}$, 
M. Suk ${ }^{126}$, V.V. Sulin ${ }^{94}$, S. Sultansoy ${ }^{3 d}$, T. Sumida ${ }^{29}$, X.H. Sun ${ }^{32 d}$, J.E. Sundermann ${ }^{48}$, K. Suruliz ${ }^{163 a, 163 b}$, S. Sushkov ${ }^{11}$, G. Susinno ${ }^{36 a, 36 b}$, M.R. Sutton ${ }^{139}$, T. Suzuki ${ }^{154}$, Y. Suzuki ${ }^{66}$, Yu.M. Sviridov ${ }^{128}$, I. Sykora ${ }^{144 a}$, T. Sykora ${ }^{126}$, R.R. Szczygiel ${ }^{38}$, B. Szeless ${ }^{29}$, T. Szymocha ${ }^{38}$, J. Sánchez ${ }^{166}$, D. Ta ${ }^{20}$, S. Taboada Gameiro ${ }^{29}$, K. Tackmann ${ }^{29}$,

A. Taffard ${ }^{162}$, R. Tafirout ${ }^{158 a}$, A. Taga ${ }^{117}$, Y. Takahashi ${ }^{101}$, H. Takai ${ }^{24}$, R. Takashima ${ }^{69}$, H. Takeda ${ }^{67}$, T. Takeshita ${ }^{140}$, M. Talby ${ }^{83}$, A. Talyshev ${ }^{107}$, M.C. Tamsett ${ }^{76}$, J. Tanaka ${ }^{154}$, R. Tanaka ${ }^{115}$, S. Tanaka ${ }^{131}$, S. Tanaka ${ }^{66}$, Y. Tanaka ${ }^{100}$, K. Tani ${ }^{67}$, G.P. Tappern ${ }^{29}$, S. Tapprogge ${ }^{81}$, D. Tardif ${ }^{157}$, S. Tarem ${ }^{151}$, F. Tarrade ${ }^{24}$, G.F. Tartarelli ${ }^{89 a}$, P. Tas ${ }^{126}$, M. Tasevsky ${ }^{125}$, E. Tassi ${ }^{36 a, 36 b}$, M. Tatarkhanov ${ }^{14}$, Y. Tayalati ${ }^{135 c}$, C. Taylor ${ }^{77}$, F.E. Taylor ${ }^{92}$, G. Taylor ${ }^{137}$, G.N. Taylor ${ }^{86}$, R.P. Taylor ${ }^{168}$, W. Taylor ${ }^{158 b}$, M. Teixeira Dias Castanheira ${ }^{75}$, P. Teixeira-Dias ${ }^{76}$, H. Ten Kate ${ }^{29}$, P.K. Teng ${ }^{150}$, Y.D. Tennenbaum-Katan ${ }^{151}$, S. Terada ${ }^{66}$, K. Terashi ${ }^{154}$, J. Terron ${ }^{80}$, M. Terwort ${ }^{41, m}$, M. Testa ${ }^{47}$, R.J. Teuscher ${ }^{157, g}$, C.M. Tevlin ${ }^{82}$, J. Thadome ${ }^{173}$, J. Therhaag ${ }^{20}$, M. Thioye ${ }^{174}$, S. Thoma ${ }^{48}$, J.P. Thomas ${ }^{17}$, E.N. Thompson ${ }^{84}$, P.D. Thompson ${ }^{17}$, P.D. Thompson ${ }^{157}$, R.J. Thompson ${ }^{82}$, A.S. Thompson ${ }^{53}$, E. Thomson ${ }^{120}$, R.P. Thun ${ }^{87}$, T. Tic ${ }^{125}$, V.O. Tikhomirov ${ }^{94}$, Y.A. Tikhonov ${ }^{107}$, C.J.W.P. Timmermans ${ }^{104}$, P. Tipton ${ }^{174}$, F.J. Tique Aires Viegas ${ }^{29}$, S. Tisserant ${ }^{83}$, J. Tobias ${ }^{48}$, B. Toczek ${ }^{37}$, T. Todorov ${ }^{4}$, S. Todorova-Nova ${ }^{160}$, B. Toggerson ${ }^{162}$, J. Tojo ${ }^{66}$, S. Tokár ${ }^{144 a}$, K. Tokunaga ${ }^{67}$, K. Tokushuku ${ }^{66}$, K. Tollefson ${ }^{88}$, L. Tomasek ${ }^{125}$, M. Tomasek ${ }^{125}$, M. Tomoto ${ }^{101}$, D. Tompkins ${ }^{6}$, L. Tompkins ${ }^{14}$, K. Toms ${ }^{103}$, A. Tonazzo ${ }^{134 a, 134 b}$, G. Tong ${ }^{32 a}$, A. Tonoyan ${ }^{13}$, C. Topfel ${ }^{16}$, N.D. Topilin ${ }^{65}$, I. Torchiani ${ }^{29}$, E. Torrence ${ }^{114}$,

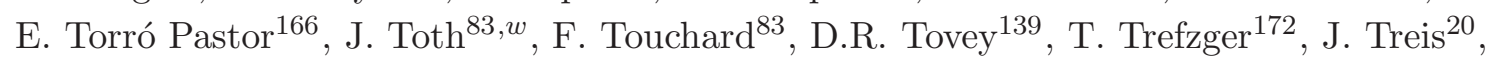
L. Tremblet ${ }^{29}$, A. Tricoli ${ }^{29}$, I.M. Trigger ${ }^{158 a}$, G. Trilling ${ }^{14}$, S. Trincaz-Duvoid ${ }^{78}$,

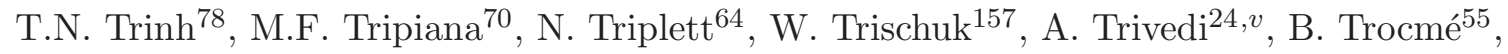
C. Troncon ${ }^{89 a}$, A. Trzupek ${ }^{38}$, C. Tsarouchas ${ }^{9}$, J.C-L. Tseng ${ }^{118}$, M. Tsiakiris ${ }^{105}$, P.V. Tsiareshka ${ }^{90}$, D. Tsionou ${ }^{139}$, G. Tsipolitis ${ }^{9}$, V. Tsiskaridze ${ }^{51}$, E.G. $_{\text {Tskhadadze }}{ }^{51}$, I.I. Tsukerman ${ }^{95}$, V. Tsulaia ${ }^{123}$, J.-W. Tsung ${ }^{20}$, S. Tsuno ${ }^{66}$, D. Tsybychev ${ }^{147}$, J.M. Tuggle ${ }^{30}$, M. Turala ${ }^{38}$, D. Turecek ${ }^{127}$, I. Turk Cakir ${ }^{3 e}$, E. Turlay ${ }^{105}$, P.M. Tuts ${ }^{34}$, M.S. Twomey ${ }^{138}$, M. Tylmad ${ }^{145 a, 145 b}$, M. Tyndel ${ }^{129}$, D. Typaldos ${ }^{17}$, H. Tyrvainen ${ }^{29}$,

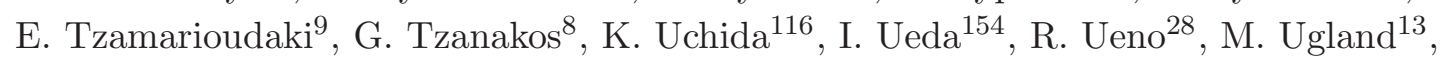
M. Uhlenbrock ${ }^{20}$, M. Uhrmacher ${ }^{54}$, F. Ukegawa ${ }^{159}$, G. Unal ${ }^{29}$, D.G. Underwood ${ }^{5}$, A. Undrus ${ }^{24}$, G. Unel ${ }^{162}$, Y. Unno ${ }^{66}$, D. Urbaniec ${ }^{34}$, E. Urkovsky ${ }^{152}$, P. Urquijo ${ }^{49, a b}$, P. Urrejola ${ }^{31 a}$, G. Usai ${ }^{7}$, M. Uslenghi ${ }^{119 a, 119 b}$, L. Vacavant ${ }^{83}$, V. Vacek ${ }^{127}$, B. Vachon ${ }^{85}$, S. Vahsen ${ }^{14}$, C. Valderanis ${ }^{99}$, J. Valenta ${ }^{125}$, P. Valente ${ }^{132 a}$, S. Valentinetti ${ }^{19 a, 19 b}$, S. Valkar ${ }^{126}$, E. Valladolid Gallego ${ }^{166}$, S. Vallecorsa ${ }^{151}$, J.A. Valls Ferrer ${ }^{166}$, R. Van Berg ${ }^{120}$, H. van der Graaf ${ }^{105}$, E. van der Kraaij ${ }^{105}$, E. van der Poel ${ }^{105}$, D. van der Ster ${ }^{29}$, B. Van Eijk ${ }^{105}$, N. van $\operatorname{Eldik}^{84}$, P. van Gemmeren ${ }^{5}$, Z. van Kesteren ${ }^{105}$, I. van Vulpen ${ }^{105}$, W. Vandelli ${ }^{29}$, G. Vandoni ${ }^{29}$, A. Vaniachine ${ }^{5}$, P. Vankov ${ }^{73}$,

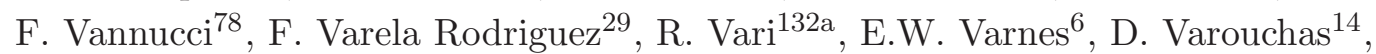

A. Vartapetian ${ }^{7}$, K.E. Varvell ${ }^{149}$, L. Vasilyeva ${ }^{94}$, V.I. Vassilakopoulos ${ }^{56}$, F. Vazeille ${ }^{33}$,

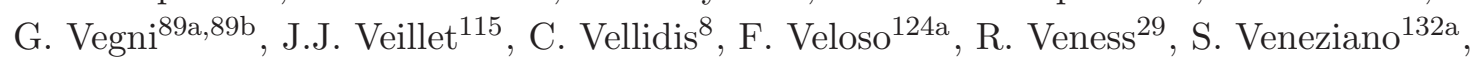
A. Ventura ${ }^{72 a, 72 b}$, D. Ventura ${ }^{138}$, S. Ventura ${ }^{47}$, M. Venturi ${ }^{48}$, N. Venturi ${ }^{16}$, V. Vercesi ${ }^{119 a}$, M. Verducci ${ }^{138}$, W. Verkerke ${ }^{105}$, J.C. Vermeulen ${ }^{105}$, L. Vertogardov ${ }^{118}$, 
M.C. Vetterli ${ }^{142, c}$, I. Vichou ${ }^{164}$, T. Vickey ${ }^{118}$, G.H.A. Viehhauser ${ }^{118}$, M. Villa ${ }^{19 a, 19 b}$, E.G. Villani ${ }^{129}$, M. Villaplana Perez ${ }^{166}$, E. Vilucchi ${ }^{47}$, M.G. Vincter ${ }^{28}$, E. Vinek ${ }^{29}$, V.B. Vinogradov ${ }^{65}$, M. Virchaux ${ }^{136, *}$, S. Viret ${ }^{33}$, J. Virzi ${ }^{14}$, A. Vitale ${ }^{19 a, 19 b}$, O. Vitells ${ }^{170}$, I. Vivarelli ${ }^{48}$, F. Vives Vaque ${ }^{11}$, S. Vlachos $^{9}$, M. Vlasak ${ }^{127}$, N. Vlasov ${ }^{20}$,

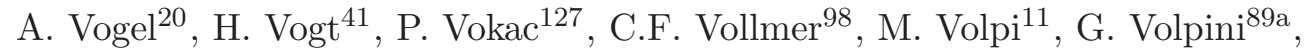
H. von der Schmitt ${ }^{99}$, J. von Loeben ${ }^{99}$, H. von Radziewski ${ }^{48}$, E. von Toerne ${ }^{20}$,

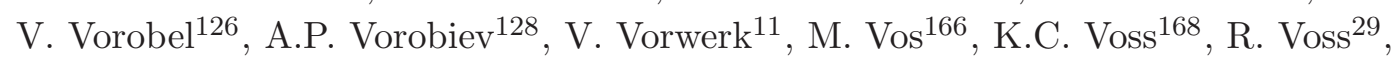
T.T. Voss ${ }^{173}$, J.H. Vossebeld ${ }^{73}$, A.S. Vovenko ${ }^{128}$, N. Vranjes ${ }^{12 a}$,

M. Vranjes Milosavljevic ${ }^{12 a}$, V. Vrba ${ }^{125}$, M. Vreeswijk ${ }^{105}$, T. Vu Anh ${ }^{81}$, B. Vuaridel ${ }^{49}$, D. Vudragovic ${ }^{12 a}$, R. Vuillermet ${ }^{29}$, I. Vukotic ${ }^{115}$, P. Wagner ${ }^{120}$, H. Wahlen ${ }^{173}$,

J. Walbersloh ${ }^{42}$, J. Walder ${ }^{71}$, R. Walker ${ }^{98}$, W. Walkowiak ${ }^{141}$, R. Wall ${ }^{174}$, S. Walsh $^{139}$, C. Wang ${ }^{44}$, H. Wang ${ }^{171}$, J. Wang ${ }^{55}$, J.C. Wang ${ }^{138}$, S.M. Wang ${ }^{150}$, A. Warburton ${ }^{85}$, C.P. Ward ${ }^{27}$, M. Warsinsky ${ }^{48}$, R. Wastie ${ }^{118}$, P.M. Watkins ${ }^{17}$, A.T. Watson ${ }^{17}$, M.F. Watson ${ }^{17}$, G. Watts ${ }^{138}$, S. Watts ${ }^{82}$, A.T. Waugh ${ }^{149}$, B.M. Waugh ${ }^{77}$, M. Webel ${ }^{48}$, G. Weber ${ }^{81}$, J. Weber ${ }^{42}$, M.D. Weber ${ }^{16}$, M. Weber ${ }^{129}$, M.S. Weber ${ }^{16}$, P. Weber ${ }^{54}$, A.R. Weidberg ${ }^{118}$, J. Weingarten ${ }^{54}$, C. Weiser ${ }^{48}$, H. Wellenstein ${ }^{22}$, H.P. Wellisch ${ }^{158 a}$, P.S. Wells ${ }^{29}$, M. Wen ${ }^{47}$, T. Wenaus ${ }^{24}$, S. Wendler ${ }^{123}$, Z. Weng ${ }^{150, a c}$, T. Wengler ${ }^{82}$, S. Wenig ${ }^{29}$, N. Wermes ${ }^{20}$, M. Werner ${ }^{48}$, P. Werner ${ }^{29}$, M. Werth ${ }^{162}$, U. Werthenbach ${ }^{141}$, M. Wessels ${ }^{58 a}$, K. Whalen ${ }^{28}$, S.J. Wheeler-Ellis ${ }^{162}$, S.P. Whitaker ${ }^{21}$, A. White ${ }^{7}$, M.J. White ${ }^{27}$, S. White ${ }^{24}$, S.R. Whitehead ${ }^{118}$, D. Whiteson ${ }^{162}$, D. Whittington ${ }^{61}$, F. Wicek ${ }^{115}$, D. Wicke ${ }^{81}$, F.J. Wickens ${ }^{129}$, W. Wiedenmann ${ }^{171}$, M. Wielers ${ }^{129}$, P. Wienemann ${ }^{20}$, M. Wiesmann ${ }^{99}$, C. Wiglesworth ${ }^{73}$, L.A.M. Wiik ${ }^{48}$, A. Wildauer ${ }^{166}$, M.A. Wildt ${ }^{41, m}$, I. Wilhelm ${ }^{126}$, H.G. Wilkens ${ }^{29}$, E. Williams ${ }^{34}$, H.H. Williams ${ }^{120}$, W. Willis ${ }^{34}$, S. Willocq ${ }^{84}$, J.A. Wilson ${ }^{17}$, M.G. Wilson ${ }^{143}$, A. Wilson ${ }^{87}$,

I. Wingerter-Seez ${ }^{4}$, F. Winklmeier ${ }^{29}$, M. Wittgen ${ }^{143}$, E. Woehrling ${ }^{17}$, M.W. Wolter ${ }^{38}$, H. Wolters ${ }^{124 a}$, B.K. Wosiek ${ }^{38}$, J. Wotschack ${ }^{29}$, M.J. Woudstra ${ }^{84}$, K. Wraight ${ }^{53}$, C. Wright ${ }^{53}$, D. Wright ${ }^{143}$, B. Wrona ${ }^{73}$, S.L. $\mathrm{Wu}^{171}, \mathrm{X} . \mathrm{Wu}^{49}$, J. Wuestenfeld ${ }^{42}$, E. Wulf ${ }^{34}$, R. Wunstorf ${ }^{42}$, B.M. Wynne ${ }^{45}$, L. Xaplanteris ${ }^{9}$, S. Xella ${ }^{35}$, S. Xie $^{48}$, Y. Xie $^{32 a}$,

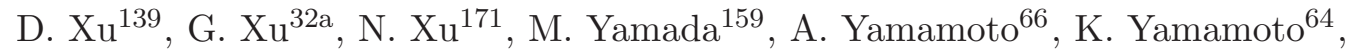
S. Yamamoto ${ }^{154}$, T. Yamamura ${ }^{154}$, J. Yamaoka ${ }^{44}$, T. Yamazaki ${ }^{154}$, Y. Yamazaki ${ }^{67}$,

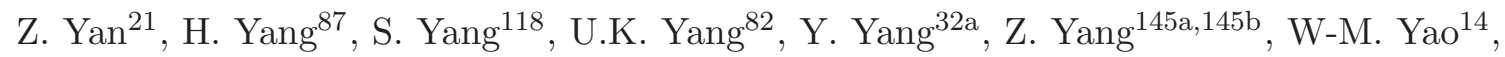

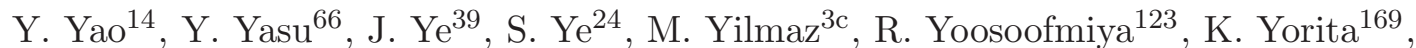

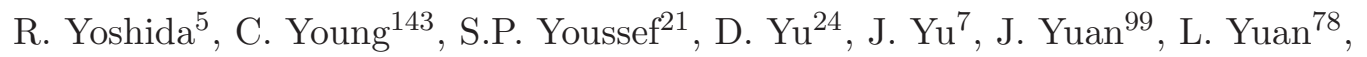
A. Yurkewicz ${ }^{147}$, V.G. Zaets ${ }^{128}$, R. Zaidan ${ }^{63}$, A.M. Zaitsev ${ }^{128}$, Z. Zajacova ${ }^{29}$, Yo.K. Zalite ${ }^{121}$, V. Zambrano ${ }^{47}$, L. Zanello132a,132b , P. Zarzhitsky ${ }^{39}$, A. Zaytsev ${ }^{107}$, M. Zdrazil ${ }^{14}$, C. Zeitnitz ${ }^{173}$, M. Zeller ${ }^{174}$, P.F. Zema ${ }^{29}$, A. Zemla ${ }^{38}$, C. Zendler ${ }^{20}$, A.V. Zenin ${ }^{128}$, O. Zenin ${ }^{128}$, T. Zenis ${ }^{144 a}$, Z. Zenonos ${ }^{122 a, 122 b}$, S. Zenz ${ }^{14}$, D. Zerwas ${ }^{115}$, G. Zevi della Porta ${ }^{57}$, Z. Zhan ${ }^{32 d}$, H. Zhang ${ }^{83}$, J. Zhang ${ }^{5}$, Q. Zhang ${ }^{5}$, X. Zhang ${ }^{32 d}$, L. Zhao ${ }^{108}$, T. Zhao ${ }^{138}$, Z. Zhao ${ }^{32 b}$, A. Zhemchugov ${ }^{65}$, S. Zheng ${ }^{32 a}$, J. Zhong ${ }^{150, a d}$, B. Zhou ${ }^{87}$, N. Zhou ${ }^{34}$, Y. Zhou ${ }^{150}$, C.G. Zhu ${ }^{32 \mathrm{~d}}$, H. Zhu ${ }^{41}$, Y. Zhu ${ }^{171}$, X. Zhuang ${ }^{98}$, V. Zhuravlov ${ }^{99}$, B. Zilka ${ }^{144 a}$, R. Zimmermann ${ }^{20}$, S. Zimmermann ${ }^{20}$, S. Zimmermann ${ }^{48}$, M. Ziolkowski ${ }^{141}$, R. Zitoun ${ }^{4}$, L. Živković ${ }^{34}$, V.V. Zmouchko ${ }^{128, *}$, G. Zobernig ${ }^{171}$, A. Zoccoli ${ }^{19 a, 19 b}$, Y. Zolnierowski ${ }^{4}$, A. Zsenei ${ }^{29}$, M. zur Nedden ${ }^{15}$, V. Zutshi ${ }^{106 a}$. 
${ }^{1}$ University at Albany, 1400 Washington Ave, Albany, NY 12222, United States of America

${ }^{2}$ University of Alberta, Department of Physics, Centre for Particle Physics, Edmonton, AB T6G 2G7, Canada

${ }^{3}$ Ankara University ${ }^{(a)}$, Faculty of Sciences, Department of Physics, TR 061000

Tandogan, Ankara; Dumlupinar University ${ }^{(b)}$, Faculty of Arts and Sciences, Department of Physics, Kutahya; Gazi University ${ }^{(c)}$, Faculty of Arts and Sciences, Department of Physics, 06500, Teknikokullar, Ankara; TOBB University of Economics and Technology ${ }^{(d)}$, Faculty of Arts and Sciences, Division of Physics, 06560, Sogutozu, Ankara; Turkish Atomic Energy Authority ${ }^{(e)}$, 06530, Lodumlu, Ankara, Turkey

${ }^{4}$ LAPP, Université de Savoie, CNRS/IN2P3, Annecy-le-Vieux, France

5 Argonne National Laboratory, High Energy Physics Division, 9700 S. Cass Avenue, Argonne IL 60439, United States of America

${ }^{6}$ University of Arizona, Department of Physics, Tucson, AZ 85721, United States of America

7 The University of Texas at Arlington, Department of Physics, Box 19059, Arlington, TX 76019, United States of America

${ }^{8}$ University of Athens, Nuclear \& Particle Physics, Department of Physics, Panepistimiopouli, Zografou, GR 15771 Athens, Greece

${ }^{9}$ National Technical University of Athens, Physics Department, 9-Iroon Polytechniou, GR 15780 Zografou, Greece

${ }^{10}$ Institute of Physics, Azerbaijan Academy of Sciences, H. Javid Avenue 33, AZ 143

Baku, Azerbaijan

${ }^{11}$ Institut de Física d'Altes Energies, IFAE, Edifici Cn, Universitat Autònoma de

Barcelona, ES - 08193 Bellaterra (Barcelona), Spain

${ }^{12}$ University of Belgrade ${ }^{(a)}$, Institute of Physics, P.O. Box 57, 11001 Belgrade; Vinca Institute of Nuclear Sciences ${ }^{(b)}$ Mihajla Petrovica Alasa 12-14, 11001 Belgrade, Serbia

${ }^{13}$ University of Bergen, Department for Physics and Technology, Allegaten 55, NO - 5007

Bergen, Norway

14 Lawrence Berkeley National Laboratory and University of California, Physics Division, MS50B-6227, 1 Cyclotron Road, Berkeley, CA 94720, United States of America

${ }^{15}$ Humboldt University, Institute of Physics, Berlin, Newtonstr. 15, D-12489 Berlin,

Germany

${ }^{16}$ University of Bern, Albert Einstein Center for Fundamental Physics, Laboratory for High Energy Physics, Sidlerstrasse 5, CH - 3012 Bern, Switzerland

17 University of Birmingham, School of Physics and Astronomy, Edgbaston, Birmingham B15 2TT, United Kingdom

18 Bogazici University ${ }^{(a)}$, Faculty of Sciences, Department of Physics, TR - 80815

Bebek-Istanbul; Dogus University ${ }^{(b)}$, Faculty of Arts and Sciences, Department of Physics, 34722, Kadikoy, Istanbul; ${ }^{(c)}$ Gaziantep University, Faculty of Engineering, Department of Physics Engineering, 27310, Sehitkamil, Gaziantep, Turkey; Istanbul Technical University ${ }^{(d)}$, Faculty of Arts and Sciences, Department of Physics, 34469, Maslak, Istanbul, Turkey 
19 INFN Sezione di Bologna ${ }^{(a)}$; Università di Bologna, Dipartimento di Fisica ${ }^{(b)}$, viale C. Berti Pichat, 6/2, IT - 40127 Bologna, Italy

${ }^{20}$ University of Bonn, Physikalisches Institut, Nussallee 12, D - 53115 Bonn, Germany

${ }^{21}$ Boston University, Department of Physics, 590 Commonwealth Avenue, Boston, MA 02215, United States of America

${ }^{22}$ Brandeis University, Department of Physics, MS057, 415 South Street, Waltham, MA 02454, United States of America

${ }^{23}$ Universidade Federal do Rio De Janeiro, COPPE/EE/IF ${ }^{(a)}$, Caixa Postal 68528, Ilha do Fundao, BR - 21945-970 Rio de Janeiro; ${ }^{(b)}$ Universidade de Sao Paulo, Instituto de Fisica, R.do Matao Trav. R.187, Sao Paulo - SP, 05508 - 900, Brazil

${ }^{24}$ Brookhaven National Laboratory, Physics Department, Bldg. 510A, Upton, NY 11973, United States of America

${ }^{25}$ National Institute of Physics and Nuclear Engineering ${ }^{(a)}$, Bucharest-Magurele, Str. Atomistilor 407, P.O. Box MG-6, R-077125, Romania; University Politehnica Bucharest $^{(b)}$, Rectorat - AN 001, 313 Splaiul Independentei, sector 6, 060042 Bucuresti; West University ${ }^{(c)}$ in Timisoara, Bd. Vasile Parvan 4, Timisoara, Romania ${ }^{26}$ Universidad de Buenos Aires, FCEyN, Dto. Fisica, Pab I - C. Universitaria, 1428 Buenos Aires, Argentina

${ }^{27}$ University of Cambridge, Cavendish Laboratory, J J Thomson Avenue, Cambridge CB3 0HE, United Kingdom

${ }^{28}$ Carleton University, Department of Physics, 1125 Colonel By Drive, Ottawa ON K1S 5B6, Canada

${ }^{29}$ CERN, CH - 1211 Geneva 23, Switzerland

${ }^{30}$ University of Chicago, Enrico Fermi Institute, 5640 S. Ellis Avenue, Chicago, IL 60637, United States of America

${ }^{31}$ Pontificia Universidad Católica de Chile, Facultad de Fisica, Departamento de Fisica $^{(a)}$, Avda. Vicuna Mackenna 4860, San Joaquin, Santiago; Universidad Técnica Federico Santa María, Departamento de Física ${ }^{(b)}$, Avda. Espãna 1680, Casilla 110-V, Valparaíso, Chile

${ }^{32}$ Institute of High Energy Physics, Chinese Academy of Sciences ${ }^{(a)}$, P.O. Box 918, 19 Yuquan Road, Shijing Shan District, CN - Beijing 100049; University of Science \& Technology of China (USTC), Department of Modern Physics ${ }^{(b)}$, Hefei, CN - Anhui 230026; Nanjing University, Department of Physics ${ }^{(c)}$, 22 Hankou Road, Nanjing, 210093; Shandong University, High Energy Physics Group ${ }^{(d)}$, Jinan, CN - Shandong 250100, China

${ }^{33}$ Laboratoire de Physique Corpusculaire, Clermont Université, Université Blaise Pascal, CNRS/IN2P3, FR - 63177 Aubiere Cedex, France

${ }^{34}$ Columbia University, Nevis Laboratory, 136 So. Broadway, Irvington, NY 10533, United States of America

35 University of Copenhagen, Niels Bohr Institute, Blegdamsvej 17, DK - 2100 Kobenhavn 0, Denmark

${ }^{36}$ INFN Gruppo Collegato di Cosenza ${ }^{(a)}$; Università della Calabria, Dipartimento di Fisica $^{(b)}$, IT-87036 Arcavacata di Rende, Italy 
37 Faculty of Physics and Applied Computer Science of the AGH-University of Science and Technology, (FPACS, AGH-UST), al. Mickiewicza 30, PL-30059 Cracow, Poland

38 The Henryk Niewodniczanski Institute of Nuclear Physics, Polish Academy of Sciences, ul. Radzikowskiego 152, PL - 31342 Krakow, Poland

39 Southern Methodist University, Physics Department, 106 Fondren Science Building, Dallas, TX 75275-0175, United States of America

${ }^{40}$ University of Texas at Dallas, 800 West Campbell Road, Richardson, TX 75080-3021, United States of America

41 DESY, Notkestr. 85, D-22603 Hamburg and Platanenallee 6, D-15738 Zeuthen,

Germany

42 TU Dortmund, Experimentelle Physik IV, DE - 44221 Dortmund, Germany

43 Technical University Dresden, Institut für Kern- und Teilchenphysik, Zellescher Weg 19, D-01069 Dresden, Germany

${ }^{44}$ Duke University, Department of Physics, Durham, NC 27708, United States of America

${ }^{45}$ University of Edinburgh, School of Physics \& Astronomy, James Clerk Maxwell Building, The Kings Buildings, Mayfield Road, Edinburgh EH9 3JZ, United Kingdom

${ }^{46}$ Fachhochschule Wiener Neustadt; Johannes Gutenbergstrasse 3 AT - 2700 Wiener Neustadt, Austria

${ }^{47}$ INFN Laboratori Nazionali di Frascati, via Enrico Fermi 40, IT-00044 Frascati, Italy

48 Albert-Ludwigs-Universität, Fakultät für Mathematik und Physik, Hermann-Herder Str. 3, D - 79104 Freiburg i.Br., Germany

${ }^{49}$ Université de Genève, section de Physique, 24 rue Ernest Ansermet, CH - 1211 Geneve 4, Switzerland

${ }^{50}$ INFN Sezione di Genova ${ }^{(a)}$; Università di Genova, Dipartimento di Fisica ${ }^{(b)}$, via Dodecaneso 33, IT - 16146 Genova, Italy

${ }^{51}$ Institute of Physics of the Georgian Academy of Sciences, 6 Tamarashvili St., GE 380077 Tbilisi; Tbilisi State University, HEP Institute, University St. 9, GE - 380086 Tbilisi, Georgia

52 Justus-Liebig-Universität Giessen, II Physikalisches Institut, Heinrich-Buff Ring 16, D-35392 Giessen, Germany

${ }^{53}$ University of Glasgow, Department of Physics and Astronomy, Glasgow G12 8QQ, United Kingdom

${ }^{54}$ Georg-August-Universität, II. Physikalisches Institut, Friedrich-Hund Platz 1, D-37077 Göttingen, Germany

${ }^{55}$ Laboratoire de Physique Subatomique et de Cosmologie, CNRS/IN2P3, Université Joseph Fourier, INPG, 53 avenue des Martyrs, FR - 38026 Grenoble Cedex, France ${ }^{56}$ Hampton University, Department of Physics, Hampton, VA 23668, United States of America

${ }^{57}$ Harvard University, Laboratory for Particle Physics and Cosmology, 18 Hammond Street, Cambridge, MA 02138, United States of America

58 Ruprecht-Karls-Universität Heidelberg: Kirchhoff-Institut für Physik ${ }^{(a)}, \mathrm{Im}$ Neuenheimer Feld 227, D-69120 Heidelberg; Physikalisches Institut ${ }^{(b)}$, Philosophenweg 12, D-69120 Heidelberg; ZITI Ruprecht-Karls-University Heidelberg ${ }^{(c)}$, Lehrstuhl für 
Informatik V, B6, 23-29, DE - 68131 Mannheim, Germany

${ }^{59}$ Hiroshima University, Faculty of Science, 1-3-1 Kagamiyama, Higashihiroshima-shi, JP - Hiroshima 739-8526, Japan

${ }^{60}$ Hiroshima Institute of Technology, Faculty of Applied Information Science, 2-1-1 Miyake Saeki-ku, Hiroshima-shi, JP - Hiroshima 731-5193, Japan

${ }^{61}$ Indiana University, Department of Physics, Swain Hall West 117, Bloomington, IN 47405-7105, United States of America

${ }^{62}$ Institut für Astro- und Teilchenphysik, Technikerstrasse 25, A - 6020 Innsbruck, Austria

${ }^{63}$ University of Iowa, 203 Van Allen Hall, Iowa City, IA 52242-1479, United States of America

${ }^{64}$ Iowa State University, Department of Physics and Astronomy, Ames High Energy Physics Group, Ames, IA 50011-3160, United States of America

65 Joint Institute for Nuclear Research, JINR Dubna, RU - 141980 Moscow Region, Russia

${ }^{66}$ KEK, High Energy Accelerator Research Organization, 1-1 Oho, Tsukuba-shi, Ibaraki-ken 305-0801, Japan

${ }^{67}$ Kobe University, Graduate School of Science, 1-1 Rokkodai-cho, Nada-ku, JP Kobe 657-8501, Japan

${ }^{68}$ Kyoto University, Faculty of Science, Oiwake-cho, Kitashirakawa, Sakyou-ku, Kyoto-shi, JP - Kyoto 606-8502, Japan

${ }^{69}$ Kyoto University of Education, 1 Fukakusa, Fujimori, fushimi-ku, Kyoto-shi, JP Kyoto 612-8522, Japan

${ }^{70}$ Universidad Nacional de La Plata, FCE, Departamento de Física, IFLP (CONICET-UNLP), C.C. 67, 1900 La Plata, Argentina

${ }^{71}$ Lancaster University, Physics Department, Lancaster LA1 4YB, United Kingdom

${ }^{72}$ INFN Sezione di Lecce ${ }^{(a)}$; Università del Salento, Dipartimento di Fisica ${ }^{(b)}$ Via Arnesano IT - 73100 Lecce, Italy

${ }^{73}$ University of Liverpool, Oliver Lodge Laboratory, P.O. Box 147, Oxford Street, Liverpool L69 3BX, United Kingdom

74 Jožef Stefan Institute and University of Ljubljana, Department of Physics, SI-1000 Ljubljana, Slovenia

${ }^{75}$ Queen Mary University of London, Department of Physics, Mile End Road, London E1 4NS, United Kingdom

${ }^{76}$ Royal Holloway, University of London, Department of Physics, Egham Hill, Egham, Surrey TW20 0EX, United Kingdom

77 University College London, Department of Physics and Astronomy, Gower Street, London WC1E 6BT, United Kingdom

${ }^{78}$ Laboratoire de Physique Nucléaire et de Hautes Energies, Université Pierre et Marie Curie (Paris 6), Université Denis Diderot (Paris-7), CNRS/IN2P3, Tour 33, 4 place Jussieu, FR - 75252 Paris Cedex 05, France

${ }^{79}$ Lunds universitet, Naturvetenskapliga fakulteten, Fysiska institutionen, Box 118, SE 22100 Lund, Sweden 
${ }^{80}$ Universidad Autonoma de Madrid, Facultad de Ciencias, Departamento de Fisica Teorica, ES - 28049 Madrid, Spain

${ }^{81}$ Universität Mainz, Institut für Physik, Staudinger Weg 7, DE - 55099 Mainz, Germany

${ }^{82}$ University of Manchester, School of Physics and Astronomy, Manchester M13 9PL, United Kingdom

${ }^{83}$ CPPM, Aix-Marseille Université, CNRS/IN2P3, Marseille, France

${ }^{84}$ University of Massachusetts, Department of Physics, 710 North Pleasant Street, Amherst, MA 01003, United States of America

85 McGill University, High Energy Physics Group, 3600 University Street, Montreal, Quebec H3A 2T8, Canada

${ }^{86}$ University of Melbourne, School of Physics, AU - Parkville, Victoria 3010, Australia

87 The University of Michigan, Department of Physics, 2477 Randall Laboratory, 500

East University, Ann Arbor, MI 48109-1120, United States of America

${ }^{88}$ Michigan State University, Department of Physics and Astronomy, High Energy

Physics Group, East Lansing, MI 48824-2320, United States of America

${ }^{89}$ INFN Sezione di Milano $^{(a)}$; Università di Milano, Dipartimento di Fisica ${ }^{(b)}$, via Celoria 16, IT - 20133 Milano, Italy

${ }^{90}$ B.I. Stepanov Institute of Physics, National Academy of Sciences of Belarus,

Independence Avenue 68, Minsk 220072, Republic of Belarus

${ }^{91}$ National Scientific \& Educational Centre for Particle \& High Energy Physics, NC

PHEP BSU, M. Bogdanovich St. 153, Minsk 220040, Republic of Belarus

92 Massachusetts Institute of Technology, Department of Physics, Room 24-516,

Cambridge, MA 02139, United States of America

${ }^{93}$ University of Montreal, Group of Particle Physics, C.P. 6128, Succursale Centre-Ville, Montreal, Quebec, H3C 3J7, Canada

${ }^{94}$ P.N. Lebedev Institute of Physics, Academy of Sciences, Leninsky pr. 53, RU - 117924 Moscow, Russia

${ }^{95}$ Institute for Theoretical and Experimental Physics (ITEP), B. Cheremushkinskaya ul. 25, RU 117218 Moscow, Russia

${ }^{96}$ Moscow Engineering \& Physics Institute (MEPhI), Kashirskoe Shosse 31, RU - 115409 Moscow, Russia

${ }^{97}$ Lomonosov Moscow State University Skobeltsyn Institute of Nuclear Physics (MSU SINP), 1(2), Leninskie gory, GSP-1, Moscow 119991 Russian Federation, Russia

${ }^{98}$ Ludwig-Maximilians-Universität München, Fakultät für Physik, Am Coulombwall 1, DE - 85748 Garching, Germany

${ }^{99}$ Max-Planck-Institut für Physik, (Werner-Heisenberg-Institut), Föhringer Ring 6, 80805 München, Germany

100 Nagasaki Institute of Applied Science, 536 Aba-machi, JP Nagasaki 851-0193, Japan

101 Nagoya University, Graduate School of Science, Furo-Cho, Chikusa-ku, Nagoya, 464-8602, Japan

102 INFN Sezione di Napoli ${ }^{(a)}$; Università di Napoli, Dipartimento di Scienze Fisiche ${ }^{(b)}$, Complesso Universitario di Monte Sant'Angelo, via Cinthia, IT - 80126 Napoli, Italy 103 University of New Mexico, Department of Physics and Astronomy, MSC07 4220, 
Albuquerque, NM 87131 USA, United States of America

104 Radboud University Nijmegen/NIKHEF, Department of Experimental High Energy Physics, Heyendaalseweg 135, NL-6525 AJ, Nijmegen, Netherlands

105 Nikhef National Institute for Subatomic Physics, and University of Amsterdam, Science Park 105, 1098 XG Amsterdam, Netherlands

$106{ }^{(a)}$ DeKalb, Illinois 60115, United States of America

107 Budker Institute of Nuclear Physics (BINP), RU - Novosibirsk 630 090, Russia

108 New York University, Department of Physics, 4 Washington Place, New York NY 10003, USA, United States of America

109 Ohio State University, 191 West Woodruff Ave, Columbus, OH 43210-1117, United

States of America

110 Okayama University, Faculty of Science, Tsushimanaka 3-1-1, Okayama 700-8530, Japan

${ }^{111}$ University of Oklahoma, Homer L. Dodge Department of Physics and Astronomy, 440

West Brooks, Room 100, Norman, OK 73019-0225, United States of America

112 Oklahoma State University, Department of Physics, 145 Physical Sciences Building, Stillwater, OK 74078-3072, United States of America

113 Palacký University, 17.listopadu 50a, 77207 Olomouc, Czech Republic

${ }^{114}$ University of Oregon, Center for High Energy Physics, Eugene, OR 97403-1274,

United States of America

115 LAL, Univ. Paris-Sud, IN2P3/CNRS, Orsay, France

116 Osaka University, Graduate School of Science, Machikaneyama-machi 1-1, Toyonaka, Osaka 560-0043, Japan

117 University of Oslo, Department of Physics, P.O. Box 1048, Blindern, NO - 0316 Oslo

3, Norway

118 Oxford University, Department of Physics, Denys Wilkinson Building, Keble Road, Oxford OX1 3RH, United Kingdom

119 INFN Sezione di Pavia ${ }^{(a)}$; Università di Pavia, Dipartimento di Fisica Nucleare e Teorica $^{(b)}$, Via Bassi 6, IT-27100 Pavia, Italy

${ }^{120}$ University of Pennsylvania, Department of Physics, High Energy Physics Group, 209

S. 33rd Street, Philadelphia, PA 19104, United States of America

121 Petersburg Nuclear Physics Institute, RU - 188300 Gatchina, Russia

122 INFN Sezione di Pisa ${ }^{(a)}$; Università di Pisa, Dipartimento di Fisica E. Fermi ${ }^{(b)}$, Largo

B. Pontecorvo 3, IT - 56127 Pisa, Italy

${ }^{123}$ University of Pittsburgh, Department of Physics and Astronomy, 3941 O'Hara Street, Pittsburgh, PA 15260, United States of America

${ }^{124}$ Laboratorio de Instrumentacao e Fisica Experimental de Particulas - $\operatorname{LIP}^{(a)}$, Avenida

Elias Garcia 14-1, PT - 1000-149 Lisboa, Portugal; Universidad de Granada,

Departamento de Fisica Teorica y del Cosmos and CAFPE ${ }^{(b)}$, E-18071 Granada, Spain

${ }^{125}$ Institute of Physics, Academy of Sciences of the Czech Republic, Na Slovance 2, CZ -

18221 Praha 8, Czech Republic

126 Charles University in Prague, Faculty of Mathematics and Physics, Institute of

Particle and Nuclear Physics, V Holesovickach 2, CZ - 18000 Praha 8, Czech Republic 
127 Czech Technical University in Prague, Zikova 4, CZ - 16635 Praha 6, Czech Republic 128 State Research Center Institute for High Energy Physics, Moscow Region, 142281, Protvino, Pobeda street, 1, Russia

129 Rutherford Appleton Laboratory, Science and Technology Facilities Council, Harwell Science and Innovation Campus, Didcot OX11 0QX, United Kingdom

130 University of Regina, Physics Department, Canada

131 Ritsumeikan University, Noji Higashi 1 chome 1-1, JP - Kusatsu, Shiga 525-8577, Japan

132 INFN Sezione di Roma $\mathrm{I}^{(a)}$; Università La Sapienza, Dipartimento di Fisica ${ }^{(b)}$, Piazzale A. Moro 2, IT- 00185 Roma, Italy

133 INFN Sezione di Roma Tor Vergata ${ }^{(a)}$; Università di Roma Tor Vergata, Dipartimento di Fisica $^{(b)}$, via della Ricerca Scientifica, IT-00133 Roma, Italy ${ }^{134}$ INFN Sezione di Roma Tre ${ }^{(a)}$; Università Roma Tre, Dipartimento di Fisica ${ }^{(b)}$, via della Vasca Navale 84, IT-00146 Roma, Italy

135 Réseau Universitaire de Physique des Hautes Energies (RUPHE): Université Hassan II, Faculté des Sciences Ain Chock $^{(a)}$, B.P. 5366, MA - Casablanca; Centre National de l'Energie des Sciences Techniques Nucleaires (CNESTEN) ${ }^{(b)}$, B.P. 1382 R.P. 10001 Rabat 10001; Université Mohamed Premier ${ }^{(c)}$, LPTPM, Faculté des Sciences, B.P.717. Bd.

Mohamed VI, 60000, Oujda ; Université Mohammed V, Faculté des Sciences ${ }^{(d)} 4$ Avenue Ibn Battouta, BP 1014 RP, 10000 Rabat, Morocco

136 CEA, DSM/IRFU, Centre d'Etudes de Saclay, FR - 91191 Gif-sur-Yvette, France

137 University of California Santa Cruz, Santa Cruz Institute for Particle Physics (SCIPP), Santa Cruz, CA 95064, United States of America

138 University of Washington, Seattle, Department of Physics, Box 351560, Seattle, WA 98195-1560, United States of America

139 University of Sheffield, Department of Physics \& Astronomy, Hounsfield Road, Sheffield S3 7RH, United Kingdom

140 Shinshu University, Department of Physics, Faculty of Science, 3-1-1 Asahi, Matsumoto-shi, JP - Nagano 390-8621, Japan

141 Universität Siegen, Fachbereich Physik, D 57068 Siegen, Germany

142 Simon Fraser University, Department of Physics, 8888 University Drive, CA Burnaby, BC V5A 1S6, Canada

143 SLAC National Accelerator Laboratory, Stanford, California 94309, United States of America

144 Comenius University, Faculty of Mathematics, Physics \& Informatics ${ }^{(a)}$, Mlynska dolina F2, SK - 84248 Bratislava; Institute of Experimental Physics of the Slovak Academy of Sciences, Dept. of Subnuclear Physics ${ }^{(b)}$, Watsonova 47, SK - 04353 Kosice, Slovak Republic

145 Stockholm University: Department of Physics ${ }^{(a)}$; The Oskar Klein Centre ${ }^{(b)}$, AlbaNova, SE - 10691 Stockholm, Sweden

146 Royal Institute of Technology (KTH), Physics Department, SE - 10691 Stockholm, Sweden

147 Stony Brook University, Department of Physics and Astronomy, Nicolls Road, Stony 
Brook, NY 11794-3800, United States of America

148 University of Sussex, Department of Physics and Astronomy Pevensey 2 Building,

Falmer, Brighton BN1 9QH, United Kingdom

149 University of Sydney, School of Physics, AU - Sydney NSW 2006, Australia

150 Insitute of Physics, Academia Sinica, TW - Taipei 11529, Taiwan

151 Technion, Israel Inst. of Technology, Department of Physics, Technion City, IL - Haifa 32000, Israel

152 Tel Aviv University, Raymond and Beverly Sackler School of Physics and Astronomy, Ramat Aviv, IL - Tel Aviv 69978, Israel

153 Aristotle University of Thessaloniki, Faculty of Science, Department of Physics,

Division of Nuclear \& Particle Physics, University Campus, GR - 54124, Thessaloniki,

Greece

154 The University of Tokyo, International Center for Elementary Particle Physics and

Department of Physics, 7-3-1 Hongo, Bunkyo-ku, JP - Tokyo 113-0033, Japan

155 Tokyo Metropolitan University, Graduate School of Science and Technology, 1-1

Minami-Osawa, Hachioji, Tokyo 192-0397, Japan

156 Tokyo Institute of Technology, 2-12-1-H-34 O-Okayama, Meguro, Tokyo 152-8551,

Japan

157 University of Toronto, Department of Physics, 60 Saint George Street, Toronto M5S

1A7, Ontario, Canada

158 TRIUMF $^{(a)}, 4004$ Wesbrook Mall, Vancouver, B.C. V6T 2A3; ${ }^{(b)}$ York University,

Department of Physics and Astronomy, 4700 Keele St., Toronto, Ontario, M3J 1P3,

Canada

159 University of Tsukuba, Institute of Pure and Applied Sciences, 1-1-1 Tennoudai,

Tsukuba-shi, JP - Ibaraki 305-8571, Japan

160 Tufts University, Science \& Technology Center, 4 Colby Street, Medford, MA 02155,

United States of America

161 Universidad Antonio Narino, Centro de Investigaciones, Cra 3 Este No.47A-15,

Bogota, Colombia

162 University of California, Irvine, Department of Physics \& Astronomy, CA 92697-4575,

United States of America

${ }^{163}$ INFN Gruppo Collegato di Udine ${ }^{(a)} ; \operatorname{ICTP}^{(b)}$, Strada Costiera 11, IT-34014, Trieste;

Università di Udine, Dipartimento di Fisica ${ }^{(c)}$, via delle Scienze 208, IT - 33100 Udine,

Italy

164 University of Illinois, Department of Physics, 1110 West Green Street, Urbana, Illinois 61801, United States of America

165 University of Uppsala, Department of Physics and Astronomy, P.O. Box 516, SE -751

20 Uppsala, Sweden

${ }^{166}$ Instituto de Física Corpuscular (IFIC) Centro Mixto UVEG-CSIC, Apdo. 22085

ES-46071 Valencia, Dept. Física At. Mol. y Nuclear; Univ. of Valencia, and Instituto de Microelectrónica de Barcelona (IMB-CNM-CSIC) 08193 Bellaterra Barcelona, Spain

167 University of British Columbia, Department of Physics, 6224 Agricultural Road, CA Vancouver, B.C. V6T 1Z1, Canada 
168 University of Victoria, Department of Physics and Astronomy, P.O. Box 3055, Victoria B.C., V8W 3P6, Canada

169 Waseda University, WISE, 3-4-1 Okubo, Shinjuku-ku, Tokyo, 169-8555, Japan

170 The Weizmann Institute of Science, Department of Particle Physics, P.O. Box 26, IL 76100 Rehovot, Israel

${ }^{171}$ University of Wisconsin, Department of Physics, 1150 University Avenue, WI 53706

Madison, Wisconsin, United States of America

172 Julius-Maximilians-University of Würzburg, Physikalisches Institute, Am Hubland, 97074 Würzburg, Germany

173 Bergische Universität, Fachbereich C, Physik, Postfach 100127, Gauss-Strasse 20, D42097 Wuppertal, Germany

174 Yale University, Department of Physics, PO Box 208121, New Haven CT, 06520-8121, United States of America

175 Yerevan Physics Institute, Alikhanian Brothers Street 2, AM - 375036 Yerevan, Armenia

176 ATLAS-Canada Tier-1 Data Centre, TRIUMF, 4004 Wesbrook Mall, Vancouver, BC, V6T 2A3, Canada

177 GridKA Tier-1 FZK, Forschungszentrum Karlsruhe GmbH, Steinbuch Centre for Computing (SCC), Hermann-von-Helmholtz-Platz 1, 76344 Eggenstein-Leopoldshafen, Germany

178 Port d'Informacio Cientifica (PIC), Universitat Autonoma de Barcelona (UAB), Edifici D, E-08193 Bellaterra, Spain

${ }^{179}$ Centre de Calcul CNRS/IN2P3, Domaine scientifique de la Doua, 27 bd du 11 Novembre 1918, 69622 Villeurbanne Cedex, France

180 INFN-CNAF, Viale Berti Pichat 6/2, 40127 Bologna, Italy

181 Nordic Data Grid Facility, NORDUnet A/S, Kastruplundgade 22, 1, DK-2770

Kastrup, Denmark

182 SARA Reken- en Netwerkdiensten, Science Park 121, 1098 XG Amsterdam,

Netherlands

183 Academia Sinica Grid Computing, Institute of Physics, Academia Sinica, No.128, section 2, Academia Rd., Nankang, Taipei, Taiwan 11529, Taiwan

${ }^{184}$ UK-T1-RAL Tier-1, Rutherford Appleton Laboratory, Science and Technology

Facilities Council, Harwell Science and Innovation Campus, Didcot OX11 0QX, United Kingdom

185 RHIC and ATLAS Computing Facility, Physics Department, Building 510, Brookhaven National Laboratory, Upton, New York 11973, United States of America ${ }^{a}$ Present address FermiLab, USA

${ }^{b}$ Also at CPPM, Marseille, France.

${ }^{c}$ Also at TRIUMF, 4004 Wesbrook Mall, Vancouver, B.C. V6T 2A3, Canada

${ }^{d}$ Also at Faculty of Physics and Applied Computer Science of the AGH-University of Science and Technology, (FPACS, AGH-UST), al. Mickiewicza 30, PL-30059 Cracow, Poland

${ }^{e}$ Now at Universita' dell'Insubria, Dipartimento di Fisica e Matematica 
${ }^{f}$ Also at Università di Napoli Parthenope, via A. Acton 38, IT - 80133 Napoli, Italy

$g$ Also at Institute of Particle Physics (IPP), Canada

${ }^{h}$ Louisiana Tech University, 305 Wisteria Street, P.O. Box 3178, Ruston, LA 71272, United States of America

${ }^{i}$ At Department of Physics, California State University, Fresno, 2345 E. San Ramon Avenue, Fresno, CA 93740-8031, United States of America

${ }^{j}$ Currently at Istituto Universitario di Studi Superiori IUSS, V.le Lungo Ticino Sforza 56, 27100 Pavia, Italy

${ }^{k}$ Also at California Institute of Technology, Physics Department, Pasadena, CA 91125, United States of America

${ }^{l}$ Also at University of Montreal, Canada

$m$ Also at Institut für Experimentalphysik, Universität Hamburg, Luruper Chaussee 149, 22761 Hamburg, Germany

${ }^{n}$ Now at Chonnam National University, Chonnam, Korea 500-757

o Also at Petersburg Nuclear Physics Institute, RU - 188300 Gatchina, Russia

${ }^{p}$ Also at School of Physics and Engineering, Sun Yat-sen University, China

${ }^{q}$ Also at School of Physics, Shandong University, Jinan, China

${ }^{r}$ Also at Rutherford Appleton Laboratory, Science and Technology Facilities Council, Harwell Science and Innovation Campus, Didcot OX11, United Kingdom

${ }^{s}$ Also at school of physics, Shandong University, Jinan

${ }^{t}$ Also at Rutherford Appleton Laboratory, Science and Technology Facilities Council, Harwell Science and Innovation Campus, Didcot OX11 0QX, United Kingdom

${ }^{u}$ Now at KEK

${ }^{v}$ University of South Carolina, Dept. of Physics and Astronomy, 700 S. Main St, Columbia, SC 29208, United States of America

${ }^{w}$ Also at KFKI Research Institute for Particle and Nuclear Physics, Budapest, Hungary

${ }^{x}$ Now at TRIUMF, Vancouver, Canada.

y Also at Institute of Physics, Jagiellonian University, Cracow, Poland

${ }^{z}$ University of Rochester, Rochester, NY 14627, USA

aa Also at School of Physics and Engineering, Sun Yat-sen University, Taiwan

$a b$ Transfer to LHCb 31.01.2010

${ }^{a c}$ also at school of physics and engineering, Sun Yat-sen University

ad Also at Dept of Physics, Nanjing University, China

* Deceased 